\title{
Refinement Types for TypeScript
}

\author{
Panagiotis Vekris \\ University of California, San Diego \\ pvekris@cs.ucsd.edu
}

\author{
Benjamin Cosman \\ University of California, San Diego \\ blcosman@cs.ucsd.edu
}

\author{
Ranjit Jhala \\ University of California, San Diego \\ jhala@cs.ucsd.edu
}

\begin{abstract}
We present Refined TypeScript (RSC), a lightweight refinement type system for TypeScript, that enables static verification of higher-order, imperative programs. We develop a formal core of RSC that delineates the interaction between refinement types and mutability. Next, we extend the core to account for the imperative and dynamic features of TypeScript. Finally, we evaluate RSC on a set of real world benchmarks, including parts of the Octane benchmarks, D3, Transducers, and the TypeScript compiler.
\end{abstract}

\section{Introduction}

Modern scripting languages - like JavaScript, Python, and Ruby - have popularized the use of higher-order constructs that were once solely in the functional realm. This trend towards abstraction and reuse poses two related problems for static analysis: modularity and extensibility. First, how should analysis precisely track the flow of values across higher-order functions and containers or modularly account for external code like closures or library calls? Second, how can analyses be easily extended to new, domain specific properties, ideally by developers, while they are designing and implementing the code? (As opposed to by experts who can at best develop custom analyses run ex post facto and are of little use during development.)

Refinement types hold the promise of a precise, modular and extensible analysis for programs with higher-order functions and containers. Here, basic types are decorated with refinement predicates that constrain the values inhabiting the type $[29,39]$. The extensibility and modularity offered by refinement types have enabled their use in a variety of applications in typed, functional languages, like ML [28, 39], Haskell [37], and $F^{\sharp}$ [33]. Unfortunately, attempts to apply refinement typing to scripts have proven to be impractical due to the interaction of the machinery that accounts for imperative updates and higher-order functions [5] (\$6).

In this paper, we introduce Refined TypeScript (RSC): a novel, lightweight refinement type system for TypeScript, a typed superset of JavaScript. Our design of RSC addresses three intertwined problems by carefully integrating and extending existing ideas from the literature. First, RSC accounts for mutation by using ideas from IGJ [41] to track which fields may be mutated, and to allow refinements to depend on immutable fields, and by using SSA-form to recover path and flow-sensitivity that is essential for analyzing real world applications. Second, RSC accounts for dynamic typing by using a recently proposed technique called twophase typing [38], where dynamic behaviors are specified via union and intersection types, and verified by reduction to refinement typing. Third, the above are carefully designed to permit refinement inference via the Liquid Types [28] framework to render refinement typing practical on real world programs. Concretely, we make the following contributions:

- We develop a core calculus that formalizes the interaction of mutability and refinements via declarative refinement type checking that we prove sound ( $\$ 3)$.

- We extend the core language to TypeScript by describing how we account for its various dynamic and imperative features; in particular we show how RSC accounts for type reflection via intersection types, encodes interface hierarchies via refinements, and crucially permits locally flow-sensitive reasoning via SSA translation (§4).

- We implement rsc, a refinement type-checker for TypeScript, and evaluate it on a suite of real world programs from the Octane benchmarks, Transducers, D3 and the TypeScript compiler. We show that RSC's refinement typing is modular enough to analyze higherorder functions, collections and external code, and extensible enough to verify a variety of properties from classic array-bounds checking to program specific invariants needed to ensure safe reflection: critical invariants that are well beyond the scope of existing techniques for imperative scripting languages $(\$ 5)$.

\section{Overview}

We begin with a high-level overview of refinement types in RSC, their applications ( $\$ 2.1)$, and how RSC handles imperative, higher-order constructs ( $\$ 2.2)$.

Types and Refinements A basic refinement type is a basic type, e.g. number, refined with a logical formula from an SMT decidable logic [24]. For example, the types:

\footnotetext{
type nat $\quad=\{v$ :number $\mid 0 \leq v\}$

type pos $\quad=\{v$ :number $\mid 0<v\}$
} 


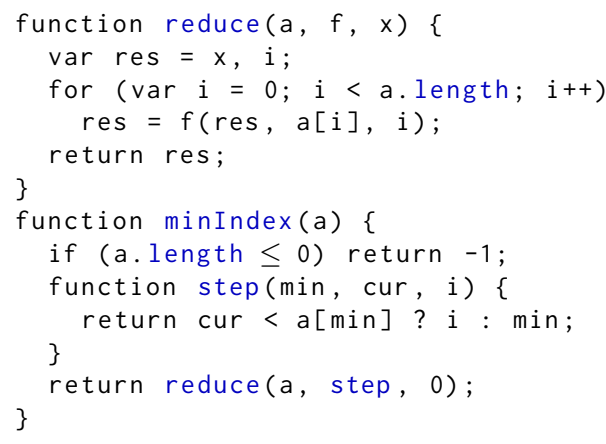

Figure 1: Computing the Min-Valued Index with reduce

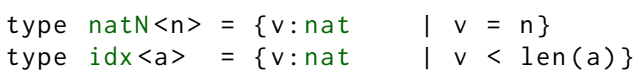

describe (the set of values corresponding to) non-negative numbers, positive numbers, numbers equal to some value $\mathrm{n}$, and valid indexes for an array a, respectively. Here, len is an uninterpreted function that describes the size of the array a. We write $t$ to abbreviate trivially refined types, i.e. $\{\mathrm{v}: \mathrm{t}$ | true $\} ;$ e.g. number abbreviates $\{\mathrm{v}$ : number | true $\}$.

Summaries Function Types $\left(x_{1}: \mathrm{T}_{1}, \ldots, x_{n}: \mathrm{T}_{n}\right) \Rightarrow \mathrm{T}$, where arguments are named $x_{i}$ and have types $\mathrm{T}_{i}$ and the output is a $T$, are used to specify the behavior of functions. In essence, the input types $\mathrm{T}_{i}$ specify the function's preconditions, and the output type $\mathrm{T}$ describes the postcondition. Each input type and the output type can refer to the arguments $x_{i}$, yielding precise function contracts. For example, $(x:$ nat $) \Rightarrow\{\nu$ :nat $\mid x<\nu\}$ is a function type that describes functions that require a non-negative input, and ensure that the output exceeds the input.

Higher-Order Summaries This approach generalizes directly to precise descriptions for higher-order functions. For example, reduce from Figure 1 can be specified as $T_{\text {reduce: }}$

$$
<A, B>(a: A[], \quad f:(B, A, \quad i d x<a>) \Rightarrow B, \quad x: B) \Rightarrow B
$$

This type is a precise summary for the higher-order behavior of reduce: it describes the relationship between the input array a, the step ("callback") function $f$, and the initial value of the accumulator, and stipulates that the output satisfies the same properties $\mathrm{B}$ as the input $\mathrm{x}$. Furthermore, it critically specifies that the callback $f$ is only invoked on valid indices for the array a being reduced.

\subsection{Applications}

Next, we show how refinement types let programmers specify and statically verify a variety of properties - array safety, reflection (value-based overloading), and down-casts - potential sources of runtime problems that cannot be prevented via existing techniques.

\subsubsection{Array Bounds}

Specification We specify safety by defining suitable refinement types for array creation and access. For example, we view read $a[i]$, write $a[i]=$ e and length access $a$. length as calls get $(a, i), \operatorname{set}(a, i, e)$ and length(a) where:

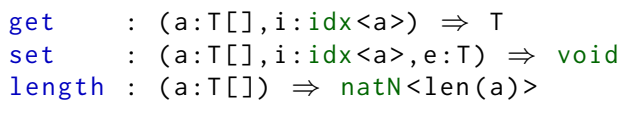

Verification Refinement typing ensures that the actual parameters supplied at each call to get and set are subtypes of the expected values specified in the signatures, and thus verifies that all accesses are safe. As an example, consider the function that returns the "head" element of an array:

function head $(\operatorname{arr}:$ NEArray $\langle T>)\{\operatorname{return}$ arr $[0] ;\}$

The input type requires that arr be non-empty:

type NEArray $\langle T\rangle=\{v: T[] \mid 0<\operatorname{len}(v)\}$

We convert $\operatorname{arr}[0]$ to get $(\operatorname{arr}, 0)$ which is checked under environment $\Gamma_{\text {head }}$ defined as arr : $\{\nu: \mathrm{T}[] \mid 0<\operatorname{len}(\nu)\}$ yielding the subtyping obligation:

$$
\Gamma_{\text {head }} \vdash\{\nu=0\} \sqsubseteq \operatorname{idx}\langle\operatorname{arr}\rangle
$$

which reduces to the logical verification condition (VC):

$$
0<\operatorname{len}(\operatorname{arr}) \Rightarrow(\nu=0 \Rightarrow 0 \leq \nu<\operatorname{len}(\operatorname{arr}))
$$

The VC is proved valid by an SMT solver [24], verifying subtyping, and hence, the array access' safety.

Path Sensitivity is obtained by adding branch conditions into the typing environment. Consider:

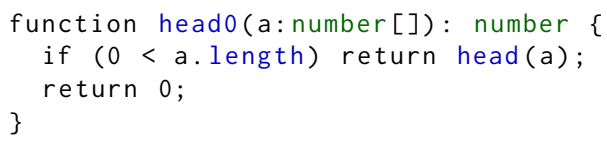

Recall that head should only be invoked with non-empty arrays. The call to head above occurs under $\Gamma_{\text {heado }}$ defined as: a : number[], $0<\operatorname{len}(\mathrm{a})$ i.e. which has the binder for the formal $a$, and the guard predicate established by the branch condition. Thus, the call to head yields the obligation:

$$
\Gamma_{\text {heado }} \vdash\{\nu=\mathrm{a}\} \sqsubseteq \text { NEArray }\langle\text { number }\rangle
$$

yielding the valid $\mathrm{VC}$ :

$$
0<\operatorname{len}(a) \Rightarrow(\nu=a \Rightarrow 0<\operatorname{len}(\nu))
$$

Polymorphic, Higher Order Functions Next, let us assume that reduce has the type $T_{\text {reduce }}$ described in (1), and see how to verify the array safety of minIndex (Figure 1). The challenge here is to precisely track which values can flow into min (used to index into a), which is tricky since those values are actually produced inside reduce.

Types make it easy to track such flows: we need only determine the instantiation of the polymorphic type variables of reduce at this call site inside minIndex. The type of the 
$f$ parameter in the instantiated type corresponds to a signature for the closure step which will let us verify the closure's implementation. Here, rsc automatically instantiates (by building complex logical predicates from simple terms that have been predefined in a prelude):

$$
\mathrm{A} \mapsto \text { number } \quad \mathrm{B} \mapsto \mathrm{idx}\langle\mathrm{a}\rangle
$$

Let us reassure ourselves that this instantiation is valid, by checking that step and 0 satisfy the instantiated type. If we substitute (2) into $T_{\text {reduce }}$ we obtain the following types for step and 0, i.e. reduce's second and third arguments:

$$
\text { step : }(i d x<a>\text {, number, } i d x<a>) \Rightarrow i d x<a>\quad 0: i d x<a>
$$

The initial value 0 is indeed a valid $i d x<a>$ thanks to the a. length check at the start of the function. To check step, assume that its inputs have the above types:

min: idx<a>, curr: number, i: idx<a>

The body is safe as the index $i$ is trivially a subtype of the required $i d x<a>$, and the output is one of min or $i$ and hence, of type $i d x<a>$ as required.

\subsubsection{Overloading}

Dynamic languages extensively use value-based overloading to simplify library interfaces. For example, a library may export:

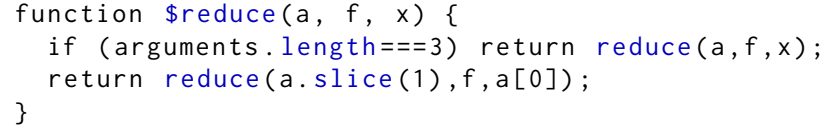

The function \$reduce has two distinct types depending on its parameters' values, rendering it impossible to statically type without path-sensitivity. Such overloading is ubiquitous: in more than $25 \%$ of libraries, more than $25 \%$ of the functions are value-overloaded [38].

Intersection Types Refinements let us statically verify valuebased overloading via an approach called Two-Phased Typing [38]. First, we specify overloading as an intersection type. For example, \$reduce gets the following signature, which is just the conjunction of the two overloaded behaviors:

$$
\begin{array}{ll}
<A, B>\left(a: A[]^{+},\right. & f:(A, A, \quad i d x<a>) \Rightarrow A) \Rightarrow A
\end{array} \begin{array}{ll}
/ / & 1 \\
<A, B>(a: A[], & f:(B, \quad A, \quad i d x<a>) \Rightarrow B, \quad x: B) \Rightarrow B / /
\end{array}
$$

The type $A[]^{+}$in the first conjunct indicates that the first argument needs to be a non-empty array, so that the call to slice and the access of $\mathrm{a}[0]$ both succeed.

Dead Code Assertions Second, we check each conjunct separately, replacing ill-typed terms in each context with assert(false). This requires the refinement type checker to prove that the corresponding expressions are dead code, as assert requires its argument to always be true:

assert : $(b:\{v:$ bool $\mid v=$ true $\}) \Rightarrow A$

To check \$reduce, we specialize it per overload context:

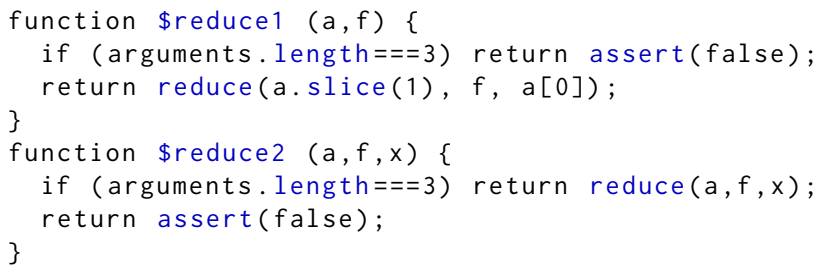

In each case, the "ill-typed" term (for the corresponding input context) is replaced with assert (false). Refinement typing easily verifies the asserts, as they respectively occur under the inconsistent environments:

$$
\begin{aligned}
& \Gamma_{1} \doteq \text { arguments: }\{\operatorname{len}(\nu)=2\}, \operatorname{len}(\text { arguments })=3 \\
& \Gamma_{2} \doteq \text { arguments: }\{\operatorname{len}(\nu)=3\}, \operatorname{len}(\text { arguments }) \neq 3
\end{aligned}
$$

which bind arguments to an array-like object corresponding to the arguments passed to that function, and include the branch condition under which the call to assert occurs.

\subsection{Analysis}

Next, we outline how rsc uses refinement types to analyze programs with closures, polymorphism, assignments, classes and mutation.

\subsubsection{Polymorphic Instantiation}

rsc uses the framework of Liquid Typing [28] to automatically synthesize the instantiations of (2). In a nutshell, rsc (a) creates templates for unknown refinement type instantiations, (b) performs type-checking over the templates to generate subtyping constraints over the templates that capture value-flow in the program, (c) solves the constraints via a fixpoint computation (abstract interpretation).

Step 1: Templates Recall that reduce has the polymorphic type $T_{\text {reduce }}$. At the call-site in minIndex, the type variables A, B are instantiated with the known base-type number. Thus, rsc creates fresh templates for the (instantiated) A, B:

$$
\mathrm{A} \mapsto\left\{\nu \text { :number } \mid \kappa_{A}\right\} \quad \mathrm{B} \mapsto\left\{\nu \text { :number } \mid \kappa_{B}\right\}
$$

where the refinement variables $\kappa_{A}$ and $\kappa_{B}$ represent the unknown refinements. We substitute the above in the signature for reduce to obtain a context-sensitive template:

$$
\left(\mathrm{a}: \kappa_{A}[],\left(\kappa_{B}, \kappa_{A}, \mathrm{idx}\langle\mathrm{a}\rangle\right) \Rightarrow \kappa_{B}, \kappa_{B}\right) \Rightarrow \kappa_{B}
$$

Step 2: Constraints Next, rsc generates subtyping constraints over the templates. Intuitively, the templates describe the sets of values that each static entity (e.g. variable) can evaluate to at runtime. The subtyping constraints capture the value-flow relationships e.g. at assignments, calls and returns, to ensure that the template solutions - and hence inferred refinements - soundly over-approximate the set of runtime values of each corresponding static entity.

We generate constraints by performing type checking over the templates. As a, 0, and step are passed in as arguments, we check that they respectively have the types $\kappa_{A}[]$, 
$\kappa_{B}$ and $\left(\kappa_{B}, \kappa_{A}\right.$, idx $\left.\langle\mathrm{a}\rangle\right) \Rightarrow \kappa_{B}$. Checking a and 0 yields the subtyping constraints:

$$
\Gamma \vdash \text { number }[] \sqsubseteq \kappa_{A}[] \quad \Gamma \vdash\{\nu=0\} \sqsubseteq \kappa_{B}
$$

where $\Gamma \doteq a$ :number [], $0<\operatorname{len}(a)$ from the else-guard that holds at the call to reduce. We check step by checking its body under the environment $\Gamma_{\text {step }}$ that binds the input parameters to their respective types:

$$
\Gamma_{\text {step }} \doteq \min : \kappa_{B}, \text { cur: } \kappa_{a}, i: \operatorname{idx}\langle a\rangle
$$

As min is used to index into the array a we get:

$$
\Gamma_{\text {step }} \vdash \kappa_{B} \sqsubseteq \operatorname{idx}\langle a\rangle
$$

As $i$ and min flow to the output type $\kappa_{B}$, we get:

$$
\Gamma_{\text {step }} \vdash \mathrm{idx}\langle\mathrm{a}\rangle \sqsubseteq \kappa_{B} \quad \Gamma_{\text {step }} \vdash \kappa_{B} \sqsubseteq \kappa_{B}
$$

Step 3: Fixpoint The above subtyping constraints over the $\kappa$ variables are reduced via the standard rules for co- and contra-variant subtyping, into Horn implications over the $\kappa s$. rsc solves the Horn implications via (predicate) abstract interpretation [28] to obtain the solution $\kappa_{A} \mapsto$ true and $\kappa_{B} \mapsto 0 \leq \nu<\operatorname{len}(a)$ which is exactly the instantiation in (2) that satisfies the subtyping constraints, and proves minIndex is array-safe.

\subsubsection{Assignments}

Next, let us see how the signature for reduce in Figure 1 is verified by rsc. Unlike in the functional setting, where refinements have previously been studied, here, we must deal with imperative features like assignments and for-loops.

SSA Transformation We solve this problem in three steps. First, we convert the code into SSA form, to introduce new binders at each assignment. Second, we generate fresh templates that represent the unknown types (i.e. set of values) for each $\phi$ variable. Third, we generate and solve the subtyping constraints to infer the types for the $\phi$-variables, and hence, the "loop-invariants" needed for verification.

Let us see how this process lets us verify reduce from Figure 1. First, we convert the body to SSA form (\$3.1)

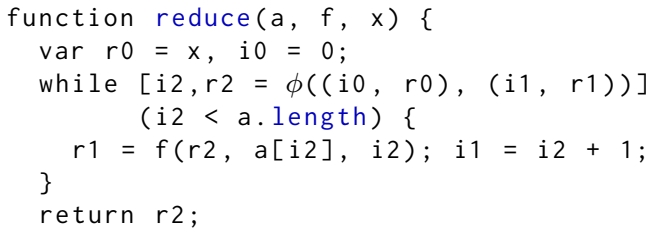

where i2 and $r 2$ are the $\phi$ variables for $i$ and $r$ respectively. Second, we generate templates for the $\phi$ variables:

$$
\text { i2: }\left\{\nu: \text { number } \mid \kappa_{i 2}\right\} \quad \mathrm{r} 2:\left\{\nu: \mathrm{B} \mid \kappa_{r 2}\right\}
$$

We need not generate templates for the SSA variables i0 , $r 0, i 1$ and $r 1$ as their types are those of the expressions they are assigned. Third, we generate subtyping constraints as before; the $\phi$ assignment generates additional constraints:

$$
\begin{array}{ll}
\Gamma_{0} \vdash\{\nu=\mathrm{i} 0\} \sqsubseteq \kappa_{i 2} & \Gamma_{1} \vdash\{\nu=\mathrm{i} 1\} \sqsubseteq \kappa_{i 2} \\
\Gamma_{0} \vdash\{\nu=\mathrm{r} 0\} \sqsubseteq \kappa_{r 2} & \Gamma_{1} \vdash\{\nu=\mathrm{r} 1\} \sqsubseteq \kappa_{r 2}
\end{array}
$$

where $\Gamma_{0}$ is the environment at the "exit" of the basic blocks where $\mathrm{i} 0, \mathrm{r} 0$ are defined:

$$
\left.\Gamma_{0} \doteq \mathrm{a}: \text { number[ }\right], \mathrm{x}: \mathrm{B}, \mathrm{i} 0: \operatorname{natN}\langle 0\rangle, \mathrm{r} 0:\{\nu: \mathrm{B} \mid \nu=\mathrm{x}\}
$$

Similarly, the environment $\Gamma_{1}$ includes bindings for variables $i 1$ and $r 1$. In addition, code executing the loop body has passed the conditional check, so our path-sensitive environment is strengthened by the corresponding guard:

$$
\Gamma_{1} \doteq \Gamma_{0}, \mathrm{i} 1: \operatorname{natN}\langle\mathrm{i} 2+1\rangle, \mathrm{r} 1: \mathrm{B}, \text { i } 2<\operatorname{len}(\mathrm{a})
$$

Finally, the above constraints are solved to:

$$
\kappa_{i 2} \mapsto 0 \leq \nu<\operatorname{len}(a) \quad \kappa_{r 2} \mapsto \text { true }
$$

which verifies that the "callback" $f$ is indeed called with values of type $i d x\langle a\rangle$, as it is only called with i2:idx $\langle a\rangle$, obtained by plugging the solution into the template in (4).

\subsubsection{Mutation}

In the imperative, object-oriented setting (common to dynamic scripting languages), we must account for class and object invariants and their preservation in the presence of field mutation. For example, consider the code in Figure 2, modified from the Octane Navier-Stokes benchmark.

Class Invariants Class Field implements a 2-dimensional vector, "unrolled" into a single array dens, whose size is the product of the width and height fields. We specify this invariant by requiring that width and height be strictly positive (i.e. pos) and that dens be a grid with dimensions specified by this.w and this.h. An advantage of SMT-based refinement typing is that modern SMT solvers support non-linear reasoning, which lets rsc specify and verify program specific invariants outside the scope of generic bounds checkers.

Mutable and Immutable Fields The above invariants are only meaningful and sound if fields $w$ and $h$ cannot be modified after object creation. We specify this via the immutable qualifier, which is used by rsc to then (1) prevent updates to the field outside the constructor, and (2) allow refinements of fields (e.g. dens) to soundly refer to the values of those immutable fields.

Constructors We can create instances of Field, by using new Field(...) which invokes the constructor with the supplied parameters. rsc ensures that at the end of the constructor, the created object actually satisfies all specified class invariants i.e. field refinements. Of course, this only holds if the parameters passed to the constructor satisfy certain preconditions, specified via the input types. Consequently, rsc accepts the first call, but rejects the second: 


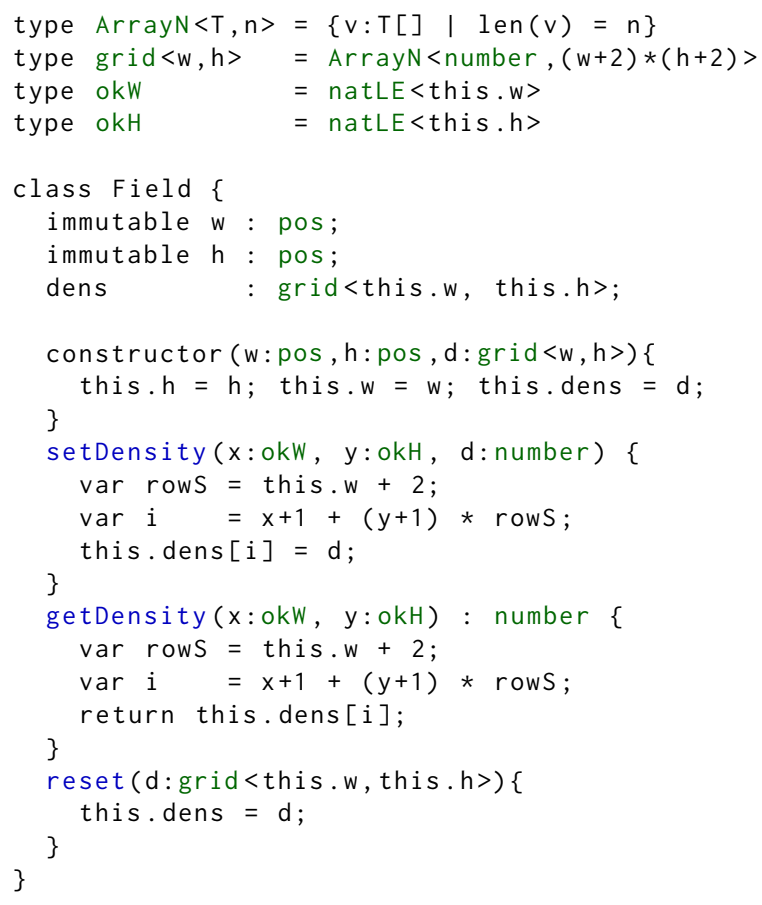

Figure 2: Two-Dimensional Arrays

var $z=$ new Field(3,7, new Array (45)); // OK var $q=$ new Field (3,7, new Array (44)); // BAD

Methods rsc uses class invariants to verify setDensity and getDensity, that are checked assuming that the fields of this enjoy the class invariants, and method inputs satisfy their given types. The resulting VCs are valid and hence, check that the methods are array-safe. Of course, clients must supply appropriate arguments to the methods. Thus, rsc accepts the first call, but rejects the second as the $x$ coordinate 5 exceeds the actual width (i.e. z.w), namely 3 :

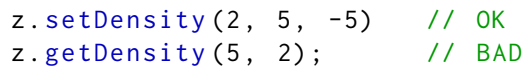

Mutation The dens field is not immutable and hence, may be updated outside of the constructor. However, rsc requires that the class invariants still hold, and this is achieved by ensuring that the new value assigned to the field also satisfies the given refinement. Thus, the reset method requires inputs of a specific size, and updates dens accordingly. Hence:

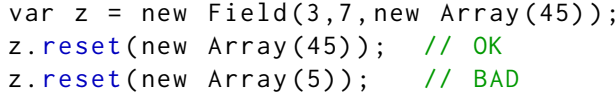

\section{Formal System}

Next, we formalize the ideas outlined in $\S 2$. We introduce our formal core FRSC: an imperative, mutable, objectoriented subset of Refined TypeScript, that closely follows the design of CFJ [25], (the language used to formalize $\mathrm{X} 10)$, which in turn is based on Featherweight Java [18].
To ease refinement reasoning, we translate FRSC to a functional, yet still mutable, intermediate language IRSC. We then formalize our static semantics in terms of IRSC.

\subsection{Formal Language}

\subsubsection{Source Language (FRSC)}

The syntax of this language is given below. Meta-variable e ranges over expressions, which can be variables $\mathrm{x}$, constants c, property accesses e.f, method calls e.m( $\overline{\mathrm{e}})$, object construction new $C(\overline{\mathrm{e}})$, and cast operations $<T>\mathrm{e}$. Statements $\mathrm{s}$ include variable declarations, field updates, assignments, conditionals, concatenations and empty statements. Method declarations include a type signature, specifying input and output types, and a body, i.e. a statement immediately followed by a returned expression. Class definitions distinguish between immutable and mutable members, using $\circ \mathrm{f}: T$ and $\square \mathrm{f}: T$, respectively. As in CFJ, each class and method definition is associated with an invariant $p$.

$$
\begin{aligned}
\mathrm{e} \quad:= & \mathrm{x}|\mathrm{c}| \text { this } \mid \text { e.f } \mid \text { e.m }(\overline{\mathrm{e}}) \mid \text { new } C(\overline{\mathrm{e}}) \mid<T>\mathrm{e} \\
\mathrm{s}::= & \operatorname{var} \mathrm{x}=\mathrm{e}|\mathrm{e} . \mathrm{f}=\mathrm{e}| \mathrm{x}=\mathrm{e} \mid \operatorname{if}(\mathrm{e})\{\mathrm{s}\} \text { else }\{\mathrm{s}\} \mid \\
& \mathrm{s} ; \mathrm{s} \mid \text { skip } \\
\mathrm{B} \quad::= & \mathrm{s} ; \operatorname{return} \mathrm{e} \\
\widetilde{\mathrm{M}}::= & \mathrm{m}(\overline{\mathrm{x}}: \bar{T})\{p\}: T\{\mathrm{~B}\} \\
\mathrm{F} \quad::= & \cdot|\circ f: T| \square f: T \mid \mathrm{F}_{1} ; \mathrm{F}_{2} \\
\widetilde{\mathrm{C}}::= & \operatorname{class} C\{p\} \text { extends } R\{\mathrm{~F}, \widetilde{\mathrm{M}}\}
\end{aligned}
$$

The core system does not formalize: (a) method overloading, which is orthogonal to the current contribution and has been investigated in previous work [38], or (b) method overriding, which means that method names are distinct from the ones defined in parent classes.

\subsubsection{Intermediate Language (IRSC)}

FRSC, while syntactically similar to TS, is not entirely suitable for refinement type checking in its current form, due to features like assignment. To overcome this challenge we translate FRSC to a functional language IRSC through a Static Single Assignment (SSA) transformation, which produces programs that are equivalent (in a sense that we will make precise in the sequel). In IRSC, statements are replaced by let-bindings and new variables are introduced for each variable being reassigned in the respective FRSC code. Thus, IRSC has the following syntax:

$$
\begin{array}{rlrl}
e & := & x|c| \text { this }|e \cdot f| e \cdot m(\bar{e}) \mid \text { new } C(\bar{e}) \mid \\
& e \text { as } T|e \cdot f \leftarrow e| u\langle e\rangle \mid \\
u \quad:= & \langle\rangle \mid \text { let } x=e \text { in }\langle\rangle \mid \\
& \text { letif }\left[\bar{x}, \bar{x}_{1}, \bar{x}_{2}\right](e) ? u_{1}: u_{2} \text { in }\langle\rangle \\
F \quad:= & \cdot|\circ f: T| \square f: T \mid F_{1} ; F_{2} \\
\widetilde{M} \quad:= & \cdot|\operatorname{def} m(\bar{x}: \bar{T})\{p\}: T=e| \widetilde{M}_{1} ; \widetilde{M}_{2} \\
\widetilde{C} \quad:= & \text { class } C\{p\} \triangleleft R\{F ; \widetilde{M}\}
\end{array}
$$

The majority of the expression forms $e$ are unsurprising. An exception is the form of the SSA context $u$, which corresponds to the translation of a statement s and contains a hole \langle\rangle that will hold the translation of the continuation of $\mathrm{s}$. 


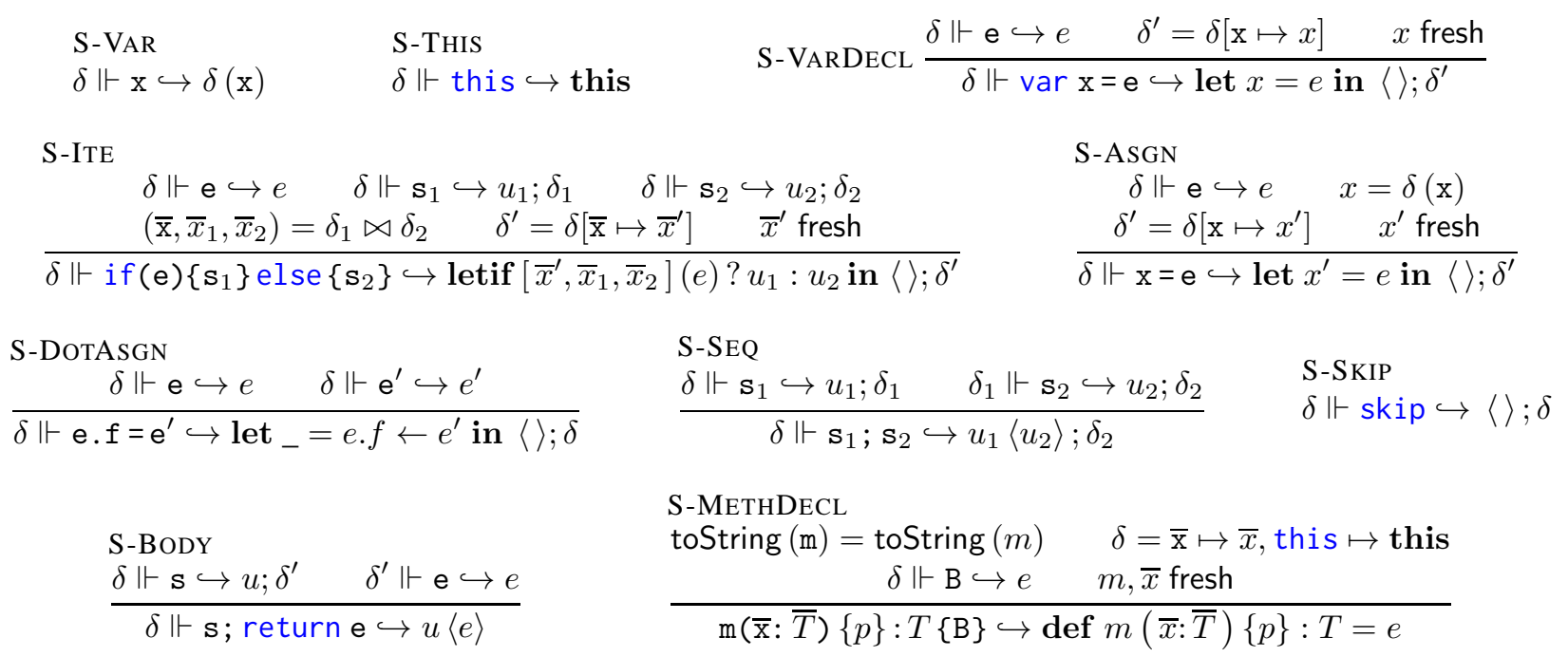

Figure 3: Selected SSA Transformation Rules

SSA Transformation Figure 3 describes the SSA transformation, that uses a translation environment $\delta$, to map FRSC variables $\mathrm{x}$ to IRSC variables $x$. The translation of expressions e to $e$ is routine: as expected, S-VAR maps the source level $x$ to the current binding of $x$ in $\delta$. The translating judgment of statements $\mathrm{s}$ has the form: $\delta \Vdash \mathrm{s} \hookrightarrow u ; \delta^{\prime}$. The output environment $\delta^{\prime}$ is used for the translation of the expression that will fill the hole in $u$.

The most interesting case is that of the conditional statement (rule S-ITE). The conditional expression and each branch are translated separately. To compute variables that get updated in either branch ( $\Phi$-variables), we combine the produced translation states $\delta_{1}$ and $\delta_{2}$ as $\delta_{1} \bowtie \delta_{2}$ defined as:

$$
\left\{\left(\mathrm{x}, x_{1}, x_{2}\right) \mid \mathrm{x} \mapsto x_{1} \in \delta_{1}, \mathrm{x} \mapsto x_{2} \in \delta_{2}, x_{1} \neq x_{2}\right\}
$$

Fresh $\Phi$-variables $\bar{x}^{\prime}$ populate the output SSA environment $\delta^{\prime}$. Along with the versions of the $\Phi$-variables for each branch $\left(\bar{x}_{1}\right.$ and $\left.\bar{x}_{2}\right)$, they are used to annotate the produced structure.

Assignment statements introduce a new SSA variable and bind it to the updated source-level variable (rule SASGN). Statement sequencing is emulated with nesting SSA contexts (rule S-SEQ); empty statements introduce a hole (rule S-SKIP); and, finally, method declarations fill in the hole introduced by the method body with the translation of the return expression (rule S-METHDECL).

\subsubsection{Consistency}

To validate our transformation, we provide a consistency result that guarantees that stepping in the target language preserves the transformation relation, after the program in the source language has made an appropriate number of steps. We define a runtime configuration $\mathrm{R}$ for FRSC (resp. $R$ for IRSC) for a program $\mathrm{P}$ (resp. $P$ ) as:

$$
\begin{array}{ll}
\mathrm{P} \doteq \mathrm{S} ; \mathrm{B} & P \doteq S ; e \\
\mathrm{R} \doteq \mathrm{K} ; \mathrm{B} & R \doteq K ; e \\
\mathrm{~K} \doteq \mathrm{S} ; \mathrm{L} ; \mathrm{X} ; \mathrm{H} & K \doteq S ; H
\end{array}
$$

Runtime state $\mathrm{K}$ consists of the call stack X, the local store of the current stack frame $\mathrm{L}$ and the heap $\mathrm{H}$. The runtime state for IRSC, $R$ only consists of the signatures $S$ and a heap $H$.

We establish the consistency of the SSA transformation by means of a weak forward simulation theorem that connects the dynamic semantics of the two languages. To that end, we define small-step operational semantics for both languages, of the form $\mathrm{R} \longrightarrow \mathrm{R}^{\prime}$ and $R \longrightarrow R^{\prime}$. Figure 12 presents the dynamic behavior of the two languages. Rules for FRSC have been adapted from Rastogi et al. [27]. Note how in rule R-CAST the cast operation reduces to a call to the built-in check function, where $\llbracket T \rrbracket$ encodes type $T$. Rules for IRSC are mostly routine, with the exception of rule R-LETIF: expression $e$ has been produced assuming $\Phi$ variables $\bar{x}$. After the branch has been determined we pick the actual $\Phi$-variables $\left(\bar{x}_{1}\right.$ or $\left.\bar{x}_{2}\right)$ and replace them in $e$. This formulation allows us to perform all the SSA-related bookkeeping in a single reduction step, which is key to preserving our consistency invariant that IRSC steps faster than FRSC.

We also extend our SSA transformation judgment to runtime configurations, leveraging the SSA environments that have been statically computed for each program entity, which now form a global SSA environment $\Delta$, mapping each AST node (e, s, etc.) to an SSA environment $\delta$ :

$$
\Delta::=\cdot|\mathrm{e} \mapsto \delta| \mathrm{s} \mapsto \delta|\ldots| \Delta_{1} ; \Delta_{2}
$$




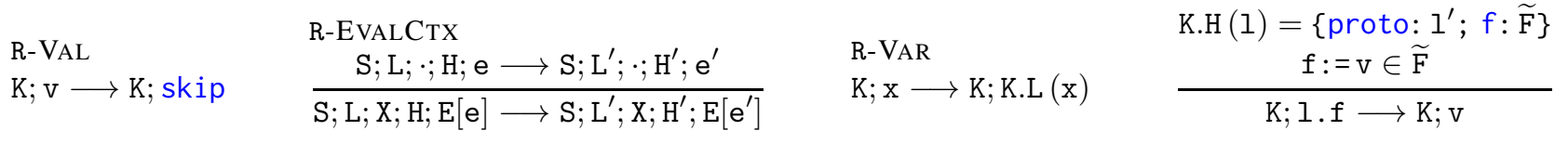

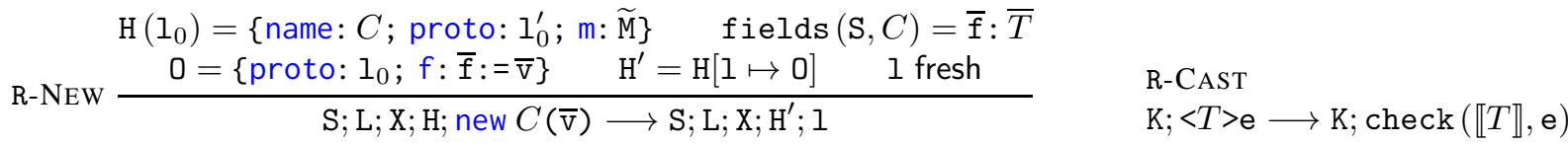

\begin{tabular}{|c|c|c|}
\hline $\begin{array}{l}\text { R-VARDECL } \\
\qquad \mathrm{L}^{\prime}=\mathrm{K} \cdot \mathrm{L}[\mathrm{x} \mapsto \mathrm{v}]\end{array}$ & $\begin{array}{c}\text { resolve_method }(\mathrm{H}, \mathrm{l}, \mathrm{m})=\mathrm{m}(\overline{\mathrm{x}})\{\mathrm{s} ; \text { return e }\} \\
\mathrm{L}^{\prime}=\overline{\mathrm{x}} \mapsto \overline{\mathrm{v}} ; \text { this } \mapsto \mathrm{I} \quad \mathrm{X}^{\prime}=\mathrm{X} ; \mathrm{L}, \mathrm{E}\end{array}$ & $\begin{array}{l}\text { R-DotASGN } \\
\mathrm{H}^{\prime}=\mathrm{K} . \mathrm{H}[\mathrm{l} \mapsto \mathrm{K} . \mathrm{H}(\mathrm{l})[\mathrm{f} \mapsto \mathrm{v}]]\end{array}$ \\
\hline$\overline{\mathrm{K}} ; \operatorname{var} \mathrm{x}=\mathrm{v} \longrightarrow \mathrm{K} \triangleleft \mathrm{L}^{\prime}$; skip & $\longrightarrow \mathrm{S} ; \mathrm{L}^{\prime} ; \mathrm{X}^{\prime} ; \mathrm{H} ; \mathrm{s} ;$ return e & $\mathrm{K} ; \mathrm{l} . \mathrm{f}=\mathrm{v} \longrightarrow \mathrm{K} \triangleleft \mathrm{H}^{\prime} ; \mathrm{v}$ \\
\hline
\end{tabular}

\begin{tabular}{|c|c|c|c|}
\hline & R-ITE & & \\
\hline $\begin{array}{l}\text { R-ASGN } \\
\quad \mathrm{L}^{\prime}=\mathrm{K} \cdot \mathrm{L}[\mathrm{x} \mapsto \mathrm{v}]\end{array}$ & $\begin{array}{c}\mathrm{c}=\text { true } \Rightarrow i=1 \\
\mathrm{c}=\text { false } \Rightarrow i=2\end{array}$ & $\mathrm{~K} . \mathrm{X}=\mathrm{X}^{\prime} ; \mathrm{L}, \mathrm{E}$ & R-SKIP \\
\hline$\overline{\mathrm{K} ; \mathrm{x}=\mathrm{v} \longrightarrow \mathrm{K} \triangleleft \mathrm{L}^{\prime} ; \mathrm{v}}$ & $\overline{\mathrm{K} ; \mathrm{if}(\mathrm{c})\left\{\mathrm{s}_{1}\right\} \text { else }\left\{\mathrm{s}_{2}\right\} \longrightarrow \mathrm{K} ; \mathrm{s}_{i}}$ & $\overline{\mathrm{K} ; \text { return } \mathrm{v} \longrightarrow \mathrm{K} \triangleleft \mathrm{X}^{\prime}, \mathrm{L} ; \mathrm{E}[\mathrm{v}]}$ & \\
\hline
\end{tabular}

\section{Operational Semantics for IRSC}

$$
K ; e \longrightarrow K^{\prime} ; e^{\prime}
$$

\begin{tabular}{|c|c|c|}
\hline $\begin{array}{l}\text { RC-ECTх } \\
\qquad K ; e \longrightarrow K^{\prime} ; e^{\prime}\end{array}$ & $\begin{array}{l}\text { R-FIELD } \\
K . H(l)=\left\{\text { proto: } l^{\prime} ; f: \widetilde{F}\right\} \\
\quad f:=v \in \widetilde{F}\end{array}$ & $\begin{array}{l}\mathrm{R}-\mathrm{CALL} \\
\text { resolveMethod }(H, l, m)=(\operatorname{def} m(\bar{x}: \bar{S})\{p\}: T=e) \\
\qquad \operatorname{eval}([\bar{v} / \bar{x}, l / \text { this }] p)=\text { true }\end{array}$ \\
\hline$\overline{K ; E[e] \longrightarrow K^{\prime} ; E\left[e^{\prime}\right]}$ & $K ; l . f \longrightarrow K ; v$ & $K ; \operatorname{l.m}(\bar{v}) \longrightarrow K ;[\bar{v} / \bar{x}, l /$ this $] e$ \\
\hline
\end{tabular}

R-NEW

$\mathrm{H}\left(l_{0}\right)=\left\{\right.$ name: $C$; proto: $\left.l_{0}^{\prime} ; \mathrm{m}: \widetilde{M}\right\} \quad$ fields $(S, C)=\bar{f}: \bar{T}$

\begin{tabular}{|c|c|c|c|}
\hline $\begin{array}{l}\text { R-DotASGN } \\
H^{\prime}=K . H[l \mapsto K . H(l)[f \mapsto v]]\end{array}$ & $\begin{array}{l}\mathrm{R} \text {-CAST } \\
\Gamma \vdash K(l): S ; S \leq T\end{array}$ & $\begin{array}{l}\text { R-LETIF } \\
\qquad c=\text { true } \Rightarrow i=1\end{array}$ & $c=$ false $\Rightarrow i=2$ \\
\hline$K ; l . f \leftarrow v \longrightarrow K \triangleleft H^{\prime} ; v$ & $\overline{K ; l \text { as } T \longrightarrow K ; l}$ & $\overline{K ; \text { letif }\left[\bar{x}, \bar{x}_{1}, \bar{x}_{2}\right](c) ? u}$ & $\overline{\text { in } e \longrightarrow K ; u_{i}\left\langle\left[\overline{x_{i}} / \bar{x}\right] e\right\rangle}$ \\
\hline
\end{tabular}

$\frac{O=\left\{\text { proto: } l_{0} ; \mathrm{f}: \bar{f}:=\bar{v}\right\} \quad H^{\prime}=H[l \mapsto O] \quad l \text { fresh }}{S ; H ; \text { new } C(\bar{v}) \longrightarrow S ; H^{\prime} ; l} \quad \begin{aligned} & \text { R-LETIN } \\ & K ; \text { let } x=v \text { in } e \longrightarrow K ;[v / x] e\end{aligned}$

Figure 4: Reduction Rules for FRSC (adapted from Safe TypeScript [27]) and IRSC

We assume that the compile-time SSA translation yields this environment as a side-effect (e.g. $\delta \Vdash$ e $\hookrightarrow e$ produces $\mathrm{e} \mapsto \delta$ ) and the top-level program transformation judgment returns the net effect: $\mathrm{P} \hookrightarrow P \rightsquigarrow \Delta$. Hence, the SSA transformation judgment for configurations becomes: $\mathrm{K} ; \mathrm{B} \stackrel{\Delta}{\hookrightarrow} K ; e$. We can now state our consistency theorem as:

Theorem 1 (SSA Consistency). For configurations $\mathrm{R}$ and $R$ and global store typing $\Delta$, if $\mathrm{R} \stackrel{\Delta}{\hookrightarrow} R$, then either both $\mathrm{R}$ and $R$ are terminal, or if for some $R^{\prime}, R \longrightarrow R^{\prime}$, then there exists $\mathrm{R}^{\prime}$ s.t. $\mathrm{R} \longrightarrow{ }^{+} \mathrm{R}^{\prime}$ and $\mathrm{R}^{\prime} \stackrel{\Delta}{\hookrightarrow} R^{\prime}$.

\subsection{Static Semantics}

Having drawn a connection between source and target language we can now describe refinement checking procedure in terms of IRSC.

Types Type annotations on the source language are propagated unaltered through the translation phase. Our type language (shown below) resembles that of existing refinement type systems [19, 25, 28]. A refinement type $T$ may be an existential type or have the form $\{\nu: N \mid p\}$, where $N$ is a class name $C$ or a primitive type $B$, and $p$ is a logical predicate (over some decidable logic) which describes the properties that values of the type must satisfy. Type specifications (e.g. method types) are existential-free, while inferred types may be existentially quantified [20]. 
Figure 5: Static Typing Rules for IRSC

Logical Predicates Predicates $p$ are logical formulas over terms $t$. These terms can be variables $x$, primitive constants $c$, the reserved value variable $\nu$, the reserved variable this to denote the containing object, field accesses $t . f$, uninterpreted function applications $f(\bar{t})$ and applications of terms on built-in operators $b$, such as $==,<,+$, etc.

$$
\begin{aligned}
T, S, R & ::=\exists x: T_{1} \cdot T_{2} \mid\{\nu: N \mid p\} \\
N & ::=C \mid B \\
p & ::=p_{1} \wedge p_{2}|\neg p| t \\
t & ::=x|c| \nu \mid \text { this }|t . f| f(\bar{t}) \mid b(\bar{t})
\end{aligned}
$$

Structural Constraints Following CFJ, we reuse the notion of an Object Constraint System, to encode constraints related to the object-oriented nature of the program. Most of the rules carry over to our system; we defer them to the supplemental material. The key extension in our setting is we partition $C$ has $I$ (that encodes inclusion of an element $I$ in a class $C$ ) into two cases: $C$ hasMut $I$ and $C$ haslmm $I$, to account for elements that may be mutated. These elements can only be fields (i.e. there is no mutation on methods).

Environments And Well-formedness A type environment $\Gamma$ contains type bindings $x: T$ and guard predicates $p$ that encode path sensitivity. $\Gamma$ is well-formed if all of its bindings are well-formed. A refinement type is well-formed in an environment $\Gamma$ if all symbols (simple or qualified) in its logical predicate are (i) bound in $\Gamma$, and (ii) correspond to immutable fields of objects. We omit the rest of the wellformedness rules as they are standard in refinement type systems (details can be found in the supplemental material).
Besides well-formedness, our system's main judgment forms are those for subtyping and refinement typing [19].

Subtyping is defined by the judgment $\Gamma \vdash S \leq T$. The rules are standard among refinement type systems with existential types. For example, the rule for subtyping between two refinement types $\Gamma \vdash\{\nu: N \mid p\} \leq\left\{\nu: N \mid p^{\prime}\right\}$ reduces to a verification condition: $\operatorname{Valid}\left(\llbracket \Gamma \rrbracket \Rightarrow \llbracket p \rrbracket \Rightarrow \llbracket p^{\prime} \rrbracket\right)$, where $\llbracket \Gamma \rrbracket$ is the embedding of environment $\Gamma$ into our logic that accounts for both guard predicates and variable bindings:

$$
\llbracket \Gamma \rrbracket \doteq \bigwedge\{p \mid p \in \Gamma\} \wedge \bigwedge\{[x / \nu] p, \mid x:\{\nu: N \mid p\} \in \Gamma\}
$$

Here, we assume existential types have been simplified to non-existential bindings when they entered the environment. The full set of rules is included in the supplemental material.

Refinement Typing Rules Figure 5 contains most rules of the two forms of our typing judgements: $\Gamma \vdash e: T$ and $\Gamma \vdash u \triangleright \Gamma^{\prime}$. The first form assigns a type $T$ to an expression $e$ under a typing environment $\Gamma$. The second form checks the body of an SSA context $u$ under $\Gamma$ and returns an environment $\Gamma^{\prime}$ of the variables introduced in $u$ that are going to be available in its hole. Below, we discuss the novel rules:

[T-FIELD-I] Immutable object parts can be assigned a more precise type, by leveraging the preservation of their identity. This notion, known as self-strengthening [20, 25], is defined 
with the aid of the strengthening operator $\uparrow$ :

$$
\begin{aligned}
\{\nu: N \mid p\} \oplus p^{\prime} & \doteq\left\{\nu: N \mid p \wedge p^{\prime}\right\} \\
(\exists x: S . T) \oplus p & \doteq \exists x: S .(T \oplus p) \\
\operatorname{self}(T, t) & \doteq T \oplus(\nu=t)
\end{aligned}
$$

[T-FIELD-M] Here we avoid such strengthening, as the value of field $g_{i}$ is mutable, so cannot appear in refinements.

[T-NEw] Similarly, only immutable fields are referenced in the refinement of the inferred type at object construction.

[T-INV] Extracting the method signature using the has operator has already performed the necessary substitutions to account for the specific receiver object.

[T-CAST] Cast operations are checked statically obviating the need for a dynamic check. This rule uses the notion of compatibility subtyping, which is defined as:

Definition 1 (Compatibility Subtype). A type $S$ is a compatibility subtype of a type $T$ under an environment $\Gamma$ (we write $\Gamma \vdash S \lesssim T$ ), iff $\langle S \stackrel{\Gamma}{\rightarrow}\lfloor T\rfloor\rangle=R \neq$ fail with $\Gamma \vdash R \leq T$.

Here, $\lfloor T\rfloor$ extracts the base type of $T$, and $\langle T \stackrel{\Gamma}{\rightarrow} D\rangle$ succeeds when under environment $\Gamma$ we can statically prove $D$ 's invariants. We use the predicate inv $(D, \nu)$ (as in CFJ), to denote the conjunction of the class invariants of $C$ and its supertypes (with the necessary substitutions of this by $\nu)$. We assume that part of these invariants is a predicate that states inclusion in the specific class (instanceof $(\nu, D)$ ). Therefore, we can prove that $T$ can safely be cast to $D$. Formally:

$$
\begin{aligned}
&\langle\{\nu: C \mid p\} \stackrel{\Gamma}{\rightarrow} D\rangle \doteq \begin{cases}D \oplus p & \text { if } \llbracket \Gamma \rrbracket \Rightarrow \llbracket p \rrbracket \Rightarrow \operatorname{inv}(D, \nu) \\
\text { fail } & \text { otherwise }\end{cases} \\
&\langle\exists x: S . T \stackrel{\Gamma}{\rightarrow} D\rangle \doteq \exists x: S \cdot\langle T \stackrel{\Gamma, x: S}{\longrightarrow} D\rangle
\end{aligned}
$$

[T-ASGN] Only mutable fields may be reassigned.

[T-LETIF] To type conditional structures, we first infer a type for the condition and then check each of the branches $u_{1}$ and $u_{2}$, assuming that the condition is true or false, respectively, to achieve path sensitivity. Each branch assigns types to the $\Phi$-variables which compose $\Gamma_{1}$ and $\Gamma_{2}$, and the propagated types for these variables are fresh types operating as upper bounds to their respective bindings in $\Gamma_{1}$ and $\Gamma_{2}$.

\subsection{Type Soundness}

We reuse the operational semantics for IRSC defined earlier, and extend our type checking judgment to runtime locations $l$ with the use of a heap typing $\Sigma$, mapping locations to types:

$$
\frac{\Sigma(l)=T}{\Gamma ; \Sigma \vdash l: T}
$$

We establish type soundness results for IRSC in the form of a subject reduction (preservation) and a progress theorem that connect the static and dynamic semantics of IRSC.
Theorem 2 (Subject Reduction). If (a) $\Gamma ; \Sigma \vdash e: T$, (b) $\Gamma ; K \vdash_{\Sigma} H$, and (c) $H$; $e \longrightarrow H^{\prime} ; e^{\prime}$, then for some $T^{\prime}$ and $\Sigma^{\prime} \supseteq \Sigma$ : (i) $\Gamma$; $\Sigma^{\prime} \vdash e^{\prime}: T^{\prime}$, (ii) $\Gamma \vdash T^{\prime} \lesssim T$, and (iii) $\Gamma ; K \vdash_{\Sigma^{\prime}} H^{\prime}$.

Theorem 3 (Progress). If $\Gamma ; \Sigma \vdash e: T$ and $\Gamma ; K \vdash_{\Sigma} H$, then either $e$ is a value, or there exist $e^{\prime}, H^{\prime}$ and $\Sigma^{\prime} \supseteq \Sigma$ s.t. $\Gamma ; K \vdash_{\Sigma^{\prime}} H^{\prime}$ and $H ; e \longrightarrow H^{\prime} ; e^{\prime}$.

We defer the proofs to the supplementary material. As a corollary of the progress theorem we get that cast operators are guaranteed to succeed, hence they can safely be removed.

Corollary 4 (Safe Casts). Cast operations can safely be erased when compiling to executable code.

With the use of our Consistency Theorem (Theorem 1) and extending our checking judgment for terms in IRSC to runtime configurations $(\vdash R)$, we can state a soundness result for FRSC:

Theorem 5. (FRSC Type Safety) If $\mathrm{R} \stackrel{\Delta}{\hookrightarrow} R$ and $\vdash R$ then either $\mathrm{R}$ is a terminal form, or there exists $\mathrm{R}$ s.t. $\mathrm{R} \longrightarrow \mathrm{R}$.

\section{Scaling to TypeScript}

TypeScript (TS) extends JavaScript (JS) with modules, classes and a lightweight type system that enables IDE support for auto-completion and refactoring. TS deliberately eschews soundness [3] for backwards compatibility with existing JS code. In this section, we show how to use refinement types to regain safety, by presenting the highlights of Refined TypeScript (and our tool rsc), that scales the core calculus from $\S 3$ up to TS by extending the support for types (\$4.1), reflection (\$4.2), interface hierarchies (\$4.3), and imperative programming $(\$ 4.4)$.

\subsection{Types}

First, we discuss how rsc handles core TS features like object literals, interfaces and primitive types.

Object literal types TS supports object literals, i.e. anonymous objects with field and method bindings. rsc types object members in the same way as class members: method signatures need to be explicitly provided, while field types and mutability modifiers are inferred based on use, e.g. in:

var point $=\{x: 1, y: 2\} ;$ point.x $=2$;

the field $x$ is updated and hence, $r s c$ infers that $x$ is mutable.

Interfaces TS supports named object types in the form of interfaces, and treats them in the same way as their structurally equivalent class types. For example, the interface:

interface Point $\{$ number $x, y ;\}$

is equivalent to a class PointC defined as:

class PointC $\{$ number $x, y ;\}$

In rsc these two types are not equivalent, as objects of type Point I do not necessarily have PointC as their constructor: 
var $\mathrm{pI}=\{\mathrm{x}: 1, \mathrm{y}: 2\}, \mathrm{pC}=$ new PointC $(1,2)$; pI instanceof PointC; // returns false pC instanceof PointC; // returns true

However, $\vdash$ PointC $\leq$ PointI i.e. instances of the class may be used to implement the interface.

Primitive types We extend rsc's support for primitive types to model the corresponding types in TS. TS has undefined and null types to represent the eponymous values, and treats these types as the "bottom" of the type hierarchy, effectively allowing those values to inhabit every type via subtyping. rsc also includes these two types, but does not treat them "bottom" types. Instead rsc handles them as distinct primitive types inhabited solely by undefined and null, respectively, that can take part in unions. Consequently, the following code is accepted by TS but rejected by rsc:

$\operatorname{var} \mathrm{x}=$ undefined; $\operatorname{var} \mathrm{y}=\mathrm{x}+1$;

Unsound Features TS has several unsound features deliberately chosen for backwards compatibility. These include (1) treating undefined and null as inhabitants of all types, (2) co-variant input subtyping, (3) allowing unchecked overloads, and (4) allowing a special "dynamic" any type to be ascribed to any term. rsc ensures soundness by (1) performing checks when non-null (non-undefined) types are required (e.g. during field accesses), (2) using the correct variance for functions and constructors, (3) checking overloads via twophase typing (\$2.1.2), and, (4) eliminating the any type.

Many uses of any (indeed, all uses, in our benchmarks §5) can be replaced with a combination of union or intersection types or downcasting, all of which are soundly checked via path-sensitive refinements. In future work, we wish to support the full language, namely allow dynamically checked uses of any by incorporating orthogonal dynamic techniques from the contracts literature. We envisage a dynamic cast operation cast $_{T}::(x$ : any $) \Rightarrow\{\nu: T \mid \nu=x\}$. It is straightforward to implement cast $_{T}$ for first-order types $T$ as a dynamic check that traverses the value, testing that its components satisfy the refinements [30]. Wrapper-based techniques from the contracts/gradual typing literature should then let us support higher-order types.

\subsection{Reflection}

JS programs make extensive use of reflection via "dynamic" type tests. rsc statically accounts for these by encoding type-tags in refinements. The following tests if $x$ is a number before performing an arithmetic operation on it:

var $r=1$; if (typeof $x===$ "number") $r+=x$;

We account for this idiomatic use of typeof by statically tracking the "type" tag of values inside refinements using uninterpreted functions (akin to the size of an array). Thus, values $v$ of type boolean, number, string, etc. are refined with the predicate $\operatorname{ttag}(v)=$ "boolean", $\operatorname{ttag}(v)=$ "number", ttag $(v)=$ "string", etc., respectively. Furthermore, typeof has type $(z: A) \Rightarrow\{v$ :string $\mid v=\operatorname{ttag}($ $z)$ ) so the output type of typeof $x$ and the path-sensitive guard under which the assignment $r=x+1$ occurs, ensures that at the assignment $x$ can be statically proven to be a number. The above technique coupled with two-phase typing (\$2.1.2) allows rsc to statically verify reflective, valueoverloaded functions that are ubiquitous in TS.

\subsection{Interface Hierarchies}

JS programs frequently build up object hierarchies that represent unions of different kinds of values, and then use value tests to determine which kind of value is being operated on. In TS this is encoded by building up a hierarchy of interfaces, and then performing downcasts based on value tests ${ }^{1}$.

Implementing Hierarchies with bit-vectors The following describes a slice of the hierarchy of types used by the TypeScript compiler (tsc) v1.0.1.0:

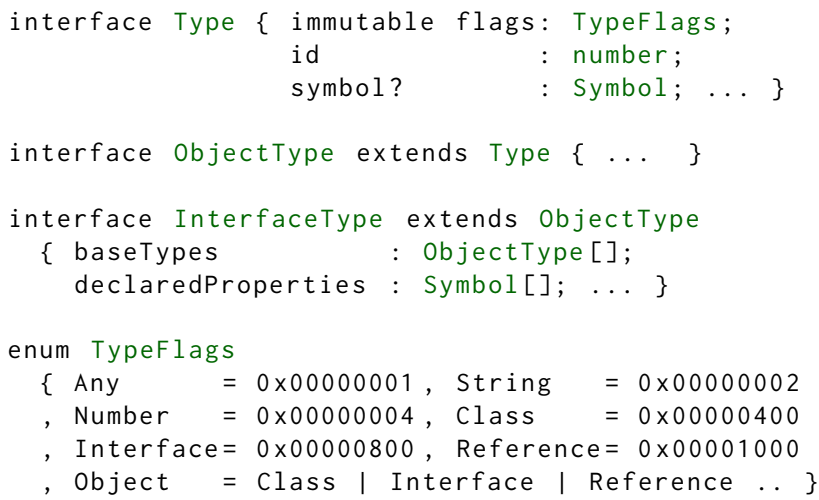

tsc uses bit-vector valued flags to encode membership within a particular interface type, i.e. discriminate between the different entities. (Older versions of tsc used a class-based approach, where inclusion could be tested via instanceof tests.) For example, the enumeration TypeFlags above maps semantic entities to bit-vector values used as masks that determine inclusion in a sub-interface of Type. Suppose $t$ of type Type. The invariant here is that if $t$. flags masked with $0 \times 00000800$ is non-zero, then $t$ can be safely treated as an InterfaceType value, or an ObjectType value, since the relevant flag emerges from the bit-wise disjunction of the Interface flag with some other flags.

Specifying Hierarchies with Refinements rsc allows developers to create and use Type objects with the above invariant by specifying a predicate typeInv ${ }^{2}$ :

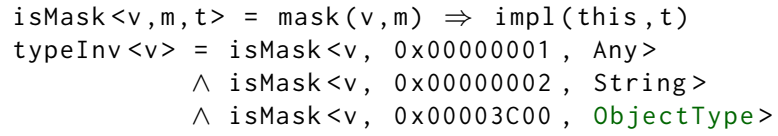

and then refining TypeFlags with the predicate

type TypeFlags $=\{v:$ TypeFlags $\mid$ typeInv $<v>\}$

\footnotetext{
${ }^{1}$ rsc handles other type tests, e.g. instanceof, via an extension of the technique used for typeof tests; we omit a discussion for space.

${ }^{2}$ Modern SMT solvers easily handle formulas over bit-vectors, including operations that shift, mask bit-vectors, and compare them for equality.
} 
Intuitively, the refined type says that when $v$ (that is the flags field) is a bit-vector with the first position set to 1 the corresponding object satisfies the Any interface, etc.

Verifying Downcasts rsc verifies the code that uses ad-hoc hierarchies such as the above by proving the TS downcast operations (that allow objects to be used at particular instances) safe. For example, consider the following code that tests if $\mathrm{t}$ implements the ObjectType interface before performing a downcast from type Type to ObjectType that permits the access of the latter's fields:

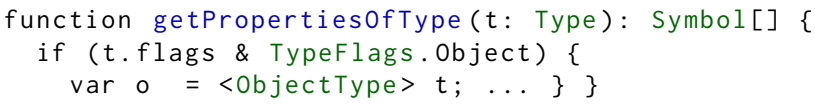

tsc erases casts, thereby missing possible runtime errors. The same code without the if-test, or with a wrong test would pass the TypeScript type checker. rsc, on the other hand, checks casts statically. In particular, <0bjectType $>t$ is treated as a call to a function with signature:

$$
(x:\{A \mid \text { impl }(x, \text { ObjectType })\}) \Rightarrow\{v: \text { ObjectType } \mid v=x\}
$$

The if-test ensures that the immutable field $t$. flags masked with $0 \times 00003 \mathrm{C00}$ is non-zero, satisfying the third line in the type definition of typeInv, which, in turn implies that $t$ in fact implements the ObjectType interface.

\subsection{Imperative Features}

Immutability Guarantees Our system uses ideas from Immutability Generic Java [41] (IGJ) to provide statically checked immutability guarantees. In IGJ a type reference is of the form $\mathrm{C}<\mathrm{M}, \overline{\mathrm{T}}>$, where immutability argument $\mathrm{M}$ works as proxy for the immutability modifiers of the contained fields (unless overridden). It can be one of: Immutable (or IM), when neither this reference nor any other reference can mutate the referenced object; Mutable (or MU), when this and potentially other references can mutate the object; and ReadOnly (or RO), when this reference cannot mutate the object, but some other reference may. Similar reasoning holds for method annotations. IGJ provides deep immutability, since a class's immutability parameter is (by default) reused for its fields; however, this is not a firm restriction imposed by refinement type checking.

Arrays TS's definitions file provides a detailed specification for the Array interface. We extend this definition to account for the mutating nature of certain array operations:

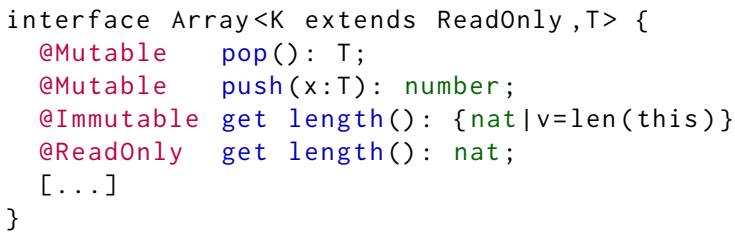

Mutating operations (push, pop, field updates) are only allowed on mutable arrays, and the type of a. length encodes the exact length of an immutable array a, and just a natural number otherwise. For example, assume the following code:

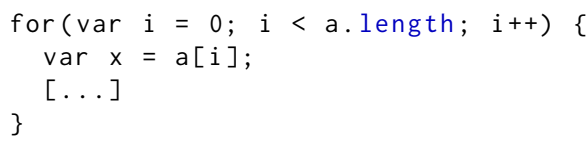

To prove the access $a[i]$ safe we need to establish $0 \leq i$ and $i<a$.length. To guarantee that the length of $a$ is constant, a needs to be immutable, so TypeScript will flag an error unless a: Array<IM, T>.

Object initialization Our formal core $(\$ 3)$ treats constructor bodies in a very limiting way: object construction is merely an assignment of the constructor arguments to the fields of the newly created object. In rsc we relax this restriction in two ways: (a) We allow class and field invariants to be violated within the body of the constructor, but checked for at the exit. (b) We permit the common idiom of certain fields being initialized outside the constructor, via an additional mutability variant that encodes reference uniqueness. In both cases, we still restrict constructor code so that it does not leak references of the constructed object (this) or read any of its fields, as they might still be in an uninitialized state.

(a) Internal Initialization: Constructors Type invariants do not hold while the object is being "cooked" within the constructor. To safely account for this idiom, rsc defers the checking of class invariants (i.e. the types of fields) by replacing: (a) occurrences of this. $f_{i} \leftarrow \mathrm{e}_{i}$, with ${ }_{-} f_{i}=\mathrm{e}_{i}$, where $f_{i}$ are local variables, and (b) all return points with a call ctor_init $\left(\overline{f_{i}}\right)$, where the signature for ctor_init is: $(\bar{f}: \bar{T}) \Rightarrow$ void. Thus, rsc treats field initialization in a field- and path-sensitive way (through the usual SSA conversion), and establishes the class invariants via a single atomic step at the constructor's exit (return).

(b) External Initialization: Unique References Sometimes we want to allow immutable fields to be initialized outside the constructor. Consider the code (adapted from tsc):

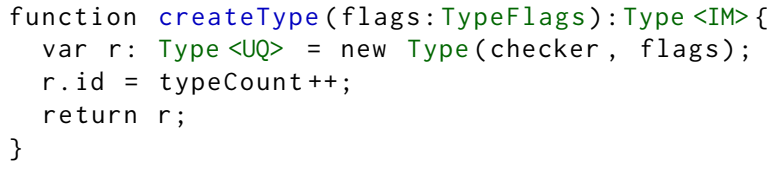

Field id is expected to be immutable. However, its initialization happens after Type's constructor has returned. Fixing the type of $r$ to Type $<I M>$ right after construction would disallow the assignment of the id field on the following line. So, instead, we introduce Unique (or UQ), a new mutability type that denotes that the current reference is the only reference to a specific object, and hence, allows mutations to its fields. When createType returns, we can finally fix the mutability parameter of $r$ to IM. We could also return Type<UQ>, extending the cooking phase of the current object and allowing further initialization by the caller. UQ references obey stricter rules to avoid leaking of unique references:

- they cannot be re-assigned,

- they cannot be generally referenced, unless this occurs at a context that guarantees that no aliases will be produced, 
e.g. the context of e 1 in e1.f $=\mathrm{e} 2$, or the context of a returned expression, and

- they cannot be cast to types of a different mutability (e.g. $<$ C $<$ IM $>>x$ ), as this would allow the same reference to be subsequently aliased.

More expressive initialization approaches are discussed in $\S 6$.

\section{Evaluation}

To evaluate rsc, we have used it to analyze a suite of JS and TS programs, to answer two questions: (1) What kinds of properties can be statically verified for real-world code? (2) What kinds of annotations or overhead does verification impose? Next, we describe the properties, benchmarks and discuss the results.

Safety Properties We verify with rsc the following:

- Property Accesses rsc verifies each field (x.f) or method lookup (x.m (. . )) succeeds. Recall that undefined and null are not considered to inhabit the types to which the field or methods belong,

- Array Bounds rsc verifies that each array read (x[i]) or write $(x[i]=e)$ occurs within the bounds of $x$,

- Overloads rsc verifies that functions with overloaded (i.e. intersection) types correctly implement the intersections in a path-sensitive manner as described in (\$2.1.2).

- Downcasts rsc verifies that at each TS (down)cast of the form $\langle T\rangle$ e, the expression $e$ is indeed an instance of $\mathrm{T}$. This requires tracking program-specific invariants, e.g. bit-vector invariants that encode hierarchies (\$4.3).

\subsection{Benchmarks}

We took a number of existing JS or TS programs and ported them to rsc. We selected benchmarks that make heavy use of language constructs connected to the safety properties described above. These include parts of the Octane test suite, developed by Google as a JavaScript performance benchmark [12] and already ported to TS by Rastogi et al. [27], the TS compiler [22], and the D3 [4] and Transducers libraries [7]:

- navier-stokes which simulates two-dimensional fluid motion over time; richards, which simulates a process scheduler with several types of processes passing information packets; splay, which implements the splay tree data structure; and raytrace, which implements a raytracer that renders scenes involving multiple lights and objects; all from the Octane suite,

- transducers a library that implements composable data transformations, a JavaScript port of Hickey's Clojure library, which is extremely dynamic in that some functions have 12 (value-based) overloads,

\begin{tabular}{l|r|rrr|r} 
Benchmark & LOC & T & M & R & Time (s) \\
\hline navier-stokes & 366 & 3 & 18 & 39 & 473 \\
splay & 206 & 18 & 2 & 0 & 6 \\
richards & 304 & 61 & 5 & 17 & 7 \\
raytrace & 576 & 68 & 14 & 2 & 15 \\
transducers & 588 & 138 & 13 & 11 & 12 \\
d3-arrays & 189 & 36 & 4 & 10 & 37 \\
tsc-checker & 293 & 10 & 48 & 12 & 62 \\
\hline TOTAL & 2522 & 334 & 104 & 91 &
\end{tabular}

Figure 6: LOC is the number of non-comment lines of source (computed via cloc v1.62). The number of RSC specifications given as JML style comments is partitioned into $\mathbf{T}$ trivial annotations i.e. TypeScript type signatures, $\mathbf{M}$ mutability annotations, and $\mathbf{R}$ refinement annotations, i.e. those which actually mention invariants. Time is the number of seconds taken to analyze each file.

- d3-arrays the array manipulating routines from the D3 [4] library, which makes heavy use of higher order functions as well as value-based overloading,

- tsc-checker which includes parts of the TS compiler (v1.0.1.0), abbreviated as tsc. We check 15 functions from compiler/core.ts and 14 functions from compiler/checker.ts (for which we needed to import 779 lines of type definitions from compiler/types.ts). These code segments were selected among tens of thousands of lines of code comprising the compiler codebase, as they exemplified interesting properties, like the bitvector based type hierarchies explained in $\$ 4.3$.

Results Figure 6 quantitatively summarizes the results of our evaluation. Overall, we had to add about 1 line of annotation per 5 lines of code (529 for 2522 LOC). The vast majority $(334 / 529$ or $63 \%)$ of the annotations are trivial, i.e. are TS-like types of the form ( $x:$ nat) $\Rightarrow$ nat; $20 \%(104 / 529)$ are trivial but have mutability information, and only $17 \%$ (91/529) mention refinements, i.e. are definitions like type nat $=\{v:$ number $\mid 0 \leq \mathrm{v}\}$ or dependent signatures like $(\mathrm{a}: \mathrm{T}$ []$, n: i d x<a>) \Rightarrow T$. These numbers show rsc has annotation overhead comparable with TS, as in $83 \%$ cases the annotations are either identical to TS annotations or to TS annotations with some mutability modifiers. Of course, in the remaining $17 \%$ cases, the signatures are more complex than the (non-refined) TS version.

Code Changes We had to modify the source in various small (but important) ways in order to facilitate verification. The total number of changes is summarized in Figure 7. The trivial changes include the addition of type annotations (accounted for above), and simple transforms to work around current limitations of our front end, e.g. converting $\mathrm{x}++$ to $\mathrm{x}=\mathrm{x}+1$. The important classes of changes are the following:

- Control-Flow: Some programs had to be restructured to work around rsc's currently limited support for certain 


\begin{tabular}{l|r|r|r} 
Benchmark & LOC & ImpDiff & AllDiff \\
\hline navier-stokes & 366 & 79 & 160 \\
splay & 206 & 58 & 64 \\
richards & 304 & 52 & 108 \\
raytrace & 576 & 93 & 145 \\
transducers & 588 & 170 & 418 \\
d3-arrays & 189 & 8 & 110 \\
tsc-checker & 293 & 9 & 47 \\
\hline TOTAL & 2522 & 469 & 1052
\end{tabular}

Figure 7: LOC is the number of non-comment lines of source (computed via cloc v1.62). The number of lines at which code was changed, which is counted as either: ImpDiff: the important changes that require restructuring the original JavaScript code to account for limited support for control flow constructs, to replace records with classes and constructors, and to add ghost functions, or, AllDiff: the above plus trivial changes due to the addition of plain or refined type annotations (Figure 6), and simple edits to work around current limitations of our front end.

control flow structures (e.g. break). We also modified some loops to use explicit termination conditions.

- Classes and Constructors: As rsc does not yet support default constructor arguments, we modified relevant new calls in Octane to supply those explicitly. We also refactored navier-stokes to use traditional OO style classes and constructors instead of JS records with functionvalued fields.

- Non-null Checks: In splay we added 5 explicit nonnull checks for mutable objects as proving those required precise heap analysis that is outside rsc's scope.

- Ghost Functions: navier-stokes has more than a hundred (static) array access sites, most of which compute indices via non-linear arithmetic (i.e. via computed indices of the form $\operatorname{arr}[r * s+c])$; SMT support for non-linear integer arithmetic is brittle (and accounts for the anomalous time for navier-stokes). We factored axioms about non-linear arithmetic into ghost functions whose types were proven once via non-linear SMT queries, and which were then explicitly called at use sites to instantiate the axioms (thereby bypassing non-linear analysis). An example of such a function is:

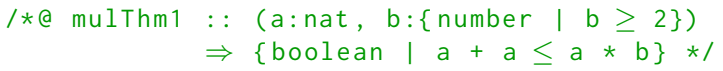

which, when instantiated via a call mulThm $(x, y)$ establishes the fact that (at the call-site), $x+x \leq x * y$. The reported performance assumes the use of ghost functions. In the cases where they were not used RSC would time out.

\subsection{Transducers (A Case Study)}

We now delve deeper into one of our benchmarks: the Transducers library. At its heart this library is about reducing col- lections, aka performing folds. A Transformer is anything that implements three functions: init to begin computation, step to consume one element from an input collection, and result to perform any post-processing. One could imagine rewriting reduce from Figure 1 by building a Transformer where init returns $x$, step invokes $f$, and result is the identity. ${ }^{3}$ The Transformers provided by the library are composable - their constructors take, as a final argument, another Transformer, and then all calls to the outer Transformer's functions invoke the corresponding one of the inner Transformer. This gives rise to the concept of a Transducer, a function of type Transformer $\Rightarrow$ Transformer and this library's namesake.

The main reason this library interests us is because some of its functions are massively overloaded. Consider, for example, the reduce function it defines. As discussed above, reduce needs a Transformer and a collection. There are two opportunities for overloading here. First of all, the main ways that a Transformer is more general than a simple step function is that it can be stateful and that it defines the result post-processing step. Most of the time the user does not need these features, in which case their Transformer is just a wrapper around a step function. Thus for convenience, the user is allowed to pass in either a full-fledged Transformer or a step function which will automatically get wrapped into one. Secondly, the collection being reduced can be a stunning array of options: an Array, a string (i.e. a collection of characters, which are themselves just strings), an arbitrary object (i.e., in JS, a collection of key-value pairs), an iterator (an object that defines a next function that iterates through the collection), or an iterable (an object that defines an iterator function that returns an iterator). Each of these collections needs to be dispatched to a type-specific reduce function that knows how to iterate over that kind of collection. In each overload, the type of the collection must match the type of the Transformer or step function. Thus our reduce begins as shown in Figure 8:

If you count all 5 types of collection and the 2 options for step function vs Transformer, this function has 10 distinct overloads! Another similar function offers 5 choices of input collection and 3 choices of output collection for a total of 15 distinct overloads.

\subsection{Unhandled Cases}

This section outlines some cases that RSC fails to handle and explains the reasons behind them.

Complex Constructor Patterns Due to our limited internal initialization scheme, there are certain common constructor patterns that are not supported by RSC. For example, the code below:

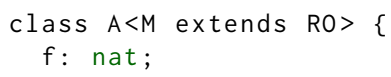

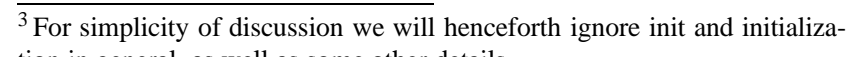
tion in general, as well as some other details. 


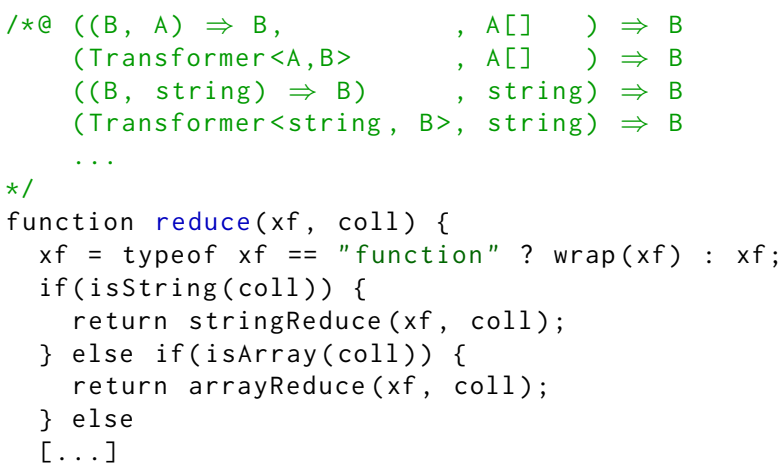

Figure 8: Adapted sample from Transducers benchmark

constructor () $\{$ this.setF (1); \} \}

Currently, RSC does not allow method invocations on the object under construction in the constructor, as it cannot track the (value of the) updates happening in the method setF. Note that this case is supported by IGJ. The relevant section in the related work (\$6) includes approaches that could lift this restriction.

Recovering Unique References RSC cannot recover the Unique state for objects after they have been converted to Mutable (or other state), as it lacks a fine-grained alias tracking mechanism. Assume, for example the function distict below from the TS compiler v1.0.1.0:

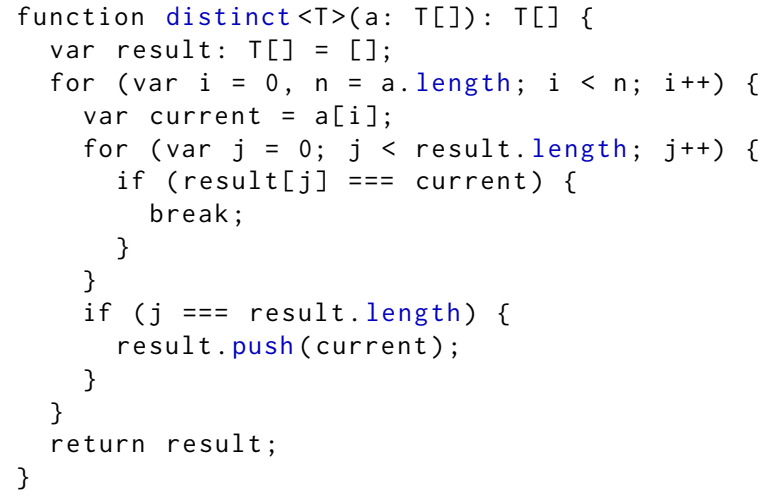

The results array is defined at line 2 so it is initially typed as Array<UQ, T>. At lines 5-9 it is iterated over, so in order to prove the access at line 6 safe, we need to treat results as an immutable array. However, later on at line 11 the code pushes an element onto results, an operation that requires a mutable receiver. Our system cannot handle the interleaving of these two kinds of operations that (in addition) appear in a tight loop (lines 3-13). The alias tracking section in the related work ( $\$ 6)$ includes approaches that could allow support for such cases.

Annotations per Function Overload A weakness of RSC, that stems from the use of Two-Phased Typing [38] in han- dling intersection types, is cases where type checking requires annotations under a specific signature overload. Consider for example the following code, which is a variation of the reduce function presented in $\S 2$ :

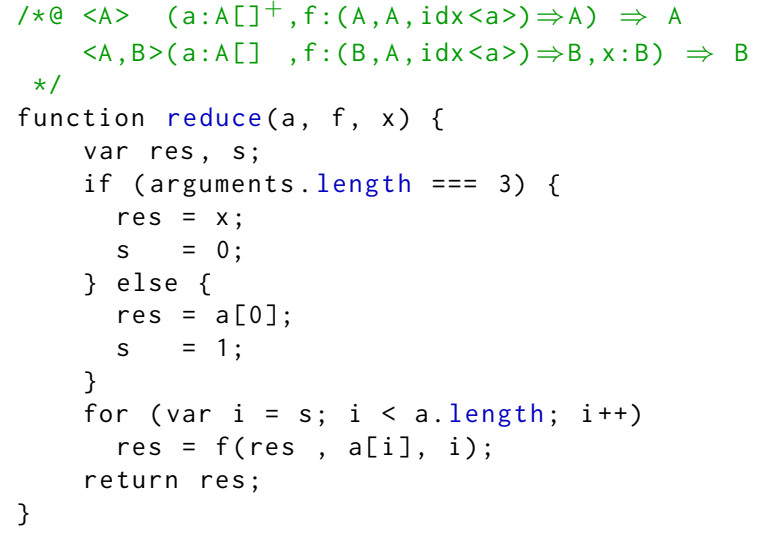

Checking the function body for the second overload (line 2) is problematic: without a user type annotation on res, the inferred type after joining the environments of each conditional branch will be res: $B+(A+$ undefined) (as res is collecting values from $x$ and $a[0]$, at lines 7 and 10 , respectively), instead of the intended res: $B$. This causes an error when res is passed to function $f$ at line 14, expected to have type $B$, which cannot be overcome even with refinement checking, since this code is no longer executed under the check on the length of the arguemnts variable (line 6). A solution to this issue would be for the user to annotate the type of res as B at its definition at line 5, but only for the specific (second) overload. The assignment at line 10 will be invalid, but this is acceptable since that branch is provably (by the refinement checking phase [38]) dead. This option, however, is currently not available.

\section{Related Work}

$\mathrm{RSC}$ is related to several distinct lines of work.

Types for Dynamic Languages Original approaches incorporate flow analysis in the type system, using mechanisms to track aliasing and flow-sensitive updates [1, 35]. Typed Racket's occurrence typing narrows the type of unions based on control dominating type tests, and its latent predicates lift the results of tests across higher order functions [36]. DRuby [10] uses intersection types to represent summaries for overloaded functions. TeJaS [21] combines occurrence typing with flow analysis to analyze JS [21]. Unlike RSC none of the above reason about relationships between values of multiple program variables, which is needed to account for value-overloading and richer program safety properties.

Program Logics At the other extreme, one can encode types as formulas in a logic, and use SMT solvers for all the analysis (subtyping). DMinor explores this idea in a first-order functional language with type tests [2]. The idea can be scaled to higher-order languages by embedding the typing 
relation inside the logic [6]. DJS combines nested refinements with alias types [31], a restricted separation logic, to account for aliasing and flow-sensitive heap updates to obtain a static type system for a large portion of JS [5]. DJS proved to be extremely difficult to use. First, the programmer had to spend a lot of effort on manual heap related annotations; a task that became especially cumbersome in the presence of higher order functions. Second, nested refinements precluded the possibility of refinement inference, further increasing the burden on the user. In contrast, mutability modifiers have proven to be lightweight [41] and two-phase typing lets rsc use liquid refinement inference [28], yielding a system that is more practical for real world programs. Extended Static Checking [9] uses Floyd-Hoare style firstorder contracts (pre-, post-conditions and loop invariants) to generate verification conditions discharged by an SMT solver. Refinement types can be viewed as a generalization of Floyd-Hoare logics that uses types to compositionally account for polymorphic higher-order functions and containers that are ubiquitous in modern languages like TS.

$\mathrm{X} 10$ [25] is a language that extends an object-oriented type system with constraints on the immutable state of classes. Compared to X10, in RSC: (a) we make mutability parametric [41], and extend the refinement system accordingly, (b) we crucially obtain flow-sensitivity via SSA transformation, and path-sensitivity by incorporating branch conditions, (c) we account for reflection by encoding tags in refinements and two-phase typing [38], and (d) our design ensures that we can use liquid type inference [28] to automatically synthesize refinements.

Analyzing TypeScript Feldthaus et al. present a hybrid analysis to find discrepancies between TS interfaces [40] and their JS implementations [8], and Rastogi et al. extend TS with an efficient gradual type system that mitigates the unsoundness of TS's type system [27].

Object and Reference Immutability rsc builds on existing methods for statically enforcing immutability. In particular, we build on Immutability Generic Java (IGJ) which encodes object and reference immutability using Java generics [41]. Subsequent work extends these ideas to allow (1) richer ownership patterns for creating immutable cyclic structures [42], (2) unique references, and ways to recover immutability after violating uniqueness, without requiring an alias analysis [13].

Reference immutability has recently been combined with rely-guarantee logics (originally used to reason about thread interference), to allow refinement type reasoning. Gordon $e t$ al. [14] treat references to shared objects like threads in relyguarantee logics, and so multiple aliases to an object are allowed only if the guarantee condition of each alias implies the rely condition for all other aliases. Their approach allows refinement types over mutable data, but resolving their proof obligations depends on theorem-proving, which hinders automation. Militão et al. [23] present Rely-Guarantee
Protocols that can model complex aliasing interactions, and, compared to Gordon's work, allow temporary inconsistencies, can recover from shared state via ownership tracking, and resort to more lightweight proving mechanisms.

The above extensions are orthogonal to rsc; in the future, it would be interesting to see if they offer practical ways for accounting for (im)mutability in TS programs.

Object Initialization A key challenge in ensuring immutability is accounting for the construction phase where fields are initialized. We limit our attention to lightweight approaches i.e. those that do not require tracking aliases, capabilities or separation logic [11, 31]. Haack and Poll [17] describe a flexible initialization schema that uses secret tokens, known only to stack-local regions, to initialize all members of cyclic structures. Once initialization is complete the tokens are converted to global ones. Their analysis is able to infer the points where new tokens need to be introduced and committed. The Masked Types approach tracks, within the type system, the set of fields that remain to be initialized [26]. X10's hardhat flow-analysis based approach to initialization [43] and Freedom Before Commitment [32] are perhaps the most permissive of the lightweight methods, allowing, unlike rsc, method dispatches or field accesses in constructors.

\section{Conclusions and Future Work}

We have presented RSC which brings SMT-based modular and extensible analysis to dynamic, imperative, classbased languages by harmoniously integrating several techniques. First, we restrict refinements to immutable variables and fields (cf. X10 [34]). Second, we make mutability parametric (cf. IGJ [41]) and recover path- and flow-sensitivity via SSA. Third, we account for reflection and value overloading via two-phase typing [38]. Finally, our design ensures that we can use liquid type inference [28] to automatically synthesize refinements. Consequently, we have shown how rsc can verify a variety of properties with a modest annotation overhead similar to TS. Finally, our experience points to several avenues for future work, including: (1) more permissive but lightweight techniques for object initialization [43], (2) automatic inference of trivial types via flow analysis [16], (3) verification of security properties, e.g. access-control policies in JS browser extensions [15]. 


\section{References}

[1] C. Anderson, P. Giannini, and S. Drossopoulou. Towards Type Inference for Javascript. In Proceedings of the 19th European Conference on Object-Oriented Programming, 2005.

[2] G. M. Bierman, A. D. Gordon, C. Hriţcu, and D. Langworthy. Semantic Subtyping with an SMT Solver. In Proceedings of the 15th ACM SIGPLAN International Conference on Functional Programming, 2010.

[3] G. M. Bierman, M. Abadi, and M. Torgersen. Understanding typescript. In ECOOP 2014 - Object-Oriented Programming - 28th European Conference, Uppsala, Sweden, July 28 August 1, 2014. Proceedings, pages 257-281, 2014.

[4] M. Bostock. http://d3js.org/.

[5] R. Chugh, D. Herman, and R. Jhala. Dependent types for javascript. In Proceedings of the ACM International Conference on Object Oriented Programming Systems Languages and Applications, OOPSLA '12, pages 587-606, New York, NY, USA, 2012. ACM.

[6] R. Chugh, P. M. Rondon, and R. Jhala. Nested Refinements: A Logic for Duck Typing. In Proceedings of the 39th Annual ACM SIGPLAN-SIGACT Symposium on Principles of Programming Languages, 2012.

[7] Cognitect Labs. https://github.com/cognitect-labs/transducers-bjesg, Berlin, Heidelberg, 2014.

[8] A. Feldthaus and A. Møller. Checking Correctness of TypeScript Interfaces for JavaScript Libraries. In Proceedings of the ACM International Conference on Object Oriented Programming Systems Language and Applications, 2014.

[9] C. Flanagan, K. R. M. Leino, M. Lillibridge, G. Nelson, J. B. Saxe, and R. Stata. Extended static checking for java. In Proceedings of the ACM SIGPLAN 2002 Conference on Programming Language Design and Implementation, PLDI '02, pages 234-245, New York, NY, USA, 2002. ACM. ISBN 158113-463-0.

[10] M. Furr, J.-h. D. An, J. S. Foster, and M. Hicks. Static Type Inference for Ruby. In Proceedings of the 2009 ACM Symposium on Applied Computing, 2009.

[11] P. Gardner, S. Maffeis, and G. D. Smith. Towards a program logic for javascript. In POPL, pages 31-44, 2012.

[12] Google Developers. https://developers.google.com/octane/.

[13] C. S. Gordon, M. J. Parkinson, J. Parsons, A. Bromfield, and J. Duffy. Uniqueness and Reference Immutability for Safe Parallelism. In OOPSLA, 2012.

[14] C. S. Gordon, M. D. Ernst, and D. Grossman. Rely-guarantee References for Refinement Types over Aliased Mutable Data. In Proceedings of the 34th ACM SIGPLAN Conference on Programming Language Design and Implementation, PLDI '13, pages 73-84, New York, NY, USA, 2013. ACM. ISBN 978-1-4503-2014-6.

[15] A. Guha, M. Fredrikson, B. Livshits, and N. Swamy. Verified security for browser extensions. In Proceedings of the 2011 IEEE Symposium on Security and Privacy, SP '11, pages 115130, Washington, DC, USA, 2011. IEEE Computer Society.

[16] S. Guo and B. Hackett. Fast and Precise Hybrid Type Inference for JavaScript. In PLDI, 2012.
[17] C. Haack and E. Poll. Type-Based Object Immutability with Flexible Initialization. In ECOOP, Berlin, Heidelberg, 2009.

[18] A. Igarashi, B. C. Pierce, and P. Wadler. Featherweight Java: A Minimal Core Calculus for Java and GJ. ACM Trans. Program. Lang. Syst., 23(3):396-450, May 2001. ISSN 01640925.

[19] K. Knowles and C. Flanagan. Hybrid Type Checking. ACM Trans. Program. Lang. Syst., 32(2), Feb. 2010.

[20] K. Knowles and C. Flanagan. Compositional reasoning and decidable checking for dependent contract types. In Proceedings of the 3rd Workshop on Programming Languages Meets Program Verification, PLPV'09, pages 27-38, New York, NY, USA, 2008. ACM. ISBN 978-1-60558-330-3.

[21] B. S. Lerner, J. G. Politz, A. Guha, and S. Krishnamurthi. TeJaS: Retrofitting Type Systems for JavaScript. In Proceedings of the 9th Symposium on Dynamic Languages, 2013.

[22] Microsoft Corporation. TypeScript v1.4. http://www. typescriptlang. org/.

[23] F. Militão, J. Aldrich, and L. Caires. ECOOP 2014 - ObjectOriented Programming: 28th European Conference, Uppsala, Sweden, July 28 - August 1, 2014. Proceedings, chapter RelyGuarantee Protocols, pages 334-359. Springer Berlin Heidel-

[24] G. Nelson. Techniques for program verification. Technical Report CSL81-10, Xerox Palo Alto Research Center, 1981.

[25] N. Nystrom, V. Saraswat, J. Palsberg, and C. Grothoff. Constrained Types for Object-oriented Languages. In Proceedings of the 23rd ACM SIGPLAN Conference on Object-oriented Programming Systems Languages and Applications, OOPSLA '08, pages 457-474, New York, NY, USA, 2008. ACM.

[26] X. Qi and A. C. Myers. Masked types for sound object initialization. In Proceedings of the 36th Annual ACM SIGPLANSIGACT Symposium on Principles of Programming Languages, POPL '09, pages 53-65, New York, NY, USA, 2009. ACM.

[27] A. Rastogi, N. Swamy, C. Fournet, G. Bierman, and P. Vekris. Safe \& efficient gradual typing for typescript. In Proceedings of the 42Nd Annual ACM SIGPLAN-SIGACT Symposium on Principles of Programming Languages, POPL '15, pages 167-180, New York, NY, USA, 2015. ACM. ISBN 978-14503-3300-9.

[28] P. M. Rondon, M. Kawaguci, and R. Jhala. Liquid Types. In Proceedings of the ACM SIGPLAN Conference on Programming Language Design and Implementation, 2008.

[29] J. Rushby, S. Owre, and N. Shankar. Subtypes for Specifications: Predicate Subtyping in PVS. IEEE TSE, 1998.

[30] E. L. Seidel, N. Vazou, and R. Jhala. Type targeted testing. In Proceedings of the 24th European Symposium on Programming on Programming Languages and Systems - Volume 9032, pages 812-836, New York, NY, USA, 2015. SpringerVerlag New York, Inc. ISBN 978-3-662-46668-1.

[31] F. Smith, D. Walker, and G. Morrisett. Alias Types. In In European Symposium on Programming, pages 366-381. Springer-Verlag, 1999. 
[32] A. J. Summers and P. Mueller. Freedom before commitment: A lightweight type system for object initialisation. In Proceedings of the 2011 ACM International Conference on Object Oriented Programming Systems Languages and Applications, OOPSLA '11, pages 1013-1032, New York, NY, USA, 2011. ACM. ISBN 978-1-4503-0940-0.

[33] N. Swamy, J. Chen, C. Fournet, P.-Y. Strub, K. Bhargavan, and J. Yang. Secure distributed programming with valuedependent types. In Proceedings of the 16th ACM SIGPLAN International Conference on Functional Programming, ICFP '11, pages 266-278, New York, NY, USA, 2011. ACM.

[34] O. Tardieu, N. Nystrom, I. Peshansky, and V. Saraswat. Constrained kinds. In Proceedings of the ACM International Conference on Object Oriented Programming Systems Languages and Applications, OOPSLA '12, pages 811-830, New York, NY, USA, 2012. ACM.

[35] P. Thiemann. Towards a Type System for Analyzing Javascript Programs. In Proceedings of the 14th European Conference on Programming Languages and Systems, 2005.

[36] S. Tobin-Hochstadt and M. Felleisen. Logical Types for Untyped Languages. In Proceedings of the 15th ACM SIGPLAN International Conference on Functional Programming, 2010.

[37] N. Vazou, E. L. Seidel, R. Jhala, D. Vytiniotis, and S. PeytonJones. Refinement Types for Haskell. In Proceedings of the 19th ACM SIGPLAN International Conference on Functional Programming, 2014.

[38] P. Vekris, B. Cosman, and R. Jhala. Trust, but verify: Twophase typing for dynamic languages. In 29th European Conference on Object-Oriented Programming, ECOOP 2015, July 5-10, 2015, Prague, Czech Republic, pages 52-75, 2015.

[39] H. Xi and F. Pfenning. Dependent Types in Practical Programming. In Proceedings of the 26th ACM SIGPLAN-SIGACT Symposium on Principles of Programming Languages, 1999.

[40] B. Yankov. http://definitelytyped.org.

[41] Y. Zibin, A. Potanin, M. Ali, S. Artzi, A. Kiezun, and M. D. Ernst. Object and Reference Immutability Using Java Generics. In Proceedings of the the 6th Joint Meeting of the European Software Engineering Conference and the ACM SIGSOFT Symposium on The Foundations of Software Engineering, 2007.

[42] Y. Zibin, A. Potanin, P. Li, M. Ali, and M. D. Ernst. Ownership and Immutability in Generic Java. In OOPSLA, 2010.

[43] Y. Zibin, D. Cunningham, I. Peshansky, and V. Saraswat. Object initialization in $\mathrm{x} 10$. In Proceedings of the 26th European Conference on Object-Oriented Programming, ECOOP'12, pages 207-231, Berlin, Heidelberg, 2012. Springer-Verlag. 


\section{A. Full System}

In this section we present the full type system for the core language of $\S 3$ of the main paper.

\section{A.1 Formal Languages}

FRSC Figure 9 shows the full syntax for the input language. The type language is the same as described in the main paper. The operational semantics, shown in Figure 10, is borrowed from Safe TypeScript [27], with certain simplifications since the language we are dealing with is simpler than the one used there. We use evaluation contexts $\mathrm{E}$, with a left to right evaluation order.

\begin{tabular}{|c|c|c|c|}
\hline \multirow[b]{2}{*}{ Expressions } & \multicolumn{3}{|r|}{ Syntax } \\
\hline & $\mathrm{e}$ & $::=$ & $\mathrm{x}|\mathrm{c}|$ this $\mid$ e.f $\mid$ e.m $(\overline{\mathrm{e}}) \mid$ new $C(\overline{\mathrm{e}}) \mid<T>\mathrm{e}$ \\
\hline Statements & $\mathrm{s}$ & $::=$ & $\operatorname{var} x=e|e . f=e| x=e \mid \operatorname{if}(e)\{s\}$ else $\{s\}|s ; s|$ skip \\
\hline Field Decl. & $\mathrm{F}$ & $::=$ & $\cdot|\circ \mathrm{f}: T| \square \mathrm{f}: T \mid \mathrm{F}_{1} ; \mathrm{F}_{2}$ \\
\hline Method Body & B & $::=$ & s; returne \\
\hline Expr. or Body & w & $::=$ & e $\mid B$ \\
\hline Method Decl. & M & $::=$ & $\cdot|\mathrm{m}(\overline{\mathrm{x}}: \bar{T})\{p\}: T| \mathrm{M}_{1} ; \mathrm{M}_{2}$ \\
\hline Field Def. & $\widetilde{F}$ & $::=$ & $\cdot|\mathrm{f}:=\mathrm{v}| \widetilde{\mathrm{F}}_{1} ; \widetilde{\mathrm{F}}_{2}$ \\
\hline Method Def. & $\widetilde{\mathrm{M}}$ & $::=$ & $\cdot|\mathrm{m}(\overline{\mathrm{x}}: \bar{T})\{p\}: T\{\mathrm{~B}\}| \widetilde{\mathrm{M}}_{1} ; \widetilde{\mathrm{M}}_{2}$ \\
\hline Class Def. & $\widetilde{C}$ & $::=$ & class $C\{p\}$ extends $R\{\mathrm{~F}, \widetilde{\mathrm{M}}\}$ \\
\hline Signature & $\mathrm{S}$ & $::=$ & $\cdot|\widetilde{\mathrm{C}}| \mathrm{S}_{1} ; \mathrm{S}_{2}$ \\
\hline Program & $\mathrm{P}$ & $::=$ & $\mathrm{S} ; \mathrm{B}$ \\
\hline
\end{tabular}

Runtime Configuration

$$
\begin{aligned}
& \text { Evaluation Context } \quad \mathrm{E} \quad::=\text { [] } \mid \text { E.f }|\mathrm{E} . \mathrm{m}(\overline{\mathrm{e}})| \mathrm{v} \cdot \mathrm{m}(\overline{\mathrm{v}}, \mathrm{E}, \overline{\mathrm{e}}) \mid \text { new } C(\overline{\mathrm{v}}, \mathrm{E}, \overline{\mathrm{e}})|<T>\mathrm{E}| \operatorname{var} \mathrm{x}=\mathrm{E} \mid \\
& \text { E. } f=e \mid \text { v. } f=E|x=E| \text { if }(E)\{s\} \text { else }\{s\} \mid \text { return E }|E ; s| E ; \text { return e } \\
& \text { Runtime Conf. } \quad \mathrm{R} \quad::=\mathrm{K} ; \mathrm{s} \\
& \text { State } \quad \mathrm{K} \quad::=\mathrm{S} ; \mathrm{L} ; \mathrm{X} ; \mathrm{H} \\
& \text { Store } \quad \mathrm{L} \quad::=\cdot|\mathrm{x} \mapsto \mathrm{v}| \mathrm{L}_{1} ; \mathrm{L}_{2} \\
& \text { Value } \quad \mathrm{v}::=1 \mid \mathrm{c} \\
& \text { Stack } \quad \mathrm{X} \quad::=\cdot \mid \mathrm{X} ; \mathrm{L}, \mathrm{E} \\
& \text { Heap } \quad \mathrm{H} \quad::=\cdot|\mathrm{l} \mapsto \mathrm{O}| \mathrm{H}_{1} ; \mathrm{H}_{2} \\
& \text { Object } \quad 0 \quad::=\{\text { proto: } 1 ; f: \widetilde{\mathrm{F}}\} \mid\{\text { name: } C ; \text { proto: } 1 ; \mathrm{m}: \widetilde{\mathrm{M}}\}
\end{aligned}
$$$$
\text { Figure 9: FRSC: syntax and runtime configuration }
$$ 


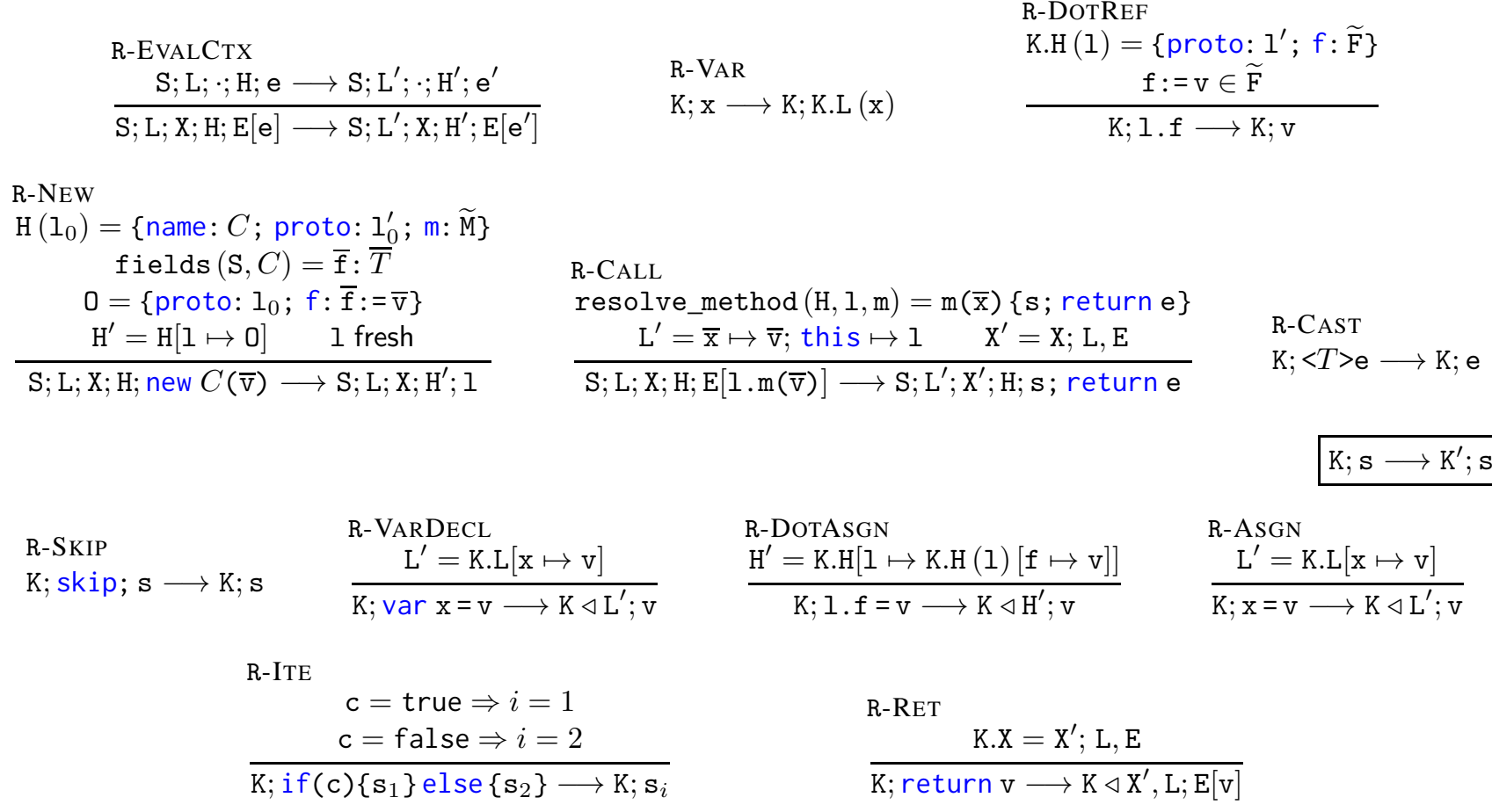

Figure 10: Reduction Rules for FRSC (adapted from Safe TypeScript [27])

IRSC Figure 11 shows the full syntax for the SSA transformed language. The reduction rules of the operational semantics for language IRSC are shown in Figure 12. We use evaluation contexts $E$, with a left to right evaluation order.

\section{A.2 SSA Transformation}

Section 3 of the main paper describes the SSA transformation from FRSC to IRSC. This section provides more details and extends the transformation to runtime configurations, to enable the statement and proof of our consistency theorem.

\section{A.2.1 Static Tranformation}

Figure 13 includes some additional transformation rules that supplement the rules of Figure 3 of the main paper. The main program transformation judgment is:

$$
\mathrm{P} \hookrightarrow P \rightsquigarrow \Delta
$$

A global SSA enviornment $\Delta$ is the result of the translation of the entire program $\mathrm{P}$ to $P$. In particular, in a program translation tree:

- each expression node introduces a single binding to the relevant SSA environment

$$
\delta \Vdash \mathrm{e} \hookrightarrow e \quad \text { produces binding } \quad \mathrm{e} \mapsto \delta
$$

- each statement introduces two bindings, one for the input environment and one for the output (we use the notation $\lceil\cdot\rceil$ and $\lfloor\cdot\rfloor$, respectively):

$$
\delta_{0} \Vdash \mathbf{s} \hookrightarrow u ; \delta_{1} \quad \text { produces bindings } \quad\lceil\mathbf{s}\rceil \mapsto \delta_{0} \quad\lfloor\mathbf{s}\rfloor \mapsto \delta_{1}
$$

We assume all AST nodes are uniquely identified.

\section{A.2.2 Runtime Configuration Tranformation}

Figure 14 includes the rules for translating runtime configurations. The main judgment is of the form:

$$
\mathrm{K} ; \mathrm{w} \stackrel{\Delta}{\hookrightarrow} K ; e
$$




\section{Syntax}

\begin{tabular}{|c|c|c|c|}
\hline Expression & $e$ & $::=$ & $x|c|$ this $\mid$ e.f $\mid$ e.m $(\bar{e}) \mid$ new $C(\bar{e}) \mid e$ as $T\left|e_{1} . f \leftarrow e_{2}\right| u\langle e\rangle$ \\
\hline SSA context & $u$ & $::=$ & \langle\rangle $\mid$ let $x=e$ in \langle\rangle $\mid$ letif $[\bar{\phi}](e) ? u_{1}: u_{2}$ in \langle\rangle \\
\hline Term & $w$ & $::=$ & $e \mid u$ \\
\hline$\Phi$-Vars & $\phi$ & $::=$ & $\left(x, x_{1}, x_{2}\right)$ \\
\hline Field Decl. & $F$ & $::=$ & $\cdot|\circ f: T| \square f: T \mid F_{1} ; F_{2}$ \\
\hline Method Decl. & $M$ & $::=$ & $\cdot|m(\bar{x}: \bar{T})\{p\}: T| M_{1} ; M_{2}$ \\
\hline Field Def. & $\widetilde{F}$ & $::=$ & $\cdot|f:=v| \widetilde{F}_{1} ; \widetilde{F}_{2}$ \\
\hline Method Def. & $\widetilde{M}$ & $::=$ & $\cdot|\operatorname{def} m(\bar{x}: \bar{T})\{p\}: T=e| \widetilde{M}_{1} ; \widetilde{M}_{2}$ \\
\hline Class Def. & $\widetilde{C}$ & $::=$ & class $C\{p\} \triangleleft R\{F ; \widetilde{M}\}$ \\
\hline Signature & $S$ & $::=$ & $\cdot|\widetilde{C}| S_{1} ; S_{2}$ \\
\hline Program & $P$ & $::=$ & $S ; e$ \\
\hline
\end{tabular}

Runtime Configuration

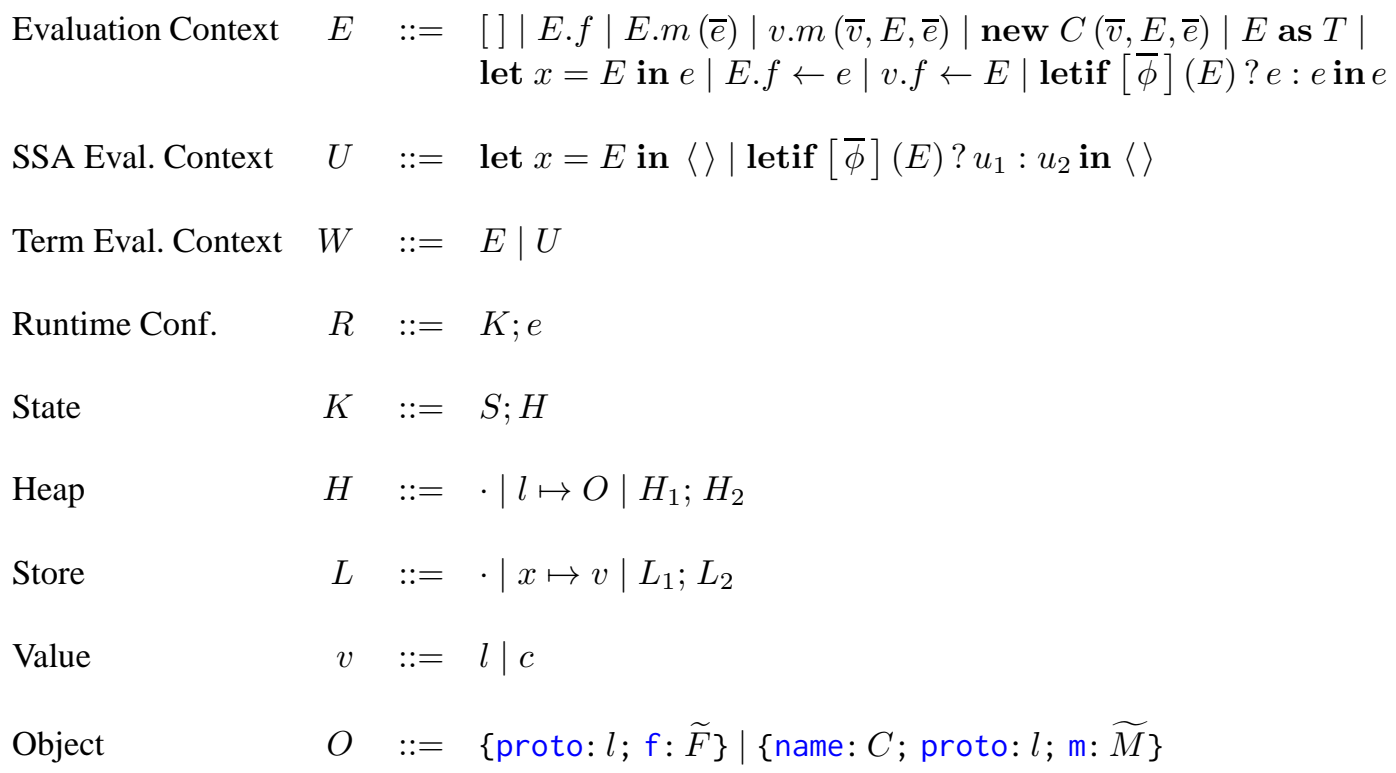

Figure 11: IRSC: syntax and runtime configuration

This assumes that the program containing expression (or body) w was SSA-translated producing a global SSA environment $\Delta$. Rule S-EXP-RTCONF translates a term w under a state K. This process gets factored into the translation of:

- the signatures K.S, which is straight-forward (same as in static translation),

- the heap K.H, which is described in Figure 15, and 


$$
\begin{aligned}
& \text { R-FIELD } \\
& \text { RC-ECTX } \left.\quad K . H(l)=\text { \{proto: } l^{\prime} ; f: \widetilde{F}\right\} \\
& \frac{K ; e \longrightarrow K^{\prime} ; e^{\prime}}{K ; E[e] \longrightarrow K^{\prime} ; E\left[e^{\prime}\right]} \quad \frac{f:=v \in \widetilde{F}}{K ; l . f \longrightarrow K ; v} \\
& \text { R-CALL } \\
& \text { resolveMethod }(H, l, m)=(\operatorname{def} m(\bar{x}: \bar{S})\{p\}: T=e) \\
& \text { eval }([\bar{v} / \bar{x}, l / \text { this }] p)=\text { true } \\
& K ; l . m(\bar{v}) \longrightarrow K ;[\bar{v} / \bar{x}, l / \text { this }] e \\
& \text { R-NEW } \\
& \mathrm{H}\left(l_{0}\right)=\left\{\text { name: } C \text {; proto: } l_{0}^{\prime} ; \mathrm{m}: \widetilde{M}\right\} \\
& \text { fields }(S, C)=\bar{f}: \bar{T} \\
& \text { R-CAST } \\
& \frac{\Gamma \vdash K(l): S ; S \leq T}{K ; l \text { as } T \longrightarrow K ; l} \\
& O=\left\{\text { proto: } l_{0} ; \mathrm{f}: \bar{f}:=\bar{v}\right\} \\
& \frac{\begin{array}{l}
\text { R-DotAsGn } \\
H^{\prime}=K . H[l \mapsto K . H(l)[f \mapsto v]]
\end{array}}{K ; l . f \leftarrow v \longrightarrow K \triangleleft H^{\prime} ; v} \\
& H^{\prime}=H[l \mapsto O] \quad l \text { fresh } \\
& \text { R-LETIN } \\
& K ; \text { let } x=v \text { in } e \longrightarrow K ;[v / x] e \\
& S ; H ; \text { new } C(\bar{v}) \longrightarrow S ; H^{\prime} ; l \\
& \text { R-LETIF } \\
& \frac{c=\text { true } \Rightarrow i=1 \quad c=\text { false } \Rightarrow i=2}{K ; \text { letif }\left[\bar{x}, \bar{x}_{1}, \bar{x}_{2}\right](c) ? u_{1}: u_{2} \text { in } e \longrightarrow K ; u_{i}\left\langle\left[\overline{x_{i}} / \bar{x}\right] e\right\rangle}
\end{aligned}
$$

Figure 12: Reduction Rules for IRSC

\section{SSA Transformation}

$\mathrm{P} \hookrightarrow P \rightsquigarrow \Delta$

Program Translation

$$
\begin{gathered}
\mathrm{S} \hookrightarrow S \text { produces } \Delta_{1} \\
\cdot \Vdash \mathrm{B} \hookrightarrow e \text { produces } \Delta_{2} \\
\frac{\mathrm{S} ; \mathrm{B} \hookrightarrow S ; e \rightsquigarrow \Delta_{1} \cup \Delta_{2}}{}
\end{gathered}
$$

$\mathrm{S} \hookrightarrow S$

Signature Translation

$$
\begin{aligned}
& \begin{array}{llrl}
\text { S-SIGS-EMP } & \text { S-SIGS-BND } & \widetilde{\mathrm{M}} \hookrightarrow \widetilde{M} & \widetilde{\mathrm{F}} \hookrightarrow F \\
. \hookrightarrow \cdot & &
\end{array} \\
& \overline{\text { class } C\{p\} \text { extends } R\{\mathrm{~F}, \widetilde{\mathrm{M}}\} \hookrightarrow \operatorname{class} C\{p\} \triangleleft R\{F ; \widetilde{M}\}} \quad \frac{\quad}{\delta \Vdash \mathrm{S}_{1} ; \mathrm{S}_{2} \hookrightarrow S_{1} ; S_{2}} \\
& \delta \Vdash \mathrm{e} \hookrightarrow e \quad \delta \Vdash \mathrm{s} \hookrightarrow u ; \delta^{\prime} \\
& \text { S-CONST } \\
& \mathrm{c} \hookrightarrow \text { toValue (c) } \\
& \text { S-CALL } \\
& \delta \Vdash \mathrm{e} \hookrightarrow e \quad \delta \Vdash \mathrm{e}_{i} \hookrightarrow \bar{e}_{i} \\
& \frac{\text { toString }(\mathrm{m})=\text { toString }(m) \quad m \text { fresh }}{\delta \Vdash \text { e.m( }\left(\overline{\mathrm{e}}_{i}\right) \hookrightarrow e . m\left(\bar{e}_{i}\right)}
\end{aligned}
$$

Expression and Statement Translations (selected)

Figure 13: Additional SSA Transformation Rules

- term w under a local store K.L and a stack K.X.

The last part breaks down into rules that expose the structure of the stack. Rule S-STACK-EMP translates configurations involving an empty stack, which are delegated to the judgment L; $\stackrel{\mathrm{H}, \Delta}{\longrightarrow} e$, and rule S-STACK-CONS separately translates the top of the stack and the rest of the stack frames, and then composes them into a single target expression.

Finally, judgments of the forms L; X; w $\stackrel{\mathrm{H}, \Delta}{\longrightarrow} e$ and L; X; E $\stackrel{\mathrm{H}, \Delta}{\longrightarrow} W$ translate expressions and statements under a local store $\mathrm{L}$. The rules here are similar to their static counterparts. The key difference stems from the fact that in IRSC variable are replaced with the respective values as soon as they come into scope. On the contrary, in FRSC variables are only instantiated with the matching (in the store) value when they get into an evaluation position. To wit, rule SR-VARREF performs the necessary 
substitution $\theta$ on the translated variable, which we calculate though the meta-function toSubst, defined as follows:

$$
\operatorname{toSubst}(\delta, \mathrm{L}, \mathrm{H}) \doteq \begin{cases}\{[v / x] \mid \mathrm{x} \mapsto x \in \delta, \mathrm{x} \mapsto \mathrm{v} \in \mathrm{L}, \mathrm{H} ; \mathrm{v} \hookrightarrow v\} & \text { if } \operatorname{dom}(\delta)=\operatorname{dom}(\mathrm{L}) \\ \text { impossible } & \text { otherwise }\end{cases}
$$

\section{A.3 Object Constraint System}

Our system leverages the idea introduced in the formall core of X10 [25] to extend a base constraint system $\mathscr{C}$ with a larger constraint system $\mathscr{O}(\mathscr{C})$, built on top of $\mathscr{C}$. The original system $\mathscr{C}$ comprises formulas taken from a decidable SMT logic [24], including, for example, linear arithmetic constraints and uninterpreted predicates. The Object Constraint System $\mathscr{O}(\mathscr{C})$ introduces the constraints:

- class $(C)$, which it true for all classes $C$ defined in the program;

- $x$ haslmm $\mathrm{f}$, to denote that the immutable field $\mathrm{f}$ is accessible from variable $x$;

- $x$ hasMut $\mathrm{f}$, to denote that the mutable field $\mathrm{f}$ is accessible from variable $x$; and

- fields $(x)=\mathrm{F}$, to expose all fields available to $x$.

Figure 16 shows the constraint system as ported from CFG [25]. We refer the reader to that work for details. The main differences are syntactic changes to account for our notion of strengthening. Also the SC-FIELD rule accounts now for both immutable and mutable fields. The main judgment here is of the form:

$$
\Gamma \vdash \vdash_{S} p
$$

where $S$ is the set of classes defined in the program. Substitutions and strengthening operations on field declarations are performed on the types of the declared fields (e.g. SC-FIELD-I, SC-FIELD-C).

\section{A.4 Well-formedness Constraints}

The well-formedness rules for predicates, terms, types and heaps can be found in Figure 17. The majority of these rules are routine.

The judgment for term well-formedness assigns a sort to each term $t$, which can be thought of as a base type. The judgment $\Gamma \vdash_{q} \bar{t}$ is used as a shortcut for any further constraints that the $f$ operator might impose on its arguments $\bar{t}$. For example if $f$ is the equality operator then the two arguments are required to have types that are related via subtyping, i.e. if $t_{1}: N_{1}$ and $t_{2}: N_{2}$, it needs to be the case that $N_{1} \leq N_{2}$ or $N_{2} \leq N_{1}$.

Type well-formedness is typical among similar refinement types [20].

\section{A.5 Subtyping}

Figure 18 presents the full set of sybtyping rules, which borrows ideas from similar systems [20, 28]. 


\section{SSA Transformation for Runtime Configurations}

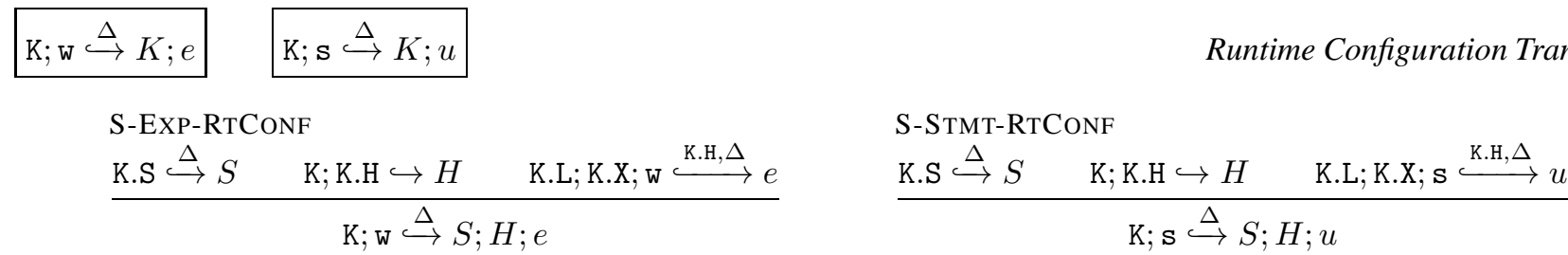

\begin{tabular}{|c|c|c|c|c|c|}
\hline $\mathrm{L} ; \mathrm{X} ; \mathrm{w} \stackrel{\mathrm{H}, \Delta}{\longrightarrow} e$ & $\mathrm{~L} ; \mathrm{X} ; \mathrm{E} \stackrel{\mathrm{H}, \Delta}{\longrightarrow} W$ & & & \multicolumn{2}{|c|}{ Runtime Stack Translatio } \\
\hline $\begin{array}{l}\text { S-STACK-EMP } \\
\mathrm{L} ; \mathrm{w} \stackrel{\mathrm{H}, \Delta}{\longrightarrow} e\end{array}$ & $\begin{array}{l}\text { S-EC-STACK-EMP } \\
\mathrm{L} ; \mathrm{E} \stackrel{\mathrm{H}, \Delta}{\longrightarrow} W\end{array}$ & $\begin{array}{l}\text { S-STACK-CONS } \\
\mathrm{L}_{0} ; \cdot ; \text { w } \stackrel{\mathrm{H}, \Delta}{\longrightarrow} e_{0}\end{array}$ & $\mathrm{~L} ; \mathrm{X} ; \mathrm{E} \stackrel{\mathrm{H}, \Delta}{\longrightarrow} E$ & $\begin{array}{l}\text { S-EC-STACK-CONS } \\
\mathrm{L}_{0} ; \cdot ; \mathrm{E}_{0} \stackrel{\mathrm{H}, \Delta}{\longrightarrow} W_{0}\end{array}$ & $\mathrm{~L} ; \mathrm{X} ; \mathrm{E} \stackrel{\mathrm{H}, \Delta}{\longrightarrow} E$ \\
\hline$\overline{\mathrm{L} ; \cdot ; \mathrm{w} \stackrel{\mathrm{H}, \Delta}{\longrightarrow} e}$ & $\overline{\mathrm{L} ; \cdot ; \mathrm{E} \stackrel{\mathrm{H}, \Delta}{\longrightarrow} W}$ & $\mathrm{~L}_{0} ;(\mathrm{X} ; \mathrm{L}, \mathrm{E})$ & $\stackrel{\mathrm{H}, \Delta}{\longrightarrow} E\left[e_{0}\right]$ & $\mathrm{L}_{0} ;(\mathrm{X} ; \mathrm{L}, \mathrm{E}) ; \mathrm{E}_{0}$ & $\stackrel{\mathrm{H}, \Delta}{\longrightarrow} E\left[W_{0}\right]$ \\
\hline
\end{tabular}

$\widetilde{\mathrm{M}} \stackrel{\Delta}{\hookrightarrow} \widetilde{M} \quad \mathrm{~L} ; \mathrm{w} \stackrel{\mathrm{H}, \Delta}{\longrightarrow} e \quad \mathrm{~L} ; \mathrm{s} \stackrel{\mathrm{H}, \Delta}{\longrightarrow} u$

Runtime Term Translation (selected rules)

\begin{tabular}{|c|c|c|c|}
\hline $\begin{array}{l}\text { SR-METH } \\
\qquad ; \mathrm{B} \stackrel{\cdot, \Delta}{\longrightarrow} e\end{array}$ & $\begin{array}{l}\mathrm{SR}-\mathrm{VAL} \\
\mathrm{H} ; \mathrm{v} \hookrightarrow v\end{array}$ & $\begin{array}{l}\text { SR-VARREF } \\
\quad \Delta(\mathrm{x}) \Vdash \mathrm{x} \hookrightarrow x \\
\theta=\operatorname{toSubst}(\Delta(\mathrm{x}), \mathrm{L}, \mathrm{H})\end{array}$ & $\begin{array}{l}\text { SR-CALL } \\
\mathrm{L} ; \mathrm{e} \underset{\mathrm{H}, \Delta}{\longrightarrow} e \quad \text { L; } ; \overline{\mathrm{e}} \stackrel{\mathrm{H}, \Delta}{\longrightarrow} \bar{e} \\
\text { toString }(\mathrm{m})=\text { toString }(m)\end{array}$ \\
\hline $\mathrm{m}(\overline{\mathrm{x}})\{\mathrm{B}\} \stackrel{\Delta}{\hookrightarrow} \operatorname{def} m(\bar{x})=e$ & $\mathrm{~L} ; \mathrm{v} \stackrel{\mathrm{H}, \Delta}{\longrightarrow} v$ & $\mathrm{~L} ; \mathrm{x} \stackrel{\mathrm{H}, \Delta}{\longrightarrow} \theta x$ & $\mathrm{~L} ; \mathrm{e} \cdot \mathrm{m}(\overline{\mathrm{e}}) \stackrel{\mathrm{H}, \Delta}{\longrightarrow} e \cdot m(\bar{e})$ \\
\hline
\end{tabular}

SR-BODY

$$
\frac{\mathrm{L} ; \mathrm{s} \stackrel{\mathrm{H}, \Delta}{\longrightarrow} u \quad \Delta^{\prime}=\Delta[\mathrm{e} \mapsto \Delta\lfloor\mathrm{s}\rfloor] \quad \mathrm{L} ; \mathrm{e} \stackrel{\mathrm{H}, \Delta^{\prime}}{\longrightarrow} e}{\mathrm{~L} ; \mathrm{s} ; \text { return e } \stackrel{\mathrm{H}, \Delta}{\longrightarrow} u\langle e\rangle}
$$

SR-ITE

$$
\begin{gathered}
\text { L; } \mathrm{e} \stackrel{\mathrm{H}, \Delta}{\longrightarrow} e \quad \mathrm{~L} ; \mathbf{s}_{1} \stackrel{\mathrm{H}, \Delta}{\longrightarrow} u_{1} \quad \text { L; } \mathbf{s}_{2} \stackrel{\mathrm{H}, \Delta}{\longrightarrow} u_{2} \\
\frac{\left(\overline{\mathrm{x}}, \bar{x}_{1}, \bar{x}_{2}\right)=\Delta\left\lfloor\mathbf{s}_{1}\right\rfloor \bowtie \Delta\left\lfloor\mathbf{s}_{2}\right\rfloor \quad \bar{x}=\Delta\left\lfloor\text { if }(\mathrm{e})\left\{\mathbf{s}_{1}\right\} \text { else }\left\{\mathbf{s}_{2}\right\}\right\rfloor(\overline{\mathrm{x}})}{\mathrm{L} ; \text { if }(\mathrm{e})\left\{\mathbf{s}_{1}\right\} \text { else }\left\{\mathbf{s}_{2}\right\} \stackrel{\mathrm{H}, \Delta}{\longrightarrow} \text { letif }\left[\bar{x}, \bar{x}_{1}, \bar{x}_{2}\right](e) ? u_{1}: u_{2} \text { in }\langle\rangle}
\end{gathered}
$$

SR-VARDECL

$$
\frac{\mathrm{x} \mapsto x \in \Delta\lfloor\operatorname{var} \mathrm{x}=\mathrm{e}\rfloor \quad \mathrm{L} ; \mathrm{e} \stackrel{\mathrm{H}, \Delta}{\longrightarrow} e}{\mathrm{~L} ; \operatorname{var} \mathrm{x}=\mathrm{e} \stackrel{\mathrm{H}, \Delta}{\longrightarrow} \text { let } x=e \text { in }\langle\rangle}
$$

$\mathrm{L} ; \mathrm{E} \stackrel{\mathrm{H}, \Delta}{\longrightarrow} W$

$$
\begin{aligned}
& \mathrm{L} ; \mathrm{E} \stackrel{\mathrm{H}, \Delta}{\longrightarrow} E \quad f \text { fresh } \\
& \mathrm{L} ;[] \stackrel{\mathrm{H}, \Delta}{\longrightarrow}[] \\
& \frac{\text { toString }(\mathrm{f})=\text { toString }(f)}{\text { L; E.f } \stackrel{\mathrm{H}, \Delta}{\longrightarrow} E . f} \\
& \frac{\mathrm{L} ; \cdot ; \mathrm{x} \stackrel{\mathrm{H}, \Delta}{\longrightarrow} x \quad \mathrm{~L} ; \mathrm{E} \stackrel{\mathrm{H}, \Delta}{\longrightarrow} E}{\mathrm{~L} ; \operatorname{var} \mathrm{x}=\mathrm{E} \stackrel{\mathrm{H}, \Delta}{\longrightarrow} \text { let } x=E \text { in }\langle\rangle}
\end{aligned}
$$

$\frac{\mathrm{SR}-A S G N}{\mathrm{x} \mapsto x \in \Delta\lfloor\mathrm{x}=\mathrm{e}\rfloor \quad \mathrm{L} ; \mathrm{e} \stackrel{\mathrm{H}, \Delta}{\longrightarrow} e}$
$\mathrm{~L} ; \mathrm{x}=\mathrm{e} \stackrel{\mathrm{H}, \Delta}{\longrightarrow}$ let $x=e$ in \langle\rangle

Evaluation Context Translation (selected rules)

$$
\begin{gathered}
\mathrm{L} ; \mathrm{E} \stackrel{\mathrm{H}, \Delta}{\longrightarrow} E \quad m \text { fresh } \\
\frac{\text { toString }(\mathrm{m})=\text { toString }(m) \quad \mathrm{L} ; \cdot ; \overline{\mathrm{e}} \stackrel{\mathrm{H}, \Delta}{\longrightarrow} \bar{e}}{\mathrm{~L} ; \mathrm{E} . \mathrm{m}(\overline{\mathrm{e}}) \stackrel{\mathrm{H}, \Delta}{\longrightarrow} E \cdot m(\bar{e})} \\
\frac{\mathrm{L} ; \mathrm{E} \stackrel{\mathrm{H}, \Delta}{\longrightarrow} U \quad \mathrm{~L} ; \mathrm{s} \stackrel{\mathrm{H}, \Delta}{\longrightarrow} u}{\mathrm{~L} ; \mathrm{E} ; \mathrm{s} \stackrel{\mathrm{H}, \Delta}{\longrightarrow} U\langle u\rangle}
\end{gathered}
$$

Figure 14: SSA Transformation Rules for Runtime Configurations 
$\mathrm{K} ; \mathrm{H} \hookrightarrow H \quad \mathrm{H} ; \mathrm{v} \hookrightarrow v$

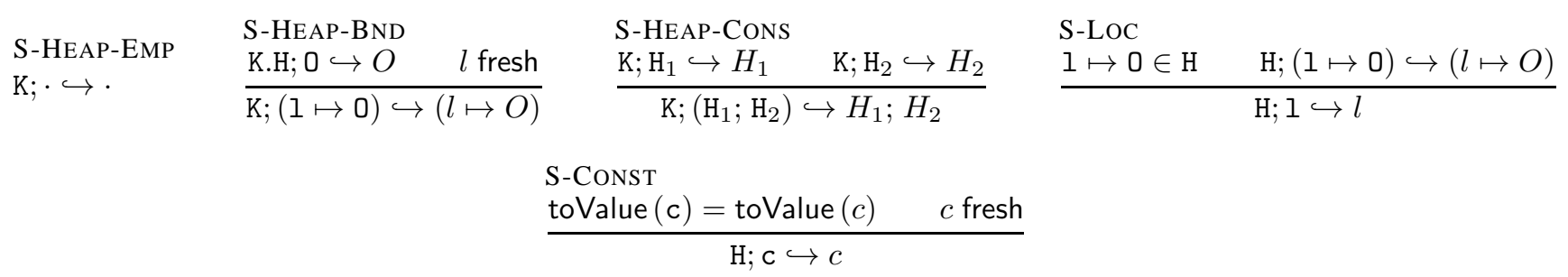

$\mathrm{H} ; \mathrm{O} \hookrightarrow \mathrm{O}$

Heap Object Translation

$$
\frac{\mathrm{H} ; 1 \hookrightarrow l \quad \mathrm{H} ; \mathrm{F} \hookrightarrow \widetilde{F}}{\mathrm{H} ;\{\text { proto: } 1 ; \mathrm{f}: \widetilde{\mathrm{F}}\} \hookrightarrow\{\text { proto: } l ; \mathrm{f}: \widetilde{F}\}} \quad \frac{\mathrm{H} ; 1 \hookrightarrow l \quad \widetilde{\mathrm{M}} \hookrightarrow \widetilde{M}}{\mathrm{H} ;\{\text { name: } C ; \text { proto: } 1 ; \mathrm{m}: \widetilde{\mathrm{M}}\} \hookrightarrow\{\text { name: } C ; \operatorname{proto}: l ; \mathrm{m}: \widetilde{M}\}}
$$

Figure 15: SSA Transformation Rules for Heaps and Objects

\section{Structural Constraints}

SC-Class
$\frac{\text { class } C\{p\} \triangleleft R\{F ; \widetilde{M}\} \in S}{\Gamma \vdash_{S} \operatorname{class}(C)}$

$$
\begin{aligned}
& \text { SC-INv } \\
& \frac{\Gamma \vdash_{S} x: C, \text { class }(C)}{\Gamma \vdash_{S} \operatorname{inv}(C, x)}
\end{aligned}
$$

\section{SC-FIELD-I}

SC-OBJECT
$x:$ Object $\vdash_{S}$ fields $(x) \varnothing$

$$
\Gamma, x: D \vdash_{S} \text { fields }(x)=F
$$

class $C\{p\} \triangleleft R\left\{F^{\prime} ; \widetilde{M}\right\} \in S$

\section{SC-FIELD}

$\frac{\Gamma \vdash_{S} \text { fields }(x)=\circ \overline{f_{i}}: \overline{T_{i}}, \square \overline{g_{i}}: \overline{S_{i}}}{\Gamma \vdash_{S} x \text { haslmm } f_{i}: T_{i}}$

$\Gamma \vdash_{S} x$ hasMut $g_{i}: S_{i}$
SC-METH-B

$$
\Gamma \vdash_{S} \text { class }(C) \quad \theta=[x / \text { this }]
$$$$
\text { def } m(\bar{x}: \bar{T})\{p\}: T=e \in C
$$

$\overline{\Gamma, x: C \vdash_{S} x \text { has }(\operatorname{def} m(\bar{x}: \overline{\theta T})\{\theta p\}: \theta T=e)}$

\section{SC-FIELD-C}

$\frac{\Gamma, x: C \vdash_{S} \text { fields }(x)=F}{\Gamma, x:\{\nu: C \mid p\} \vdash_{S} \text { fields }(x)=F \oplus p[x / \nu]}$

$$
\begin{aligned}
& \text { SC-Meth-C } \\
& \Gamma, x: C \vdash_{S} x \text { has }\left(\operatorname{def} m(\bar{x}: \bar{T})\left\{p_{0}\right\}: T=e\right) \\
& \Gamma, x:\{\nu: C \mid p\} \vdash_{S} x \text { has }\left(\operatorname{def} m(\bar{x}: \bar{T})\left\{p_{0}\right\}: T \text { 丹 }[x / \text { this }] p=e\right)
\end{aligned}
$$

SC-METH-I

$\Gamma, x: D \vdash_{S} x$ has $(\operatorname{def} m(\bar{x}: \bar{T})\{p\}: T=e)$ $\frac{\text { class } C\{p\} \triangleleft D\{F ; \widetilde{M}\} \in S \quad m \notin \widetilde{M}}{\Gamma, x: C \vdash_{S} x \text { has }(\operatorname{def} m(\bar{x}: \bar{T})\{p\}: T=e)}$

Figure 16: Structural Constraints (adapted from [25]) 


\begin{tabular}{|c|c|c|c|}
\hline $\begin{array}{l}\text { WP-AND } \\
\Gamma \vdash p_{1}\end{array}$ & $\Gamma \vdash p_{2}$ & $\begin{array}{l}\text { WP-NOT } \\
\Gamma \vdash p\end{array}$ & $\begin{array}{l}\text { WP-TERM } \\
\Gamma \vdash t \text { : bool }\end{array}$ \\
\hline$\overline{\Gamma \vdash p_{1}}$ & $p_{2}$ & $\Gamma \vdash \neg p$ & $\overline{\Gamma \vdash t}$ \\
\hline
\end{tabular}

\section{Well-Formed Terms}

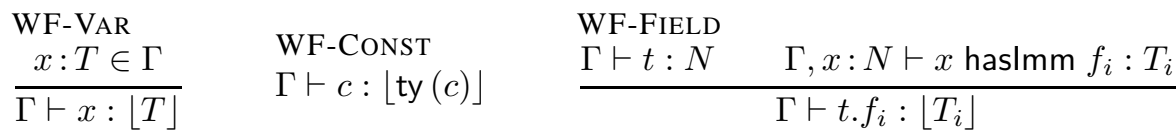

$\frac{\text { WF-FUN }}{\Gamma \vdash f: \bar{N} \rightarrow N^{\prime} \quad \Gamma \vdash_{q} \bar{t}}$

Well-Formed Types

$$
\begin{array}{ll}
\text { WT-BASE } & \text { WT-EXISTS } \\
\frac{\Gamma, \nu: N \vdash p}{\Gamma \vdash\{\nu: N \mid p\}} & \frac{\Gamma \vdash T_{1} \quad \Gamma, x: T_{1} \vdash T_{2}}{\Gamma \vdash \exists x: T_{1} \cdot T_{2}}
\end{array}
$$

\section{Well-Formed Heaps}

$$
\begin{aligned}
& \text { WF-HEAP-INST } \\
& O \doteq\left\{\text { proto: } l^{\prime} ; \mathrm{f}: \widetilde{F}\right\} \quad \widetilde{F} \doteq \circ \bar{f}:=\bar{v}_{\mathrm{I}}, \square \bar{g}:=\bar{v}_{\mathrm{M}} \quad\lfloor\Sigma(l)\rfloor=C \\
& \Gamma, z: C \vdash \text { fields }(z)=\circ \bar{f}: \bar{R}, \square \bar{g}: \bar{U} \quad \Sigma \vdash \bar{v}_{\mathrm{I}}: \bar{T}_{\mathrm{I}} \quad \Sigma \vdash \bar{v}_{\mathrm{M}}: \bar{T}_{\mathrm{M}} \quad \text { WF-HeAP-CoNS }
\end{aligned}
$$

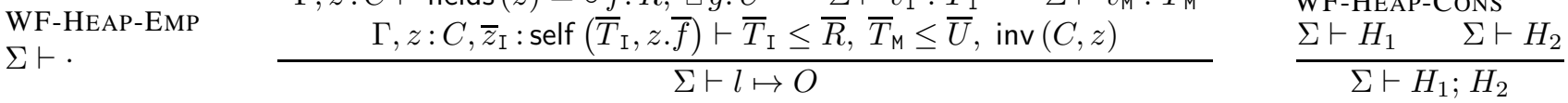

Figure 17: Well-Formedness Rules

\section{Subtyping}

$$
\begin{aligned}
& \begin{array}{lllc}
\leq \text {-REFL } & \text { S-TRANS } & \text { <-EXTENDS } & \text { <-BASE } \\
\Gamma \vdash T \leq T & \frac{\Gamma \vdash T_{1} \leq T_{2} \quad \Gamma \vdash T_{2} \leq T_{3}}{\Gamma \vdash T_{1} \leq T_{3}} & \frac{\text { class } C\{p\} \triangleleft D\{F ; \widetilde{M}\}}{\Gamma \vdash C \leq D} & \frac{\operatorname{Valid}\left(\llbracket \Gamma \rrbracket \Rightarrow \llbracket p \rrbracket \Rightarrow \llbracket p^{\prime} \rrbracket\right)}{\Gamma \vdash\{\nu: N \mid p\} \leq\left\{\nu: N^{\prime} \mid p^{\prime}\right\}}
\end{array} \\
& \begin{array}{lll}
\text { S-WITNESS } & \text { <-BIND } \\
\frac{\Gamma \vdash e: S}{\Gamma \vdash T \leq \exists x: S . T^{\prime}} & \frac{\Gamma, x: S \vdash T \leq T^{\prime} \quad x \notin F V\left(T^{\prime}\right)}{\Gamma \vdash \exists x: S . T \leq T^{\prime}}
\end{array}
\end{aligned}
$$

Figure 18: Subtyping Rules

\section{Runtime Typing Rules}

$$
\Sigma \vdash v: T \quad \Sigma \vdash_{H} O: T
$$
RT-T-LOC
$\frac{\Sigma(l)=T}{\Sigma+l: T}$
RT-T-CONST
$\Sigma \vdash v: \operatorname{ty}(c)$

RT-T-OB J

$\frac{\lfloor\Sigma(l)\rfloor=C \quad \text { fieldDefs }(H, l)=\circ \bar{f}:=\bar{v}_{\mathrm{I}}, \square \bar{g}:=\bar{v}_{\mathrm{M}} \quad \Sigma \vdash \bar{v}_{\mathrm{I}}: \bar{T}_{\mathrm{I}}}{\Sigma \vdash_{H}\{\text { proto: } l ; f: \widetilde{F}\}: \exists \bar{z}_{\mathrm{I}}: \bar{T}_{\mathrm{I}} \cdot\left\{\nu: C \mid \nu \cdot \bar{f}=\bar{z}_{\mathrm{I}} \wedge \operatorname{inv}(C, \nu)\right\}}$

Figure 19: Typing Runtime Configurations for IRSC 


\section{B. Proofs}

The main results in this section are:

- Program Consistency Lemma (Lemma 13, page 34)

- Forward Simulation Theorem (Theorem 2, page 38)

- Subject Reduction Theorem (Theorem 3, page 40)

- Progress Theorem (Theorem 4, page 47)

\section{B.1 SSA Translation}

Definition 2 (Environment Substitution).

$$
\left[\delta_{1} / \delta_{2}\right] \doteq\left[\bar{x}_{1} / \bar{x}_{2}\right] \quad \text { where }\left(\overline{\mathrm{x}}, \bar{x}_{1}, \bar{x}_{2}\right)=\delta_{1} \bowtie \delta_{2}
$$

Definition 3 (Valid Configuration).

$$
\text { validConf }(\mathrm{K} ; \mathrm{w}) \doteq \begin{cases}\text { true } & \text { if }(\mathrm{K} . \mathrm{X}=\cdot) \Rightarrow \exists \mathrm{B} \text { s.t. } \mathrm{w} \equiv \mathrm{B} \\ \text { false } & \text { otherwise }\end{cases}
$$

Assumption 1 (Stack Form). Let stack $\mathrm{X}=\mathrm{X}_{0} ; \mathrm{L}, \mathrm{E}$. Evaluation context $\mathrm{E}$ is of one of the following forms:

- $\mathrm{E}_{0} ;$ return e

- return $\mathrm{E}_{0}$

Lemma 1 (Global Environment Substitution). If $\mathrm{L} ; \mathrm{e} \stackrel{\mathrm{H}, \Delta}{\longrightarrow} e$, then $\mathrm{L} ; \mathrm{e} \stackrel{\mathrm{H}, \Delta^{\prime}}{\longrightarrow}\left[\Delta^{\prime}(\mathrm{e}) / \Delta(\mathrm{e})\right] e$

Lemma 2 (Evaluation Context). If

$$
\mathrm{L} ; \mathrm{w} \stackrel{\mathrm{H}, \Delta}{\longrightarrow} E[e]
$$

then there exist $\mathrm{E}$ and e s.t.:

- $\mathrm{w} \equiv \mathrm{E}[\mathrm{e}]$

- L; $\mathrm{E} \stackrel{\mathrm{H}, \Delta}{\longrightarrow} E$

- $\mathrm{L} ; \mathrm{e} \stackrel{\mathrm{H}, \Delta}{\longrightarrow} e$

Proof. By induction on the derivation of the input transformation.

Lemma 3 (Translation under Store). If $\cdot ; \mathrm{B} \stackrel{\cdot, \Delta}{\longrightarrow} e$, then $\mathrm{L} ; \mathrm{B} \stackrel{\mathrm{H}, \Delta}{\longrightarrow} \theta$, where $\theta=$ toSubst $(\Delta(\mathrm{B}), \mathrm{L}, \mathrm{H})$.

Proof. By induction on the structure of the input translation.

Lemma 4 (Canonical Forms).

(a) If L; $\stackrel{\mathrm{H}, \Delta}{\longrightarrow} c$, then $\mathrm{w} \equiv \mathrm{c}$

(b) If $\mathrm{L} ; \mathrm{w} \stackrel{\mathrm{H}, \Delta}{\longrightarrow} \operatorname{l} . m(\bar{v})$, then $\mathrm{w} \equiv \operatorname{l.m}(\overline{\mathrm{v}})$

(c) If L; $\stackrel{\mathrm{H}, \Delta}{\longrightarrow}$ letif $[\bar{\phi}](e) ? u_{1}: u_{2}$ in $e^{\prime}$, then $\mathrm{w} \equiv \operatorname{if}(\mathrm{e})\left\{\mathrm{s}_{1}\right\}$ else $\left\{\mathrm{s}_{2}\right\}$; return $\mathrm{e}^{\prime}$

(d) If $\widetilde{\mathrm{M}} \hookrightarrow \operatorname{def} m(\bar{x})=e_{0}$, then $\widetilde{\mathrm{M}} \equiv \mathrm{m}(\overline{\mathrm{x}})\{\mathrm{B}\}$

Lemma 5 (Translation Closed under Evaluation Context Composition). If

(a) $\mathrm{L} ; \mathrm{E}_{0} \stackrel{\mathrm{H}, \Delta}{\longrightarrow} E_{0}$

(b) $\mathrm{L}^{\prime} ;\left(\mathrm{L} ; \mathrm{E}_{1}\right) ; \mathrm{B} \stackrel{\mathrm{H}, \Delta}{\longrightarrow} e$

then $\mathrm{L}^{\prime} ;\left(\mathrm{L} ; \mathrm{E}_{0}\left[\mathrm{E}_{1}\right]\right) ; \mathrm{B} \stackrel{\mathrm{H}, \Delta}{\longrightarrow} E_{0}[e]$

Lemma 6 (Heap and Store Weakening). If

$$
\mathrm{L} ; \mathrm{X} ; \mathrm{E} \stackrel{\mathrm{H}, \Delta}{\longrightarrow} W
$$


then $\forall \mathrm{H}^{\prime}, \mathrm{L}^{\prime}$ s.t. $\mathrm{H}^{\prime} \supseteq \mathrm{H}$ and $\mathrm{L}^{\prime} \supseteq \mathrm{L}$, it holds that $\mathrm{L}^{\prime} ; \mathrm{X} ; \mathrm{E} \stackrel{\mathrm{H}^{\prime}, \Delta}{\longrightarrow} W$

Lemma 7 (Translation Closed under Stack Extension). If

(a) $\mathrm{L}_{0} ; \mathrm{X}_{0} ; \mathrm{E}_{0} \stackrel{\mathrm{H}, \Delta}{\longrightarrow} E_{0}$

(b) $\mathrm{L}_{1} ; \mathrm{X}_{1} ; \mathrm{B}_{1} \stackrel{\mathrm{H}, \Delta}{\longrightarrow} e_{1}$

then $\mathrm{L}_{1} ;\left(\mathrm{X}_{0} ; \mathrm{L}_{0}, \mathrm{E}_{0} ; \mathrm{X}_{1}\right) ; \mathrm{B}_{1} \stackrel{\mathrm{H}, \Delta}{\longrightarrow} E_{0}\left[e_{1}\right]$

Proof. We proceed by induction on the structure of derivation (b):

- [S-STACK-EMP]: Fact (b) has the form:

$$
\mathrm{L}_{1} ; \cdot ; \mathrm{B}_{1} \stackrel{\mathrm{H}, \Delta}{\longrightarrow} e_{1}
$$

By applying Rule S-STACK-CONS on 2.1 and (a):

$$
\mathrm{L}_{1} ;\left(\mathrm{X}_{0} ; \mathrm{L}_{0}, \mathrm{E}_{0}\right) ; \mathrm{B}_{1} \stackrel{\mathrm{H}, \Delta}{\longrightarrow} E_{0}\left[e_{1}\right]
$$

Which proves the wanted result.

- [S-STACK-CONS]: Fact (b) has the form:

$$
\mathrm{L}_{1} ;(\mathrm{X} ; \mathrm{L}, \mathrm{E}) ; \mathrm{B}_{1} \stackrel{\mathrm{H}, \Delta}{\longrightarrow} E\left[e_{1.1}\right]
$$

By inverting Rule S-STACK-CONS on 2.3:

$$
\begin{array}{r}
\mathrm{L}_{1} ; \cdot ; \mathrm{B}_{1} \stackrel{\mathrm{H, \Delta}}{\stackrel{\longrightarrow}{\longrightarrow} e_{1.1}} \\
\mathrm{~L} ; \mathrm{X} ; \mathrm{E} \stackrel{\mathrm{H}, \Delta}{\longrightarrow} E
\end{array}
$$

By induction hypothesis on (a) and 2.5 (the lemma can easily be extended to evaluation contexts):

$$
\mathrm{L} ;\left(\mathrm{X}_{0} ; \mathrm{L}_{0}, \mathrm{E}_{0} ; \mathrm{X}\right) ; \mathrm{E} \stackrel{\mathrm{H}, \Delta}{\longrightarrow} E_{0}[E]
$$

By applying Rule S-EC-STACK-CONS on 2.4 and 2.6:

$$
\mathrm{L}_{1} ;\left(\mathrm{X}_{0} ; \mathrm{L}_{0}, \mathrm{E}_{0} ; \mathrm{X} ; \mathrm{L}, \mathrm{E}\right) ; \mathrm{B}_{1} \stackrel{\mathrm{H}, \Delta}{\longrightarrow} E_{0}\left[E\left[e_{1.1}\right]\right]
$$

Which proves the wanted result.

Lemma 8 (Translation Closed under Evaluation Context Application). If

(a) $\mathrm{L} ; \mathrm{X} ; \mathrm{E} \stackrel{\mathrm{H}, \Delta}{\longrightarrow} W$

(b) $\mathrm{L} ; \mathrm{e} \stackrel{\mathrm{H}, \Delta}{\longrightarrow} e$

then $\mathrm{L} ; \mathrm{X} ; \mathrm{E}[\mathrm{e}] \stackrel{\mathrm{H}, \Delta}{\longrightarrow} W[e]$

Proof. By induction on the derivation of $(a)$.

Lemma 9 (Method Resolution). If

(a) $\mathrm{K} ; \mathrm{H} \hookrightarrow H$

(b) $\mathrm{H} ; \mathrm{I} \hookrightarrow l$

(c) toString $(\mathrm{m})=$ toString $(m)$

(d) resolveMethod $(H, l, m)=\widetilde{M}$

then: 
(e) resolve_method $(\mathrm{H}, 1, \mathrm{~m})=\widetilde{\mathrm{M}}$

(f) $\widetilde{\mathrm{M}} \hookrightarrow \widetilde{M}$

Lemma 10 (Value Monotonicity). If

(a) validConf $(\mathrm{K} ; \mathrm{w})$

(b) $\mathrm{K} ; \mathrm{w} \stackrel{\Delta}{\hookrightarrow} K ; v$

then there exist $\mathrm{L}^{\prime}$ and $\mathrm{w}^{\prime}$ s.t.:

(c) $\mathrm{K} ; \mathrm{w} \longrightarrow{ }^{*} \mathrm{~K}^{\prime} ; \mathrm{w}^{\prime}$

(d) $\mathrm{K}^{\prime} ; \mathrm{w}^{\prime} \stackrel{\Delta}{\hookrightarrow} K ; v$

(e) $\mathrm{w}^{\prime} \equiv \begin{cases}\text { return } \mathrm{v} & \text { if } \mathrm{w} \equiv \mathrm{B} \\ \mathrm{v} & \text { otherwise }\end{cases}$

(f) If $\mathrm{K} . \mathrm{X}=\cdot$ then $\mathrm{K}^{\prime} . \mathrm{L}=\mathrm{K} . \mathrm{L}$

where $\mathrm{K}^{\prime} \equiv \mathrm{K} . \mathrm{S} ; \mathrm{L}^{\prime} ; \cdot ; \mathrm{K} . \mathrm{H}$

Proof. By induction on the structure of the derivation (b).

Lemma 11 (Top-Level Reduction). If

$\mathrm{S} ; \mathrm{L} ; \mathrm{X} ; \mathrm{H} ; \mathrm{w} \longrightarrow \mathrm{S} ; \mathrm{L}^{\prime} ; \mathrm{X}^{\prime} ; \mathrm{H}^{\prime} ; \mathrm{w}^{\prime}$

then for a stack $\mathrm{X}_{0}$ it holds that:

$\mathrm{S} ; \mathrm{L} ;\left(\mathrm{X}_{0} ; \mathrm{X}\right) ; \mathrm{H} ; \mathrm{w} \longrightarrow \mathrm{S} ; \mathrm{L}^{\prime} ;\left(\mathrm{X}_{0} ; \mathrm{X}^{\prime}\right) ; \mathrm{H}^{\prime} ; \mathrm{w}^{\prime}$

Proof. By induction on the structure of the input reduction.

Lemma 12 (Empty Stack Consistency). If

(a) $\mathrm{K} ; \mathrm{w} \stackrel{\Delta}{\hookrightarrow} K ; e$

(b) $\mathrm{K} . \mathrm{X}=$.

(c) $K ; e \longrightarrow K^{\prime} ; e^{\prime}$

then there exist $\mathrm{K}^{\prime}$ and $\mathrm{w}^{\prime}$ s.t.:

(d) $\mathrm{K} ; \mathrm{w} \longrightarrow{ }^{*} \mathrm{~K}^{\prime} ; \mathrm{w}^{\prime}$,

(e) $\mathrm{K}^{\prime} ; \mathrm{w}^{\prime} \stackrel{\Delta}{\hookrightarrow} K^{\prime} ; e^{\prime}$

(f) $\triangleright$ If $\mathrm{w} \equiv \mathrm{E}[\mathrm{I} \cdot \mathrm{m}(\overline{\mathrm{v}})]$ then:

$-\mathrm{K}^{\prime} . \mathrm{X}=\mathrm{K} . \mathrm{L}, \mathrm{E}$

$-\mathrm{K}^{\prime} . \mathrm{H}=\mathrm{K} . \mathrm{H}$

$-\exists \mathrm{B}^{\prime}$ s.t. $\mathrm{w}^{\prime} \equiv \mathrm{B}^{\prime}$

$-K^{\prime}=K$

$\triangleright$ Otherwise:

$-\mathrm{K}^{\prime} . \mathrm{X}=$.

$-K^{\prime} . H \supseteq K . H$

$-K^{\prime} . L \supseteq K . L$

- If $\exists \mathrm{e}$ s.t. $\mathrm{w} \equiv \mathrm{e}$ then $\exists \mathrm{e}^{\prime}$ s.t. $\mathrm{w}^{\prime} \equiv \mathrm{e}^{\prime}$

- If $\exists \mathrm{B}$ s.t. $\mathrm{w} \equiv \mathrm{B}$ then $\exists \mathrm{B}^{\prime}$ s.t. $\mathrm{w}^{\prime} \equiv \mathrm{B}^{\prime}$

Proof. Fact (a) has the form:

$$
\mathrm{K} ; \mathrm{w} \stackrel{\Delta}{\hookrightarrow} S ; H ; e
$$

Because of fact (b):

$$
\mathrm{K} \equiv \mathrm{S} ; \mathrm{L} ; \cdot ; \mathrm{H}
$$


By inverting Rule S-EXP-RTCONF on 6.1:

$$
\begin{array}{r}
\mathrm{S} \stackrel{\Delta}{\hookrightarrow} S \\
\mathrm{~K} ; \mathrm{H} \stackrel{\hookrightarrow}{\hookrightarrow} H \\
\mathrm{~L} ; \cdot ; \mathrm{w} \stackrel{\mathrm{H}, \Delta}{\hookrightarrow} e
\end{array}
$$

By inverting S-STACK-EMP on 6.10:

$$
\mathrm{L} ; \mathrm{w} \stackrel{\mathrm{H}, \Delta}{\longrightarrow} e
$$

Suppose w is a value. By Rules S-CONST and S-LOC, $e$ is also a value: a contradiction because of (c). Hence:

$$
\text { w not a value }
$$

We proceed by induction on the structure of reduction (c):

$\cdot[\mathrm{RC}-\mathrm{ECTX}]$

$$
K ; E_{0}\left[e_{0}\right] \longrightarrow K^{\prime} ; E_{0}\left[e_{0}^{\prime}\right]
$$

By inverting RC-ECTX on 6.8:

$$
K ; e_{0} \longrightarrow K^{\prime} ; e_{0}^{\prime}
$$

Fact 6.6 is of the form:

$$
\mathrm{L} ; \mathrm{w} \stackrel{\mathrm{H}, \Delta}{\longrightarrow} E_{0}\left[e_{0}\right]
$$

By Lemma 2 on 6.10:

$$
\begin{gathered}
\mathrm{w} \equiv \mathrm{E}_{0}\left[\mathrm{e}_{0}\right] \\
\mathrm{L} ; \mathrm{E}_{0} \stackrel{\stackrel{\mathrm{H}, \Delta}{\longrightarrow} E_{0}}{\mathrm{~L} ; \mathrm{e}_{0}} \stackrel{\stackrel{\mathrm{H}, \Delta}{\longrightarrow} e_{0}}{ }
\end{gathered}
$$

By applying Rule S-STACK-EMP on 6.13:

$$
\mathrm{L} ; \cdot ; \mathrm{e}_{0} \stackrel{\mathrm{H}, \Delta}{\longrightarrow} e_{0}
$$

By applying Rule S-EXP-RTCONF on 6.3, 6.4 and 6.14:

$$
\mathrm{K} ; \mathrm{e}_{0} \stackrel{\Delta}{\hookrightarrow} K ; e_{0}
$$

By induction hypothesis using 6.15, (b) and 6.9:

$$
\begin{array}{r}
\mathrm{S} ; \mathrm{L} ; \cdot ; \mathrm{H} ; \mathrm{e}_{0} \longrightarrow \mathrm{S} ; \mathrm{L}^{\prime} ; \mathrm{X}^{\prime} ; \mathrm{H}^{\prime} ; \mathrm{w}_{0}^{\prime} \\
\mathrm{S} ; \mathrm{L}^{\prime} ; \mathrm{X}^{\prime} ; \mathrm{H}^{\prime} ; \mathrm{w}_{0}^{\prime} \stackrel{\Delta}{\hookrightarrow} K^{\prime} ; e_{0}^{\prime}
\end{array}
$$

We examine cases on the form of $e_{0}$ :

- Case $\mathrm{e}_{0} \equiv \mathrm{E}_{1}[\mathrm{l} . \mathrm{m}(\overline{\mathrm{v}})]$ :

$$
\begin{aligned}
\mathrm{X}^{\prime} & =\mathrm{L} ; \mathrm{E}_{1} \\
\mathrm{H}^{\prime} & =\mathrm{H} \\
\mathrm{w}_{0}^{\prime} & =\mathrm{B}^{\prime} \\
K^{\prime} & =K
\end{aligned}
$$


For some method body B'. So 6.17 becomes:

$$
\mathrm{S} ; \mathrm{L}^{\prime} ;\left(\mathrm{L} ; \mathrm{E}_{1}\right) ; \mathrm{H} ; \mathrm{B}^{\prime} \stackrel{\Delta}{\hookrightarrow} K ; e_{0}^{\prime}
$$

By inverting rule R-CALL on 6.16:

$$
\begin{array}{r}
\operatorname{resolve\_ method}(\mathrm{H}, \mathrm{l}, \mathrm{m})=\mathrm{m}(\overline{\mathrm{x}})\left\{\mathrm{B}^{\prime}\right\} \\
\mathrm{L}^{\prime}=\overline{\mathrm{x}} \mapsto \overline{\mathrm{v}} ; \text { this } \mapsto \mathrm{I} \\
\mathrm{X}_{0}^{\prime}=\mathrm{L}, \mathrm{E}_{1}
\end{array}
$$

By applying rule $\mathrm{R}-\mathrm{CALL}$ using $6.23,6.24$ and $\mathrm{X}^{\prime}=\mathrm{L}, \mathrm{E}_{0}\left[\mathrm{E}_{1}\right]$ on $\mathrm{K} ; \mathrm{w} \equiv \mathrm{S} ; \mathrm{L} ; \cdot ; \mathrm{H} ;\left(\mathrm{E}_{0}\left[\mathrm{E}_{1}\right]\right)[1 . \mathrm{m}(\overline{\mathrm{V}})]$ :

$$
\mathrm{S} ; \mathrm{L} ; \cdot ; \mathrm{H} ;\left(\mathrm{E}_{0}\left[\mathrm{E}_{1}\right]\right)[1 . \mathrm{m}(\overline{\mathrm{V}})] \longrightarrow \mathrm{S} ; \mathrm{L}^{\prime} ;\left(\mathrm{L} ; \mathrm{E}_{0}\left[\mathrm{E}_{1}\right]\right) ; \mathrm{H} ; \mathrm{B}^{\prime}
$$

Which proves (d). By inverting Rule S-EXP-RTCONF on 6.22:

$$
\begin{array}{r}
\mathrm{K}^{\prime} ; \mathrm{H} \hookrightarrow H \\
\mathrm{~L}^{\prime} ;\left(\mathrm{L} ; \mathrm{E}_{1}\right) ; \mathrm{B}^{\prime} \stackrel{\mathrm{H}, \Delta}{\longrightarrow} e_{0}^{\prime}
\end{array}
$$

From Lemma 5 on 6.12 and 6.28:

$$
\mathrm{L}^{\prime} ;\left(\mathrm{L} ; \mathrm{E}_{0}\left[\mathrm{E}_{1}\right]\right) ; \mathrm{B}^{\prime} \stackrel{\mathrm{H}, \Delta}{\longrightarrow} E_{0}\left[e_{0}^{\prime}\right]
$$

By applying rule S-EXP-RTCONF using 6.3, 6.27 and 6.29:

$$
\mathrm{S} ; \mathrm{L}^{\prime} ;\left(\mathrm{L} ; \mathrm{E}_{0}\left[\mathrm{E}_{1}\right]\right) ; \mathrm{H} ; \mathrm{B}^{\prime} \stackrel{\Delta}{\hookrightarrow} K ; E_{0}\left[e_{0}^{\prime}\right]
$$

Which proves (e). By 6.11 and the current case:

$$
\mathrm{w} \equiv\left(\mathrm{E}_{0}\left[\mathrm{E}_{1}\right]\right)[1 . \mathrm{m}(\overline{\mathrm{v}})]
$$

By 6.26 and 6.30:

$$
\begin{array}{r}
\mathrm{K}^{\prime} . \mathrm{X}=\mathrm{L} ; \mathrm{E}_{0}\left[\mathrm{E}_{1}\right] \\
\mathrm{w}^{\prime}=\mathrm{B}^{\prime} \\
K^{\prime}=K
\end{array}
$$

By $6.32,6.19,6.33$ and 6.34 we prove (f).

- All remaining cases:

$$
\begin{gathered}
\mathrm{X}^{\prime} \equiv \\
\mathrm{H}^{\prime} \supseteq \mathrm{H} \\
\mathrm{L}^{\prime} \supseteq \mathrm{L} \\
\mathrm{w}_{0}^{\prime} \equiv \mathrm{e}_{0}^{\prime}
\end{gathered}
$$

So 6.16 and 6.17 become:

$$
\begin{array}{r}
\mathrm{S} ; \mathrm{L} ; \cdot ; \mathrm{H} ; \mathrm{e}_{0} \longrightarrow \mathrm{S} ; \mathrm{L}^{\prime} ; \cdot \cdot ; \mathrm{H}^{\prime} ; \mathrm{e}_{0}^{\prime} \\
\mathrm{S} ; \mathrm{L}^{\prime} ; \cdot ; \mathrm{H}^{\prime} ; \mathrm{e}_{0}^{\prime} \stackrel{\Delta}{\hookrightarrow} K^{\prime} ; e_{0}^{\prime}
\end{array}
$$

By applying Rule R-EvaLCTX using 6.39:

$$
\mathrm{S} ; \mathrm{L} ; \cdot ; \mathrm{H} ; \mathrm{E}_{0}\left[\mathrm{e}_{0}\right] \longrightarrow \mathrm{S} ; \mathrm{L}^{\prime} ; \cdot ; \mathrm{H}^{\prime} ; \mathrm{E}_{0}\left[\mathrm{e}_{0}^{\prime}\right]
$$


Which proves (d) and (f). By inverting Rules S-EXP-RTCONF and S-STACK-EMP on 6.40:

$$
L^{\prime} ; e_{0} \stackrel{H^{\prime}, \Delta}{\longrightarrow} e_{0}
$$

From Lemma 6 using 6.12, 6.36 and 6.37:

$$
\mathrm{L}^{\prime} ; \mathrm{E}_{0} \stackrel{\mathrm{H}^{\prime}, \Delta}{\longrightarrow} E_{0}
$$

From Lemma 8 on 6.42 and 6.43:

$$
\mathrm{L}^{\prime} ; \mathrm{E}_{0}\left[\mathrm{e}_{0}\right] \stackrel{\mathrm{H}^{\prime}, \Delta}{\longrightarrow} E_{0}\left[e_{0}\right]
$$

By inverting rule S-EXP-RTCONF on 6.40:

$$
\mathrm{K}^{\prime} ; \mathrm{H}^{\prime} \hookrightarrow H^{\prime}
$$

By Rule S-EXP-RTCONF using 6.3, 6.44 and 6.45:

$$
\mathrm{S} ; \mathrm{L}^{\prime} ; \cdot ; \mathrm{H}^{\prime} ; \mathrm{E}_{0}\left[\mathrm{e}_{0}^{\prime}\right] \stackrel{\Delta}{\hookrightarrow} S ; H^{\prime} ; E_{0}\left[e_{0}^{\prime}\right]
$$

Which proves (e).

- [R-CALL]:

$$
K ; l . m(\bar{v}) \longrightarrow K ;[\bar{v} / \bar{x}, l / \text { this }] e_{0}
$$

Where by inverting R-CALL on 6.47:

$$
\text { resolveMethod }(H, l, m)=\left(\operatorname{def} m(\bar{x})=e_{0}\right)
$$

Fact 6.5 is of the form:

$$
\mathrm{L} ; \cdot ; \mathrm{w} \stackrel{\mathrm{H}, \Delta}{\longrightarrow} l . m(\bar{v})
$$

By Lemma 4(b) on 6.49:

$$
\mathrm{w} \equiv \operatorname{l} . \mathrm{m}(\overline{\mathrm{v}})
$$

So 6.49 becomes:

$$
\mathrm{L} ; \cdot ; \operatorname{l} . \mathrm{m}(\overline{\mathrm{v}}) \stackrel{\mathrm{H}, \Delta}{\longrightarrow} \operatorname{l.m}(\bar{v})
$$

By inverting Rule S-STACK-EMP on 6.51:

$$
\mathrm{L} ; 1 . \mathrm{m}(\overline{\mathrm{v}}) \stackrel{\mathrm{H}, \Delta}{\longrightarrow} \operatorname{l} . m(\bar{v})
$$

By inverting Rule SR-CALL on 6.52:

$$
\begin{array}{r}
\mathrm{L} ; \mathrm{I} \stackrel{\stackrel{\mathrm{H}, \Delta}{\longrightarrow} l}{\mathrm{~L} ; \overline{\mathrm{V}} \stackrel{\mathrm{H}, \Delta}{\longrightarrow} \bar{v}} \\
\text { toString }(\mathrm{m})=\text { toString }(m)
\end{array}
$$

By inverting SR-VAL on 6.53 and 6.54:

$$
\begin{array}{r}
\mathrm{H} ; \mathrm{I} \hookrightarrow l \\
\mathrm{H} ; \overline{\mathrm{V}} \hookrightarrow \bar{v}
\end{array}
$$


By Lemma 9 on 6.4, 6.56, 6.55 and 6.48:

$$
\begin{array}{r}
\operatorname{resolve\_ method}(\mathrm{H}, \mathrm{I}, \mathrm{m})=\widetilde{\mathrm{M}} \\
\widetilde{\mathrm{M}} \stackrel{\Delta}{\hookrightarrow} \operatorname{def} m(\bar{x})=e_{0}
\end{array}
$$

By Lemma 4(d) on 6.59:

$$
\widetilde{\mathrm{M}} \equiv \mathrm{m}(\overline{\mathrm{X}})\{\mathrm{B}\}
$$

By applying Rule R-CALL using 6.58, 6.63, 6.64 and E $\equiv[$ ]:

$$
\mathrm{S} ; \mathrm{L} ; \mathrm{X} ; \mathrm{H} ; \mathrm{I} . \mathrm{m}(\overline{\mathrm{V}}) \longrightarrow \mathrm{S} ; \mathrm{L}^{\prime} ; \mathrm{X}^{\prime} ; \mathrm{H} ; \mathrm{B}
$$

Which proves (d). By inverting rule SR-METH on 6.59:

$$
\cdot ; \mathrm{B} \stackrel{\cdot, \Delta}{\longrightarrow} e
$$

Let a store $\mathrm{L}^{\prime}$ and a stack $\mathrm{X}^{\prime}$ s.t.:

$$
\begin{array}{r}
\mathrm{L}^{\prime} \equiv \overline{\mathrm{x}} \mapsto \overline{\mathrm{v}} ; \text { this } \mapsto \mathrm{I} \\
\mathrm{x}^{\prime} \equiv \mathrm{L},[]
\end{array}
$$

By applying Lemma 3 on 6.62

$$
\mathrm{L}^{\prime} ; \mathrm{B} \stackrel{\mathrm{H}, \Delta}{\longrightarrow} \theta e_{0}
$$

Where:

$$
\begin{aligned}
\theta & \doteq \operatorname{toSubst}\left(\Delta(\mathrm{B}), \mathrm{L}^{\prime}, \mathrm{H}\right) \\
& =\left\{[v / x] \mid \mathrm{x} \mapsto x \in \Delta(\mathrm{B}), \mathrm{x} \mapsto \mathrm{v} \in \mathrm{L}^{\prime}, \mathrm{H} ; \mathrm{v} \hookrightarrow v\right\} \\
& =[\bar{v} / \bar{x}, l / \text { this }]
\end{aligned}
$$

We pick:

$$
\mathrm{w}^{\prime} \equiv \mathrm{B}
$$

By applying Rule S-STACK-EMP using 6.65:

$$
\mathrm{L}^{\prime} ; \cdot ; \mathrm{B} \stackrel{\mathrm{H}, \Delta}{\longrightarrow} \theta e_{0}
$$

It holds that:

$$
L ; ; ;[\stackrel{H, \Delta}{\longrightarrow}[]
$$

By Rule S-STACK-CONS on 6.68 and 6.69:

$$
\mathrm{L}^{\prime} ;(\mathrm{L},[]) ; \mathrm{B} \stackrel{\mathrm{H}, \Delta}{\longrightarrow} \theta e_{0}
$$

By Rule S-EXP-RTCONF using 6.3, 6.4 and 6.70:

$$
\mathrm{S} ; \mathrm{L}^{\prime} ; \mathrm{X}^{\prime} ; \mathrm{H} ; \mathrm{B} \stackrel{\Delta}{\hookrightarrow} S ; H ; \theta e_{0}
$$

Which proves (e). From 6.64, 6.61, 6.67 and 6.56 we prove (f). 
- [R-LETIF]:

$$
\begin{array}{r}
K \text {; letif }\left[\bar{x}, \bar{x}_{1}, \bar{x}_{2}\right](c) ? u_{1}: u_{2} \text { in } e_{0} \longrightarrow K ; u_{i}\left\langle\left[\bar{x}_{i} / \bar{x}\right] e_{0}\right\rangle \\
c=\text { true } \Rightarrow i=1 \\
c=\text { false } \Rightarrow i=2
\end{array}
$$

Let:

$$
c=\text { true }
$$

The case for false is symmetrical. Facts 6.72 and 6.6 become:

$$
\begin{array}{r}
K ; \text { letif }\left[\bar{x}, \bar{x}_{1}, \bar{x}_{2}\right]\left(\text { true) } ? u_{1}: u_{2} \text { in } e_{0} \longrightarrow K ; u_{1}\left\langle\left[\bar{x}_{1} / \bar{x}\right] e_{0}\right\rangle\right. \\
\text { L; } \text { w } \stackrel{\mathrm{H}, \Delta}{\longrightarrow} \text { letif }\left[\bar{x}, \bar{x}_{1}, \bar{x}_{2}\right] \text { (true) } ? u_{1}: u_{2} \text { in } e_{0}
\end{array}
$$

By Lemma 4(c) on 6.77:

$$
\mathrm{w} \equiv \operatorname{if}\left(\mathrm{e}_{c}\right)\left\{\mathbf{s}_{1}\right\} \text { else }\left\{\mathbf{s}_{2}\right\} ; \text { return } \mathbf{e}_{0}
$$

So 6.77 becomes:

$$
\text { L; if }\left(\mathbf{e}_{c}\right)\left\{\mathbf{s}_{1}\right\} \text { else }\left\{\mathbf{s}_{2}\right\} \text {; return } \mathrm{e}_{0} \stackrel{\mathrm{H}, \Delta}{\longrightarrow} \text { letif }\left[\bar{x}, \bar{x}_{1}, \bar{x}_{2}\right](\text { true }) ? u_{1}: u_{2} \text { in } e_{0}
$$

By inverting Rule SR-BODY on 6.79:

$$
\begin{array}{r}
\text { L; if }\left(\mathrm{e}_{c}\right)\left\{\mathbf{s}_{1}\right\} \text { else }\left\{\mathbf{s}_{2}\right\} \stackrel{\mathrm{H}, \Delta}{\longrightarrow} \text { letif }\left[\bar{x}, \bar{x}_{1}, \bar{x}_{2}\right](\text { true }) ? u_{1}: u_{2} \text { in }\langle\rangle \\
\Delta^{\prime}=\Delta\left[\mathrm{e}_{0} \mapsto \Delta\left\lfloor\text { if }\left(\mathrm{e}_{c}\right)\left\{\mathbf{s}_{1}\right\} \text { else }\left\{\mathbf{s}_{2}\right\}\right\rfloor\right] \\
\mathrm{L} ; \mathrm{e}_{0} \stackrel{\mathrm{H}, \Delta^{\prime}}{\longrightarrow} e_{0}
\end{array}
$$

By inverting Rule SR-ITE on 6.80:

$$
\begin{array}{r}
\mathrm{L} ; \mathrm{e}_{c} \stackrel{\mathrm{H}, \Delta}{\stackrel{\mathrm{H}}{\longrightarrow} \text { true }} \\
\mathrm{L} ; \mathrm{s}_{1} \stackrel{\mathrm{H}, \Delta}{\longrightarrow} u_{1} \\
\mathrm{~L} ; \mathrm{s}_{2} \stackrel{\mathrm{H}, \Delta}{\longrightarrow} u_{2} \\
\left(\overline{\mathrm{x}}, \bar{x}_{1}, \bar{x}_{2}\right)=\Delta\left\lfloor\mathrm{s}_{1}\right\rfloor \Delta\left\lfloor\mathrm{s}_{2}\right\rfloor \\
\bar{x}=\Delta\left\lfloor\text { if }\left(\mathrm{e}_{c}\right)\left\{\mathrm{s}_{1}\right\} \text { else }\left\{\mathbf{s}_{2}\right\}\right\rfloor(\overline{\mathrm{x}})
\end{array}
$$

By Lemma 4 on 6.83 we get:

$$
\mathrm{e}_{c} \equiv \text { true }
$$

By Rules R-EvalCTX and R-ITE we get:

$$
\mathrm{K} ; \text { if(true) }\left\{\mathbf{s}_{1}\right\} \text { else }\left\{\mathbf{s}_{2}\right\} \text {; return } \mathrm{e}_{0} \longrightarrow \mathrm{K} ; \mathbf{s}_{1} \text {; return } \mathrm{e}_{0}
$$

Which proves (d). Let:

$$
\Delta^{\prime \prime} \equiv \Delta^{\prime}\left[\mathrm{e}_{0} \mapsto \Delta\left\lfloor\mathbf{s}_{1}\right\rfloor\right]
$$

By Lemma 1 on 6.82 using 6.90:

$$
\mathrm{L} ; \mathrm{e}_{0} \stackrel{\mathrm{H}, \Delta^{\prime \prime}}{\longrightarrow}\left[\Delta^{\prime \prime}\left(\mathrm{e}_{0}\right) / \Delta^{\prime}\left(\mathrm{e}_{0}\right)\right] e_{0}
$$


From 6.81 and 6.90 it holds that:

$$
\begin{aligned}
\Delta^{\prime}\left(\mathrm{e}_{0}\right) & =\Delta\left\lfloor\text { if }(\text { true })\left\{\mathbf{s}_{1}\right\} \text { else }\left\{\mathbf{s}_{2}\right\}\right\rfloor \\
\Delta^{\prime \prime}\left(\mathrm{e}_{0}\right) & =\Delta\left\lfloor\mathbf{s}_{1}\right\rfloor
\end{aligned}
$$

So:

$$
\Delta^{\prime}\left(\mathrm{e}_{0}\right) \bowtie \Delta^{\prime \prime}\left(\mathrm{e}_{0}\right)=\left(\overline{\mathrm{x}}, \bar{x}_{1}, \bar{x}\right)
$$

By Definition 2:

$$
\left[\Delta^{\prime \prime}\left(\mathrm{e}_{0}\right) / \Delta^{\prime}\left(\mathrm{e}_{0}\right)\right]=\left[\bar{x}_{1} / \bar{x}\right]
$$

So 6.91 becomes:

$$
\mathrm{L} ; \mathrm{e}_{0} \stackrel{\mathrm{H}, \Delta^{\prime \prime}}{\longrightarrow}\left[\bar{x}_{1} / \bar{x}\right] e_{0}
$$

By applying Rule SR-BODY on 6.84, 6.93 and 6.96, using 6.95:

$$
\mathrm{L} ; \mathbf{s}_{1} ; \text { return } \mathrm{e}_{0} \stackrel{\mathrm{H}, \Delta}{\longrightarrow} u_{1}\left\langle\left[\bar{x}_{1} / \bar{x}\right] e_{0}\right\rangle
$$

Which, using S-EXP-RTCONF and S-STACK-EMP, prove (e) and (f).

- [R-CAST], [R-NEw], [R-LETIN], [R-DotASGN], [R-FIELD]: Cases handled in similar fashion as before.

Corollary 1 (Empty Stack Valid Configuration). If
(a) $\mathrm{K} ; \mathrm{w} \stackrel{\Delta}{\hookrightarrow} K ; e$
(b) $\mathrm{K} . \mathrm{X}=$.
(c) $K ; e \longrightarrow K^{\prime} ; e^{\prime}$

then $\mathrm{K} ; \mathrm{w} \longrightarrow{ }^{*} \mathrm{~K}^{\prime} ; \mathrm{w}^{\prime}$ with validConf $\left(\mathrm{K}^{\prime} ; \mathrm{w}^{\prime}\right)$.

Proof. Examine all cases of result (f) of Lemma 12.

Lemma 13 (Consistency). If

(a) $\mathrm{K} ; \mathrm{w} \stackrel{\Delta}{\hookrightarrow} K ; e$

(b) $K ; e \longrightarrow K^{\prime} ; e^{\prime}$

(c) validConf $(\mathrm{K} ; \mathrm{w})$

then there exist $\mathrm{K}^{\prime}$ and $\mathrm{w}^{\prime}$ s.t.:

(d) $\mathrm{K} ; \mathrm{w} \longrightarrow{ }^{*} \mathrm{~K}^{\prime} ; \mathrm{w}^{\prime}$,

(e) $\mathrm{K}^{\prime} ; \mathrm{w}^{\prime} \stackrel{\Delta}{\hookrightarrow} K^{\prime} ; e^{\prime}$

(f) validConf $\left(\mathrm{K}^{\prime} ; \mathrm{w}^{\prime}\right)$

Proof. Let:

$$
\mathrm{K} \equiv \mathrm{S} ; \mathrm{L} ; \mathrm{X} ; \mathrm{H}
$$

By inverting Rule S-EXP-RTCONF on (a):

$$
\begin{array}{r}
\mathrm{S} \stackrel{\Delta}{\hookrightarrow} S \\
\mathrm{~K} ; \mathrm{H} \stackrel{\hookrightarrow}{\hookrightarrow} H \\
\mathrm{~L} ; \mathrm{X} ; \mathrm{w} \stackrel{\mathrm{H}, \Delta}{\longrightarrow} e
\end{array}
$$

We proceed by induction on the derivation 6.4: 
$\cdot[$ S-STACK-EMP]:

$$
\mathrm{L} ; \cdot ; \mathrm{w} \stackrel{\mathrm{H}, \Delta}{\longrightarrow} e
$$

By Lemma 12 using (a) and (b) there exist $\mathrm{w}^{\prime}$ and $\mathrm{K}^{\prime}$ s.t.:

$$
\begin{aligned}
& \mathrm{K} ; \mathrm{w} \longrightarrow^{*} \mathrm{~K}^{\prime} ; \mathrm{w}^{\prime} \\
& \mathrm{K}^{\prime} ; \mathrm{w}^{\prime} \stackrel{\Delta}{\hookrightarrow} K^{\prime} ; e^{\prime}
\end{aligned}
$$

From Corollary 1 using (a), (b) and (c) we get:

$$
\text { validConf }\left(\mathrm{K}^{\prime} ; \mathrm{w}^{\prime}\right)
$$

We prove (d), (e) and (f) by 6.6, 6.7 and 6.8, respectively.

- [S-STACK-CONS]:

$$
\mathrm{L} ;\left(\mathrm{X}_{0} ; \mathrm{L}_{0}, \mathrm{E}_{0}\right) ; \mathrm{w} \stackrel{\mathrm{H}, \Delta}{\longrightarrow} E_{0}\left[e_{0}\right]
$$

Where:

$$
\mathrm{X} \equiv \mathrm{X}_{0} ; \mathrm{L}_{0}, \mathrm{E}_{0}
$$

$\mathrm{By}(\mathrm{c})$ and the definition of a valid configuration, there exists a $\mathrm{B}_{0}$ s.t.:

$$
\mathrm{w} \equiv \mathrm{B}_{0}
$$

By inverting Rule S-STACK-CONS on 6.9 using 6.11:

$$
\begin{aligned}
\mathrm{L} ; \cdot ; \mathrm{B}_{0} & \stackrel{\mathrm{H}, \Delta}{\longleftrightarrow} e_{0} \\
\mathrm{~L}_{0} ; \mathrm{X}_{0} ; \mathrm{E}_{0} & \stackrel{\mathrm{H}, \Delta}{\longrightarrow} E_{0}
\end{aligned}
$$

By applying rule S-EXP-RTCONF on 6.2, 6.3 and 6.12:

$$
\mathrm{S} ; \mathrm{L} ; \cdot ; \mathrm{H} ; \mathrm{B}_{0} \stackrel{\Delta}{\hookrightarrow} S ; H ; e_{0}
$$

We examine cases on the configuration of $K ; e_{0}$ :

- Case $K ; e_{0}$ is a terminal configuration, so there exists $v$ s.t.:

$$
e_{0} \equiv v
$$

Fact 6.14 becomes:

$$
\mathrm{S} ; \mathrm{L} ; \cdot ; \mathrm{H} ; \mathrm{B}_{0} \stackrel{\Delta}{\hookrightarrow} S ; H ; v
$$

By Lemma 10 on 6.16:

$$
\begin{array}{r}
\mathrm{S} ; \mathrm{L} ; \cdot ; \mathrm{H} ; \mathrm{B}_{0} \longrightarrow{ }^{*} \mathrm{~S} ; \mathrm{L} ; \cdot ; \mathrm{H} ; \text { return } \mathrm{v} \\
\mathrm{S} ; \mathrm{L} ; \cdot ; \mathrm{H} ; \text { return } \mathrm{v} \stackrel{\Delta}{\hookrightarrow} K ; v
\end{array}
$$

By Lemma 11 on 6.17:

$$
\mathrm{S} ; \mathrm{L} ; \mathrm{X} ; \mathrm{H} ; \mathrm{B}_{0} \longrightarrow^{*} \mathrm{~S} ; \mathrm{L} ; \mathrm{X} ; \mathrm{H} ; \text { return v }
$$

By inverting Rule S-EXP-RTCONF on 6.18:

$$
\mathrm{L} ; \cdot ; \text { return } \mathrm{v} \stackrel{\mathrm{H}, \Delta}{\longrightarrow} v
$$


By applying Rule S-STACK-CONS on 6.20 and 6.13:

$$
\mathrm{L} ;\left(\mathrm{X}_{0} ; \mathrm{L}_{0}, \mathrm{E}_{0}\right) ; \text { return v } \stackrel{\mathrm{H}, \Delta}{\longrightarrow} E_{0}[v]
$$

By applying Rule S-EXP-RTCONF on 6.2, 6.3 and 6.21:

$$
\mathrm{S} ; \mathrm{L} ;\left(\mathrm{X}_{0} ; \mathrm{L}_{0}, \mathrm{E}_{0}\right) ; \mathrm{H} ; \text { return v } \stackrel{\Delta}{\hookrightarrow} S ; H ; E_{0}[v]
$$

By applying Rule R-RET on on THe left-hand side of 6.22:

$$
\mathrm{S} ; \mathrm{L} ;\left(\mathrm{X}_{0} ; \mathrm{L}_{0}, \mathrm{E}_{0}\right) ; \mathrm{H} ; \text { return } \mathrm{v} \longrightarrow \mathrm{S} ; \mathrm{L}_{0} ; \mathrm{X}_{0} ; \mathrm{H} ; \mathrm{E}_{0}[\mathrm{v}]
$$

By inverting S-STACK-EMP and SR-BODY on 6.20:

$$
\mathrm{L} ; \mathrm{v} \stackrel{\mathrm{H}, \Delta}{\longrightarrow} v
$$

By inverting Rule SR-VAL on 6.24:

$$
\mathrm{H} ; \mathrm{v} \hookrightarrow v
$$

By applying Rule SR-VAL on 6.25 using $\mathrm{L}_{0}$ :

$$
\mathrm{L}_{0} ; \mathrm{v} \stackrel{\mathrm{H}, \Delta}{\longrightarrow} v
$$

By applying Lemma 8 on 6.13 and 6.26:

$$
\mathrm{L}_{0} ; \mathrm{X}_{0} ; \mathrm{E}_{0}[\mathrm{v}] \stackrel{\mathrm{H}, \Delta}{\longrightarrow} E_{0}[v]
$$

By applying Rule S-EXP-RTCONF on 6.2, 6.3 and 6.27:

$$
\mathrm{S} ; \mathrm{L}_{0} ; \mathrm{X}_{0} ; \mathrm{H} ; \mathrm{E}_{0}[\mathrm{v}] \stackrel{\Delta}{\hookrightarrow} S ; H ; E_{0}[v]
$$

Because of 6.11:

$$
\operatorname{validConf}\left(\mathrm{S} ; \mathrm{L}_{0} ; \mathrm{X}_{0} ; \mathrm{H} ; \mathrm{E}_{0}[\mathrm{v}]\right)
$$

By induction hypothesis using 6.28, (b) and 6.29:

$$
\begin{array}{r}
\mathrm{S} ; \mathrm{L}_{0} ; \mathrm{X}_{0} ; \mathrm{H} ; \mathrm{E}_{0}[\mathrm{v}] \longrightarrow \mathrm{K}^{\prime} ; \mathrm{w}^{\prime} \\
\mathrm{K}^{\prime} ; \mathrm{w}^{\prime} \stackrel{\Delta}{\hookrightarrow} K^{\prime} ; e^{\prime} \\
\text { validConf }\left(\mathrm{K}^{\prime} ; \mathrm{w}^{\prime}\right)
\end{array}
$$

We prove (d) by 6.19, 6.23 and 6.33 ; (e) by 6.31 ; and (f) by 6.32 .

- Case $K ; e_{0}$ is a non-terminal configuration, so there exists $e_{0}^{\prime}$ s.t.:

$$
K ; e_{0} \longrightarrow K^{\prime} ; e_{0}^{\prime}
$$

By Rule RC-ECTX using 6.33:

$$
K ; E_{0}\left[e_{0}\right] \longrightarrow K^{\prime} ; E_{0}\left[e_{0}^{\prime}\right]
$$

By Lemma 12 using 6.14 and 6.33:

$$
\begin{array}{r}
\mathrm{S} ; \mathrm{L} ; \cdot ; \mathrm{H} ; \mathrm{B}_{0} \longrightarrow{ }^{*} \mathrm{~K}^{\prime} ; \mathrm{w}^{\prime} \\
\mathrm{K}^{\prime} ; \mathrm{w}^{\prime} \stackrel{\Delta}{\hookrightarrow} K^{\prime} ; e_{0}^{\prime}
\end{array}
$$

And we examine cases on the form of $B_{0}$ for the last result of the above lemma: 
- Case $B_{0} \equiv \mathrm{E}[1 . \mathrm{m}(\overline{\mathrm{v}})]$. It holds that:

$$
\mathrm{K}^{\prime} ; \mathrm{w}^{\prime} \equiv \mathrm{S} ; \mathrm{L}_{1} ;(\mathrm{L}, \mathrm{E}) ; \mathrm{H} ; \mathrm{B}_{1}
$$

So 6.36 becomes:

$$
\mathrm{S} ; \mathrm{L}_{1} ;(\mathrm{L}, \mathrm{E}) ; \mathrm{H} ; \mathrm{B} 1 \stackrel{\Delta}{\hookrightarrow} K^{\prime} ; e_{0}^{\prime}
$$

By inverting S-EXP-RTCONF on 6.38:

$$
\mathrm{L}_{1} ;(\mathrm{L}, \mathrm{E}) ; \mathrm{B}_{1} \stackrel{\mathrm{H}, \Delta}{\longrightarrow} e_{0}^{\prime}
$$

By Lemma 7 using 6.13 and 6.39:

$$
\mathrm{L}_{1} ;\left(\mathrm{X}_{0} ; \mathrm{L}_{0}, \mathrm{E}_{0} ; \mathrm{L}, \mathrm{E}\right) ; \mathrm{B}_{1} \stackrel{\mathrm{H}, \Delta}{\longrightarrow} E_{0}\left[e_{0}^{\prime}\right]
$$

Let:

$$
\mathrm{X}^{\prime} \equiv \mathrm{X}_{0} ; \mathrm{L}_{0}, \mathrm{E}_{0} ; \mathrm{L}, \mathrm{E}
$$

By applying Rule S-EXP-RTCONF on 6.2, 6.3 and 6.40:

$$
\mathrm{S} ; \mathrm{L}_{1} ; \mathrm{X}^{\prime} ; \mathrm{H} ; \mathrm{B}_{1} \stackrel{\Delta}{\hookrightarrow} K^{\prime} ; E_{0}\left[e_{0}^{\prime}\right]
$$

By Lemma 11 on 6.35:

$$
\mathrm{S} ; \mathrm{L} ; \mathrm{X} ; \mathrm{H} ; \mathrm{B}_{0} \longrightarrow^{*} \mathrm{~S} ; \mathrm{L}_{1} ; \mathrm{X}^{\prime} ; \mathrm{H} ; \mathrm{B}_{1}
$$

We prove (d), (e) and (f) by $6.43,6.42$ and 6.37 , respectively.

- For all remaining cases on $\mathrm{B}_{0}$ :

$$
\begin{aligned}
& \mathrm{H}^{\prime} \supseteq \mathrm{H} \\
& \mathrm{L}^{\prime} \supseteq \mathrm{L}
\end{aligned}
$$

Because of 6.11 , it holds that:

$$
\mathrm{K}^{\prime} ; \mathrm{w}^{\prime} \equiv \mathrm{S} ; \mathrm{L}^{\prime} ; \cdot ; \mathrm{H}^{\prime} ; \mathrm{B}^{\prime}
$$

By inverting Rule S-EXP-RTCONF on 6.36:

$$
\mathrm{K}^{\prime} ; \mathrm{H}^{\prime} \hookrightarrow H^{\prime}
$$

By Lemma 11 on 6.35:

$$
\mathrm{S} ; \mathrm{L} ; \mathrm{X} ; \mathrm{H} ; \mathrm{B}_{0} \longrightarrow^{*} \mathrm{~S} ; \mathrm{L}^{\prime} ; \mathrm{X} ; \mathrm{H}^{\prime} ; \mathrm{B}^{\prime}
$$

Fact 6.36 becomes:

$$
\mathrm{S} ; \mathrm{L}^{\prime} ; \cdot ; \mathrm{H}^{\prime} ; \mathrm{B}^{\prime} \stackrel{\Delta}{\hookrightarrow} K^{\prime} ; e_{0}^{\prime}
$$

By inverting S-EXP-RTCONF on 6.49:

$$
\mathrm{L}^{\prime} ; \cdot ; \mathrm{B}^{\prime} \stackrel{\mathrm{H}^{\prime}, \Delta}{\longrightarrow} e_{0}^{\prime}
$$

By applying Lemma 6 on 6.13 using 6.44:

$$
\mathrm{L}_{0} ; \mathrm{X}_{0} ; \mathrm{E}_{0} \stackrel{\mathrm{H}^{\prime}, \Delta}{\longrightarrow} E_{0}
$$

By applying rule S-STACK-CONS on 6.13 and 6.50:

$$
\mathrm{L}^{\prime} ;\left(\mathrm{X}_{0} ; \mathrm{L}_{0}, \mathrm{E}_{0}\right) ; \mathrm{B}^{\prime} \stackrel{\mathrm{H}^{\prime}, \Delta}{\longrightarrow} E_{0}\left[e_{0}^{\prime}\right]
$$

By applying rule S-EXP-RTCONF on 6.2, 6.47 and 6.52:

$$
\mathrm{S} ; \mathrm{L}^{\prime} ; \mathrm{X} ; \mathrm{H}^{\prime} ; \mathrm{B}^{\prime} \stackrel{\Delta}{\hookrightarrow} K^{\prime} ; E_{0}\left[e_{0}^{\prime}\right]
$$

We prove (d), (e) and (f) by 6.48, 6.53 and 6.46, respectively. 
Theorem 2 (Forward Simulation). If $\mathrm{R} \stackrel{\Delta}{\hookrightarrow} R$, then:

(a) if $R$ is terminal, then there exists $\mathrm{R}^{\prime}$ s.t. $\mathrm{R} \longrightarrow \mathrm{R}^{\prime}$ and $\mathrm{R}^{\prime} \stackrel{\Delta}{\hookrightarrow} R$

(b) if $R \longrightarrow R^{\prime}$, then there exists $\mathrm{R}^{\prime}$ s.t. $\mathrm{R} \longrightarrow \mathrm{R}^{\prime}$ and $\mathrm{R}^{\prime} \stackrel{\Delta}{\hookrightarrow} R^{\prime}$

Proof. Part (a) is proven by use of by Lemma 10, and part (b) by Lemma 13. 


\section{B.2 Type Safety}

Lemma 14 (Substitution Lemma). If

(a) $\Gamma \vdash \bar{w}: \bar{S}$

(b) $\Gamma, \bar{x}: \bar{S} \vdash \bar{S} \leq \bar{S}^{\prime}$

(c) $\Gamma, \bar{x}: \bar{S}^{\prime} \vdash e: T$

then

$\Gamma \vdash[\bar{w} / \bar{x}] e: R, R \leq T$

Proof. By induction on the derivation of the statement $\Gamma, \bar{x}: \bar{S} \vdash e: T$.

Lemma 15 (Environment Substitution). If $\Gamma_{1}, x: T, \Gamma_{2} \vdash w: S$, then $\Gamma_{1}, x: T,[z / x] \Gamma_{2} \vdash[z / x] w:[z / x] S$.

Proof. Straightforward.

Lemma 16 (Weakening Subtyping). If $\Gamma \vdash S \leq T$, then $\Gamma, x: R \vdash S \leq T$.

Proof. Straightforward.

Lemma 17 (Weakening Typing). If $\Gamma \vdash e: T$, then for $\Gamma^{\prime} \supseteq \Gamma$, it holds that $\Gamma^{\prime} \vdash e: T$.

Proof. Straightforward.

Lemma 18 (Store Type). If $\Sigma \vdash H, H(l)=O$ and $\Sigma(l)=T$, then $\Sigma \vdash_{H} O: S, T \leq S$.

Proof. Straightforward.

Lemma 19 (Method Body Type - Lemma A.3 from [25]). If

(a) $\Gamma, z: T \vdash z$ has $(\operatorname{def} m(\bar{z}: \bar{R})\{p\}: S=e)$

(b) $\Gamma, z: T, \bar{z}: \bar{T} \vdash \bar{T} \leq \bar{R}$

Then for some type $S^{\prime}$ it is the case that:

$\Gamma, z: T, \bar{z}: \bar{T} \vdash e: S^{\prime}, S^{\prime} \leq S$

Proof. Straightforward.

Lemma 20 (Cast). If $\Sigma \vdash H$ and $\Gamma ; \Sigma \vdash l: S, S \lesssim T$, then $\Gamma ; \Sigma \vdash H(l): R, R \leq T$.

Proof. Straightforward.

Lemma 21 (Evaluation Context Typing). If $\Gamma \vdash E[e]: T$, then for some type $S$ it holds that $\Gamma \vdash e: S$.

Proof. By induction on the structure of the evaluation context $E$.

Lemma 22 (Evaluation Context Step Typing). If

$\Gamma ; \Sigma \vdash E[e]: T, e: S$

and for some expression $e^{\prime}$ and heap typing $\Sigma^{\prime} \supseteq \Sigma$ it holds that

$\Gamma ; \Sigma^{\prime} \vdash e^{\prime}: S^{\prime}, S^{\prime} \lesssim S$

then

$\Gamma ; \Sigma^{\prime} \vdash E\left[e^{\prime}\right]: T^{\prime}, T^{\prime} \lesssim T$

Proof. By induction on the structure of the evaluation context $E$.

Lemma 23 (Selfification). If $\Gamma, x: S \vdash S \leq T$ then $\Gamma, x: S \vdash S \leq \operatorname{self}(T, x)$.

Proof. Straightforward.

Lemma 24 (Existential Weakening). If $\Gamma \vdash R \leq R^{\prime}$ then $\Gamma \vdash \exists x: R . T \leq \exists x: R^{\prime} . T$. 
Proof. Straightforward.

Lemma 25 (Boolean Facts). If

(a) $\Gamma \vdash x: T, T \leq\{\nu:$ bool $\mid \nu=$ true $\}$

(b) $\Gamma, x \vdash e: S, S \leq T$

then

$$
\Gamma \vdash e: S, S \leq T
$$

Proof. Straightforward.

Theorem 3 (Subject Reduction). If

(a) $\Gamma ; \Sigma \vdash e: T$

(b) $K ; e \longrightarrow K^{\prime} ; e^{\prime}$

(c) $\Sigma \vdash K . H$

then for some $T^{\prime}$ and $\Sigma^{\prime} \supseteq \Sigma$ :

(d) $\Gamma ; \Sigma^{\prime} \vdash e^{\prime}: T^{\prime}$

(e) $\Gamma \vdash T^{\prime} \lesssim T$

(f) $\Sigma^{\prime} \vdash H^{\prime}$.

Proof. We proceed by induction on the structure of fact (b):

$$
K ; e \longrightarrow K^{\prime} ; e^{\prime}
$$

We have the following cases:

- [RC-ECTX]: Fact (b) has the form:

$$
K ; E\left[e_{0}\right] \longrightarrow K^{\prime} ; E\left[e_{0}^{\prime}\right]
$$

From (a):

$$
\Gamma ; \Sigma \vdash E\left[e_{0}\right]: T
$$

By Lemma 21 on 6.2:

$$
\Gamma ; \Sigma \vdash e_{0}: T_{0}
$$

By inverting Rule RC-ECTX on 6.1:

$$
K ; e_{0} \longrightarrow K^{\prime} ; e_{0}^{\prime}
$$

By induction hypothesis, using 6.3, 6.4 and (c) we get:

$$
\begin{array}{r}
\Gamma ; \Sigma^{\prime} \vdash e_{0}^{\prime}: T_{0}^{\prime} \\
\Gamma ; \Sigma^{\prime} \vdash T_{0}^{\prime} \lesssim T_{0} \\
\Sigma^{\prime} \vdash K^{\prime} . H \\
\Sigma^{\prime} \supseteq \Sigma
\end{array}
$$

For some type $T_{0}^{\prime}$ and heap $K^{\prime} . H$.

From 6.7 we prove (f).

By Lemma 22 using 6.2, 6.3, 6.5, 6.6 and 6.8:

$$
\Gamma ; \Sigma^{\prime} \vdash E\left[e_{0}^{\prime}\right]: T^{\prime}, T^{\prime} \lesssim T
$$

From 6.9 we prove (d) and (e). 
- [R-FIELD]: Fact (b) has the form:

$$
K ; l . h \longrightarrow K ; v
$$

By Fact (a) for $e \equiv l . h$ we have:

$$
\Gamma ; \Sigma \vdash l . h: T
$$

By inverting R-FIELD on 6.10:

$$
\begin{array}{r}
K . H(l) \equiv O=\left\{\text { proto: } l^{\prime} ; \mathrm{f}: \widetilde{F}\right\} \\
f:=v \in \widetilde{F}
\end{array}
$$

By inverting WF-HEAP-INST on (c) for location $l$ :

$$
\begin{array}{r}
\widetilde{F} \doteq \circ \bar{f}:=\bar{v}_{\mathrm{I}}, \square \bar{g}:=\bar{v}_{\mathrm{M}} \\
\lfloor\Sigma(l)\rfloor=C \\
\Gamma, z: C \vdash \text { fields }(z)=\circ \bar{f}: \bar{R}, \square \bar{g}: \bar{U} \\
\Sigma \vdash \bar{v}_{\mathrm{I}}: \bar{T}_{\mathrm{I}} \\
\Sigma \vdash \bar{v}_{\mathrm{M}}: \bar{T}_{\mathrm{M}} \\
\Gamma, z: C, \bar{z}_{\mathrm{I}}: \operatorname{self}\left(\bar{T}_{\mathrm{I}}, z \cdot \bar{f}\right) \vdash \bar{T}_{\mathrm{I}} \leq \bar{R}, \bar{T}_{\mathrm{M}} \leq \bar{U}, \operatorname{inv}(C, z)
\end{array}
$$

By applying RT-T-OBJ on $6.15,6.14$ and 6.17 :

$$
\Gamma ; \Sigma \vdash O: S^{\prime}
$$

Where:

$$
S^{\prime} \equiv \exists \bar{z}_{\mathrm{I}}: \bar{T}_{\mathrm{I}} \cdot\left\{\nu: C \mid \nu \cdot \bar{f}=\bar{z}_{\mathrm{I}} \wedge \operatorname{inv}(C, \nu)\right\}
$$

By Lemma 18 using (c), 6.12 and 6.15:

$$
\Gamma \vdash S \leq S^{\prime}
$$

Where:

$$
\Sigma(l)=S
$$

We examine cases on the typing statement 6.11 :

- [T-FIELD-I]: Field $h$ is an immutable field $f_{i}$, so fact 6.11 becomes:

$$
\Gamma ; \Sigma \vdash l . f_{i}: \exists z: S . \text { self }\left(R_{i}, z . f_{i}\right)
$$

By inverting T-FIELD-I on 6.24:

$$
\begin{array}{r}
\Sigma \vdash l: S \\
\Gamma, z: S ; \Sigma \vdash z \text { haslmm } f_{i}: R_{i}
\end{array}
$$

For a fresh $z$.

Keeping only the relevant part of 6.17 and 6.19:

$$
\begin{array}{r}
\Gamma ; \Sigma \vdash v_{i}: T_{i} \\
\Gamma, z: C, \bar{z}_{\mathrm{I}}: \operatorname{self}\left(\bar{T}_{\mathrm{I}}, z \cdot \bar{f}\right) ; \Sigma \vdash T_{i} \leq R_{i}
\end{array}
$$


By 6.27 we prove (d).

By Lemma 23 using 6.28 and picking $z_{i}$ as the selfification variable:

$$
\Gamma, z: C, \bar{z}_{\mathrm{I}}: \operatorname{self}\left(\bar{T}_{\mathrm{I}}, z \cdot \bar{f}\right) ; \Sigma \vdash T_{i} \leq \operatorname{self}\left(R_{i}, z_{i}\right)
$$

For the above environment it holds that:

$$
\llbracket \Gamma, z: C, \bar{z}_{\mathrm{I}}: \operatorname{self}\left(\bar{T}_{\mathrm{I}}, z \cdot \bar{f}\right) ; \Sigma \rrbracket \Rightarrow z_{i}=z \cdot f_{i}
$$

By $\leq$-REFL and By Lemma 23 using 6.30:

$$
\Gamma, z: C, \bar{z}_{\mathrm{I}}: \operatorname{self}\left(\bar{T}_{\mathrm{I}}, z \cdot \bar{f}\right) ; \Sigma \vdash \operatorname{self}\left(R_{i}, z_{i}\right) \leq \operatorname{self}\left(\operatorname{self}\left(R_{i}, z_{i}\right), z \cdot f_{i}\right)
$$

By simplifying 6.31 using $\leq$-TRANS on 6.29 and 6.31 we get:

$$
\Gamma, z: C, \bar{z}_{\mathrm{I}}: \operatorname{self}\left(\bar{T}_{\mathrm{I}}, z \cdot \bar{f}\right) ; \Sigma \vdash T_{i} \leq \operatorname{self}\left(R_{i}, z \cdot f_{i}\right)
$$

By 6.32 it also holds that:

$$
\Gamma, z: \exists \bar{z}_{\mathrm{I}}: \operatorname{self}\left(\bar{T}_{\mathrm{I}}, z \cdot \bar{f}\right) \cdot C \vdash T_{i} \leq \operatorname{self}\left(R_{i}, z \cdot f_{i}\right)
$$

By 6.33 it also holds that:

$$
\Gamma, z: \exists \bar{z}_{\mathrm{I}}: \bar{T}_{\mathrm{I}} \cdot \operatorname{self}\left(C, \bar{z}_{\mathrm{I}}\right) \vdash T_{i} \leq \operatorname{self}\left(R_{i}, z \cdot f_{i}\right)
$$

By expanding 6.34 and 6.19:

$$
\Gamma, z: \exists \bar{z}_{\mathrm{I}}: \bar{T}_{\mathrm{I}} \cdot\left\{\nu: C \mid \nu \cdot \bar{f}=\bar{z}_{\mathrm{I}} \wedge \operatorname{inv}(C, \nu)\right\} \vdash T_{i} \leq \operatorname{self}\left(R_{i}, z \cdot f_{i}\right)
$$

By using 6.21 on 6.35 :

$$
\Gamma, z: S^{\prime} \vdash T_{i} \leq \operatorname{self}\left(R_{i}, z \cdot f_{i}\right)
$$

By Lemma 16 using 6.36 and 6.22:

$$
\Gamma, z: S \vdash T_{i} \leq \operatorname{self}\left(R_{i}, z \cdot f_{i}\right)
$$

From Rule $\leq$-WiTNESS using 6.45:

$$
\Gamma \vdash T_{i} \leq \exists z: S . \operatorname{self}\left(R_{i}, z . f_{i}\right)
$$

Using 6.24, 6.17 and 6.38 we prove (e).

Heap $K . H$ does not evolve so (f) holds trivially.

- [T-FIELD-M]: Field $h$ is a mutable field $g_{i}$, so fact (a) becomes:

$$
\Gamma ; \Sigma \vdash l . g_{i}: \exists z: S . V_{i}
$$

By inverting T-FIELD-M on 6.39:

$$
\begin{array}{r}
\Gamma \vdash l: S \\
\Gamma, l: S \vdash z \text { hasMut } g_{i}: U_{i}
\end{array}
$$

For a fresh $z$.

Keeping only the relevant parts of 6.17 and 6.19:

$$
\begin{array}{r}
\Gamma \vdash v_{i}: T_{i} \\
\Gamma, z: C, \bar{z}_{\mathrm{I}}: \operatorname{self}\left(\bar{T}_{\mathrm{I}}, z \cdot \bar{f}\right) \vdash T_{i} \leq U_{i}
\end{array}
$$


By 6.42 we prove (d).

By similar reasoning as before and using 6.43 we get:

$$
\Gamma, z: S^{\prime} \vdash T_{i} \leq U_{i}
$$

By Lemma 16 using 6.44 and 6.22:

$$
\Gamma, z: S \vdash T_{i} \leq U_{i}
$$

By Rule $\leq$-WitNESS using 6.45:

$$
\Gamma \vdash T_{i} \leq \exists z: S . U_{i}
$$

Using 6.39, 6.17 and 6.46 we prove (e).

Heap $K . H$ does not evolve so (f) holds trivially.

- [R-CALL]: Fact (b) has the form:

$$
K ; l . m(\bar{v}) \longrightarrow K ;[\bar{v} / \bar{z}, l / \text { this }] e^{\prime}
$$

By (a) for $e \equiv l . m(\bar{v})$ we have:

$$
\Gamma ; \Sigma \vdash \operatorname{l.m}(\bar{v}): \exists z: T . \exists \bar{z}: \bar{T} . S
$$

By inverting T-INV on 6.48:

$$
\begin{array}{r}
\Gamma ; \Sigma \vdash l: T, \bar{v}: \bar{T} \\
\Gamma, z: T, \bar{z}: \bar{T} \vdash z \text { has }\left(\operatorname{def} m(\bar{z}: \bar{R})\{p\}: S=e^{\prime}\right) \\
\Gamma, z: T, \bar{z}: \bar{T} \vdash \bar{T} \leq \bar{R} \\
\Gamma, z: T, \bar{z}: \bar{T} \vdash p
\end{array}
$$

With fresh $z$ and $\bar{z}$.

By inverting R-CALL on 6.47:

$$
\begin{array}{r}
\text { resolveMethod }(H, l, m)=(\operatorname{def} m(\bar{z}: \bar{R})\{p\}: S=e) \\
\text { eval }(p)=\text { true }
\end{array}
$$

Note that this has already been substituted by $l$ in $S$ and $p$.

By Lemma 19 using 6.50 and 6.51:

$$
\Gamma, z: T, \bar{z}: \bar{T} \vdash e^{\prime}: S^{\prime}, S^{\prime} \leq S
$$

By 6.55 we prove (d).

By Rule $\leq$-WitnESS using 6.55:

$$
\Gamma \vdash S^{\prime} \leq \exists z: T . \exists \bar{z}: \bar{T} . S
$$

By Lemma 14 using 6.49, 6.51 and 6.55:

$$
\Gamma \vdash[\bar{v} / \bar{z}, l / \text { this }] e^{\prime}: U, U \leq S^{\prime}
$$

By Rule $\leq-$ TRANS on 6.55 and 6.57 :

$$
\Gamma \vdash U \leq \exists z: T . \exists \bar{z}: \bar{T} . S
$$

By 6.58 we prove (e).

Heap $K . H$ does not evolve so (f) holds trivially. 
- [R-CAST]: Fact (b) has the form:

$$
K ; l \text { as } T \longrightarrow K ; l
$$

By (a) for $e \equiv l$ as $T$ we have:

$$
\Gamma ; \Sigma \vdash l \text { as } T: T
$$

By inverting T-CAST on 6.59:

$$
\begin{array}{r}
\Gamma ; \Sigma \vdash l: S \\
\Gamma \vdash T \\
\Gamma \vdash S \lesssim T
\end{array}
$$

By 6.60 and 6.62 we get (d) and (e), respectively.

$K . H$ does not evolve, which proves (f), given (b).

- [R-NEw]: Fact (c) has the form:

$$
K ; \text { new } C(\bar{v}) \longrightarrow K^{\prime} ; l
$$

By inverting R-NEW on 6.63:

$$
\begin{array}{r}
\mathrm{H}\left(l_{0}\right)=\left\{\text { name: } C \text {; proto: } l_{0}^{\prime} ; \mathrm{m}: \widetilde{M}\right\} \\
\text { fields }(S, C)=\bar{f}: \bar{T} \\
O=\left\{\text { proto: } l_{0} ; \mathrm{f}: \bar{f}:=\bar{v}\right\} \\
H^{\prime}=H[l \mapsto O]
\end{array}
$$

By (a) for $e \equiv$ new $C(\bar{v})$ we have:

$$
\Gamma ; \Sigma \vdash \text { new } C(\bar{v}): R_{0}
$$

Where:

$$
R_{0} \equiv \exists \bar{z}_{\mathrm{I}}: \bar{T}_{\mathrm{I}} \cdot\left\{\nu: C \mid \nu \cdot \bar{f}=\bar{z}_{\mathrm{I}} \wedge \operatorname{inv}(C, \nu)\right\}
$$

By inverting T-New on 6.68:

$$
\begin{aligned}
& \Gamma \vdash \bar{v}:\left(\bar{T}_{\mathrm{I}}, \bar{T}_{\mathrm{M}}\right) \\
& \vdash \text { class }(C) \\
& \Gamma, z: C \vdash \text { fields }(z)=\circ \bar{f}: \bar{R}, \square \bar{g}: \bar{U} \\
& \Gamma, z: C, \bar{z}: \bar{T}, z \cdot \bar{f}=\bar{z}_{\mathrm{I}} \vdash \bar{T}_{\mathrm{I}} \leq \bar{R}, \bar{T}_{\mathrm{M}} \leq \bar{U}, \operatorname{inv}(C, z)
\end{aligned}
$$

For fresh $z$ and $\bar{z}$.

We choose a heap typing $\Sigma^{\prime}$, such that:

$$
\Sigma^{\prime}=\Sigma\left[l \mapsto R_{0}\right]
$$

Hence:

$$
\Sigma^{\prime}(l)=R_{0}
$$

By applying Rule RT-T-LoC using 6.74:

$$
\Gamma ; \Sigma^{\prime} \vdash l: R_{0}
$$

Which proves (d). 
By applying Rule RT-T-OBJ using 6.74, 6.66 and 6.70:

$$
K \vdash_{\Sigma} O: R_{0}
$$

By $\leq$-ID we trivially get:

$$
\Gamma \vdash R_{0} \leq R_{0}
$$

Which proves (e).

By applying Rule WF-HEAP-INST on 6.66, 6.64, 6.74, 6.72, 6.70 and 6.73:

$$
\Sigma^{\prime} \vdash K^{\prime} . H
$$

Which proves (f).

- [R-LETIN] Similar approach to case R-CALL.

- [R-DotAsGN]: Fact (b) has the form:

$$
K ; l . g_{i} \leftarrow v^{\prime} \longrightarrow K^{\prime} ; v^{\prime}
$$

By inverting Rule R-DotAsGN on 6.77:

$$
H^{\prime}=K . H\left[l \mapsto K . H(l)\left[g_{i} \mapsto v^{\prime}\right]\right]
$$

From (a) for $e \equiv l . g_{i} \leftarrow v^{\prime}$ :

$$
\Gamma ; \Sigma \vdash l . g_{i} \leftarrow v^{\prime}: T^{\prime}
$$

By inverting Rule T-ASGN on 6.79:

$$
\begin{array}{r}
\Gamma ; \Sigma \vdash l: T_{l}, v^{\prime}: T^{\prime} \\
\Gamma, z:\left\lfloor T_{l}\right\rfloor ; \Sigma \vdash z \text { hasMut } g_{i}: U_{i}, T^{\prime} \leq U_{i}
\end{array}
$$

For a fresh $z$.

By 6.80 and $\leq$-REFL we prove (d) and (e).

By inverting RT-T-LOC on 6.80:

$$
\Sigma(l)=T_{l}
$$

By inverting WF-HEAP-INST on (c) for location $l$ and using 6.82:

$$
\begin{array}{r}
\left.O \doteq \text { \{proto: } l^{\prime} ; \mathrm{f}: \widetilde{F}\right\} \\
\widetilde{F} \doteq \circ \bar{f}:=\bar{v}_{\mathrm{I}}, \square \bar{g}:=\bar{v}_{\mathrm{M}} \\
\lfloor\Sigma(l)\rfloor=C \\
\Gamma, z: C \vdash \text { fields }(z)=\circ \bar{f}: \bar{R}, \square \bar{g}: \bar{U} \\
\Sigma \vdash \bar{v}_{\mathrm{I}}: \bar{T}_{\mathrm{I}} \\
\Sigma \vdash \bar{v}_{\mathrm{M}}: \bar{T}_{\mathrm{M}} \\
\Gamma, z: C, \bar{z}_{\mathrm{I}}: \operatorname{self}\left(\bar{T}_{\mathrm{I}}, z \cdot \bar{f}\right) \vdash \bar{T}_{\mathrm{I}} \leq \bar{R}, \bar{T}_{\mathrm{M}} \leq \bar{U}, \operatorname{inv}(C, z)
\end{array}
$$

Fact 6.78 becomes:

$$
\begin{aligned}
H^{\prime} & =K . H\left[l \mapsto O^{\prime}\right] \\
O^{\prime} & =\left\{\text { proto: } l^{\prime} ; \mathrm{f}: \widetilde{F}^{\prime}\right\} \\
\widetilde{F}^{\prime} & =\circ \bar{f}:=\bar{v}_{\mathrm{I}}, \square \bar{g}:=\bar{v}_{\mathrm{M}}^{\prime} \\
\bar{v}_{\mathrm{M}}^{\prime} & =\bar{v}_{\mathrm{M}, . . i-1}, v_{\mathrm{M}, i}^{\prime}, \bar{v}_{\mathrm{M}, i+1 . .}
\end{aligned}
$$


Also by 6.80 and 6.88 it holds that:

$$
\Sigma \vdash \bar{v}_{\mathrm{M}}^{\prime}:\left(\bar{T}_{\mathrm{M}, . . i-1}, T^{\prime}, \bar{T}_{\mathrm{M}, i+1 . .}\right)
$$

By Lemma 16 on 6.81:

$$
\Gamma, z: C, \bar{z}_{\mathrm{I}}: \operatorname{self}\left(\bar{T}_{\mathrm{I}}, z \cdot \bar{f}\right) ; \Sigma \vdash T^{\prime} \leq U_{i}
$$

By applying Rule WF-HEAP-INST on 6.91, 6.92, 6.85, 6.86, 6.87, 6.94, 6.89 and 6.95:

$$
\Sigma \vdash H^{\prime}
$$

Which proves (f).

- [R-LETIF]: Assume $c \equiv$ true (the case for false is symmetric).

Fact (b) has the form:

$$
K \text {; letif }\left[\bar{x}, \bar{x}_{1}, \bar{x}_{2}\right] \text { (true) } ? u_{1}: u_{2} \text { in } e \longrightarrow K ; u_{1}\left\langle\left[\bar{x}_{1} / \bar{x}\right] e\right\rangle
$$

By Rule T-CTX fact (a) has the form:

$$
\Gamma \vdash \operatorname{letif}\left[\bar{x}, \bar{x}_{1}, \bar{x}_{2}\right] \text { (true) } ? u_{1}: u_{2} \text { in } e: \exists \bar{x}: \bar{S} . R
$$

So type $T$ has the form:

$$
T \equiv \exists \bar{x}: \bar{S} \cdot R
$$

By inverting Rule T-CTX on (a):

$$
\begin{array}{r}
\Gamma \vdash \operatorname{letif}\left[\bar{x}, \bar{x}_{1}, \bar{x}_{2}\right]\left(\text { true) } ? u_{1}: u_{2} \text { in }\langle\rangle \triangleright \bar{x}: \bar{S}\right. \\
\Gamma, \bar{x}: \bar{S} \vdash e: R
\end{array}
$$

By inverting Ryle T-LETIF on 6.99:

$$
\begin{array}{r}
\Gamma \vdash \text { true }: S, S \leq \text { bool } \\
\Gamma, z: S, z \vdash u_{1} \triangleright \Gamma_{1} \\
\Gamma, z: S, \neg z \vdash u_{2} \triangleright \Gamma_{2} \\
\Gamma, \Gamma_{1} \vdash \Gamma_{1}\left(\bar{x}_{1}\right) \leq \bar{S} \\
\Gamma, \Gamma_{2} \vdash \Gamma_{2}\left(\bar{x}_{2}\right) \leq \bar{S} \\
\Gamma \vdash \bar{S}
\end{array}
$$

By Rule T-CsT on true:

$$
\Gamma \vdash \text { true }:\{\nu: \text { bool } \mid \nu=\text { true }\}
$$

By Lemma 25 on 6.101 and 6.102:

$$
\Gamma \vdash u_{1} \triangleright \Gamma_{1}
$$

Environment $\Gamma_{1}$ has the form:

$$
\Gamma_{1} \equiv \bar{x}_{1}: \Gamma_{1}\left(\bar{x}_{1}\right), \bar{x}_{1}^{\prime}: \Gamma_{1}\left(\bar{x}_{1}^{\prime}\right)
$$

For some $\bar{x}_{1}^{\prime}$.

By Lemma 15 using 6.100:

$$
\Gamma, \bar{x}_{1}: \bar{S} \vdash\left[\bar{x}_{1} / \bar{x}\right] e:\left[\bar{x}_{1} / \bar{x}\right] R
$$


By Lemma 17 using 6.110:

$$
\Gamma, \bar{x}_{1}: \bar{S}, \bar{x}_{1}^{\prime}: \Gamma_{1}\left(\bar{x}_{1}^{\prime}\right) \vdash\left[\bar{x}_{1} / \bar{x}\right] e:\left[\bar{x}_{1} / \bar{x}\right] R
$$

By applying rule T-CTX on 6.108 and 6.111:

$$
\Gamma \vdash u\left\langle\left[\bar{x}_{1} / \bar{x}\right] e\right\rangle: \exists \bar{x}_{1}: \Gamma_{1}\left(\bar{x}_{1}\right) . \exists \bar{x}_{1}^{\prime}: \Gamma_{1}\left(\bar{x}_{1}^{\prime}\right) \cdot\left[\bar{x}_{1} / \bar{x}\right] R
$$

Which proves (d).

Fact 6.112 can be rewritten as:

$$
\Gamma \vdash u\left\langle\left[\bar{x}_{1} / \bar{x}\right] e\right\rangle: \exists \bar{x}: \Gamma_{1}(\bar{x}) . \exists \bar{x}_{1}^{\prime}: \Gamma_{1}\left(\bar{x}_{1}^{\prime}\right) . R
$$

Applying Rule $\leq$-BIND using 6.113:

$$
\Gamma \vdash \exists \bar{x}: \Gamma_{1}(\bar{x}) . \exists \bar{x}_{1}^{\prime}: \Gamma_{1}\left(\bar{x}_{1}^{\prime}\right) . R \leq \exists \bar{x}: \Gamma_{1}(\bar{x}) . R
$$

By Lemma 24 on the right-hand side of 6.114 :

$$
\Gamma \vdash \exists \bar{x}: \Gamma_{1}(\bar{x}) . R \leq \exists \bar{x}: S . R
$$

By 6.113, 6.114 and 6.115, and using Rule $\leq$-TRANS we prove (e).

Heap $K . H$ does not evolve so (f) holds trivially.

Theorem 4 (Progress). If

(a) $\Gamma ; \Sigma \vdash e: T$,

(b) $\Sigma \vdash H$

then one of the following holds:

- $e$ is a value,

- there exist $e^{\prime}, H^{\prime}$ and $\Sigma^{\prime} \supseteq \Sigma$ s.t. $\Sigma^{\prime} \vdash H^{\prime}$ and $H ; e \longrightarrow H^{\prime} ; e^{\prime}$.

Proof. We proceed by induction on the structure of derivation (a):

$\cdot[$ T-FIELD-I]

$$
\Gamma ; \Sigma \vdash e_{0} \cdot f_{i}: \exists z: T_{0} . \operatorname{self}\left(T, z \cdot f_{i}\right)
$$

By inverting T-FIELD-I on 2.1:

$$
\begin{array}{r}
\Gamma ; \Sigma \vdash e_{0}: T_{0} \\
\Gamma, z: T_{0} ; \Sigma \vdash z \text { haslmm } f_{i}: T
\end{array}
$$

By i.h. using 2.2 and (b) there are two possible cases on $e_{0}$ :

- $\left[e_{0} \equiv l_{0}\right]$ Statement 2.2 becomes:

$$
\Gamma ; \Sigma \vdash l_{0}: T_{0}
$$

By (b) for location $l_{0}$ :

$$
\Sigma \vdash H\left[l_{0} \mapsto O\right]
$$

Where:

$$
O \equiv\left\{\text { proto: } l_{0}^{\prime} ; \mathrm{f}: \widetilde{F}\right\}
$$


By Lemma 18 using (b) and 2.5:

$$
\begin{aligned}
\Sigma\left(l_{0}\right) & =T_{0} \\
\Gamma ; \Sigma \vdash O: S_{0}, S_{0} & \leq T_{0}
\end{aligned}
$$

By Lemma A.6 in [25] using 2.3 and 2.8:

$$
\Gamma, z: S_{0} ; \Sigma \vdash z \text { haslmm } f_{i}: T
$$

By applying Rule R-FIELD using 2.5, 2.6 and 2.9:

$$
H ; l_{0} . f_{i} \longrightarrow H ; v_{i}
$$

- $\left[\exists e_{0}^{\prime}\right.$ s.t. $\left.H ; e_{0} \longrightarrow H^{\prime} ; e_{0}^{\prime}\right]$ By applying Rule RC-ECTX:

$$
H ; e_{0} . f_{i} \longrightarrow H^{\prime} ; e_{0}^{\prime} \cdot f_{i}
$$

- [T-FIELD-M] Similar to previous case.

- [T-INV], [T-NEw] Similar to the respective case of CFJ [25].

$\cdot[\mathrm{T}-\mathrm{CAST}]:$

$$
\Gamma ; \Sigma \vdash e_{0} \text { as } T: T
$$

By inverting T-CAST on 2.10:

$$
\begin{array}{r}
\Gamma \vdash e_{0}: S_{0} \\
\Gamma ; \Sigma \vdash T \\
\Gamma ; \Sigma \vdash S_{0} \lesssim T
\end{array}
$$

By i.h. using 2.11 and (b) there are two possible cases on $e_{0}$ :

- $\left[e_{0} \equiv l_{0}\right]$ Statement 2.11 becomes:

$$
\Gamma ; \Sigma \vdash l_{0}: S_{0}
$$

By Lemma 20 using (b) and 2.13:

$$
\Gamma ; \Sigma \vdash H\left(l_{0}\right): R_{0}, R_{0} \leq T
$$

From R-CAST using 2.15:

$$
H ; l_{0} \text { as } T \longrightarrow H ; l_{0}
$$

- $\left[\exists e_{0}^{\prime}\right.$ s.t. $\left.H ; e_{0} \longrightarrow H^{\prime} ; e_{0}^{\prime}\right]$ By rule RC-ECTX:

$$
H ; e_{0} \text { as } T \longrightarrow H^{\prime} ; e_{0}^{\prime} \text { as } T
$$

- [T-LET], [T-ASGN], [T-IF] These cases are handled in a similar manner. 